\title{
Multimodel Operability Framework for Design of Modular and Intensified Energy Systems
}

Vitor Gazzaneo

West Virginia University, vigazzaneo@mix.wvu.edu

Follow this and additional works at: https://researchrepository.wvu.edu/etd

Part of the Other Chemical Engineering Commons, and the Process Control and Systems Commons

\section{Recommended Citation}

Gazzaneo, Vitor, "Multimodel Operability Framework for Design of Modular and Intensified Energy Systems" (2021). Graduate Theses, Dissertations, and Problem Reports. 8069.

https://researchrepository.wvu.edu/etd/8069

This Dissertation is protected by copyright and/or related rights. It has been brought to you by the The Research Repository @ WVU with permission from the rights-holder(s). You are free to use this Dissertation in any way that is permitted by the copyright and related rights legislation that applies to your use. For other uses you must obtain permission from the rights-holder(s) directly, unless additional rights are indicated by a Creative Commons license in the record and/ or on the work itself. This Dissertation has been accepted for inclusion in WVU Graduate Theses, Dissertations, and Problem Reports collection by an authorized administrator of The Research Repository @ WVU.

For more information, please contact researchrepository@mail.wvu.edu. 


\title{
MULTIMODEL OPERABILITY FRAMEWORK FOR DESIGN OF MODULAR AND INTENSIFIED ENERGY SYSTEMS
}

\author{
Vitor Gazzaneo
}

\author{
Dissertation submitted to the \\ Benjamin M. Statler College of Engineering and Mineral Resources \\ at West Virginia University \\ In partial fulfillment of the requirements for the degree of \\ Doctor of Philosophy in \\ Chemical Engineering
}

Fernando V. Lima, Ph.D., Chair

Debangsu Bhattacharyya, Ph.D.

YanFang Ye, Ph.D.

Stephen E. Zitney, Ph.D.

Thorsten Wuest, Ph.D.

David R. Vinson, Ph.D.

Department of Chemical and Biomedical Engineering

Morgantown, West Virginia

2021

Keywords: operability, process intensification, modular systems, surrogate modeling, high-dimensional nonlinear systems, natural gas utilization, membrane reactor, steam methane reforming, process operability app 


\title{
Abstract \\ Multimodel Operability Framework for Design of Modular and Intensified Energy Systems
}

\author{
Vitor Gazzaneo
}

In this dissertation, a novel operability framework is introduced for the process design of modular and intensified energy systems that are challenged by complexity and highly constrained environments. Previously developed process operability approaches are reviewed and further developed in terms of theory, application, and software infrastructure. An optimization-based multilayer operability framework is introduced for process design of nonlinear energy systems. In the first layer of this framework, a mixedinteger linear programming (MILP)-based iterative algorithm considers the minimization of footprint and achievement of process intensification targets. Then, in the second layer, an operability analysis is performed to incorporate key features of optimality and feasibility accounting for the system achievability and flexibility. The outcome of this framework consists of a set of modular designs, considering both the aspects of size and process operability. For this study and throughout this dissertation, the nonlinear system is represented by multiple linearized models, which results in lower computational expense and more efficient quantification of operability regions.

A systematic techno-economic analysis framework is also proposed for costing intensified modular systems. Conventional costing techniques are extended to allow estimation of capital and operating costs of modular units. Economy of learning concepts are included to consider the effect of experience curves on purchase costs. Profitability measures are scaled with respect to production of a chemical of interest for comparison with plants of traditional scale. Scenarios in which the modular technology presents break-even or further reduction in cost when compared to the traditional process are identified as a result. A framework for the development of process operability algorithms is provided as a software infrastructure outcome. Generated codes from the developed approaches are included in an open-source platform that will give researchers from 
academia and industry access to the algorithms. This platform has the purpose of dissemination and future improvement of process operability algorithms and methods.

To show versatility and efficacy of the developed approaches, a variety of applications are considered as follows: a membrane reactor for direct methane aromatization conversion to hydrogen and benzene (DMA-MR), the classical shower problem in process operability, a power plant cycling application for power generation with penetration of renewable energy sources, and a newly developed modular hydrogen unit. Applications to DMA-MR subsystems demonstrate employment of the multilayer framework to find a region with modular design candidates, which are then ranked according to an operability index. The most operable design is determined and contrasted with the optimal design with respect to process intensification in terms of footprint minimization, showing that optimality at fixed nominal operations does not necessarily ensure the best system operability. For the modular hydrogen unit application, the developed process operability framework provides guidelines for obtaining modular designs that are highly integrated and flexible with respect to disturbances in inlet natural gas composition. The modular hydrogen unit is also used for demonstration of the proposed techno-economic analysis framework. A comparison with a benchmark conventional steam methane reforming plant shows that the modular hydrogen unit can benefit from the economy of learning. An assembled modular steam methane reforming plant is used to map the decrease in natural gas price that must be needed for the plant to break even when compared to traditional technologies. Scenarios in which the natural gas price is low allow break-even cost for both individual hydrogen units and the assembled modular plant. The economy of learning must produce a reduction of $40 \%$ or less in capital cost when the natural gas price is under $0.02 \mathrm{US} \$ / \mathrm{Sm}^{3}$. This result suggests that the synthesized modular hydrogen process has potential to be economically feasible under these conditions. The developed tools can be used to accelerate the deployment and manufacturing of standardized modular energy systems. 


\section{Acknowledgments}

I first thank Dr. Fernando V. Lima for the advice and encouragement. The early introduction to WVU opened the way to this Ph.D. work by awakening my interests in science and research. This accomplishment has only been possible through his consistent support.

The undergraduate research that I performed at Universidade de São Paulo gave me a solid background in research methodology. For that, I thank Dr. Marcelo Seckler and Dr. José Luis Pires de Camacho. My deepest gratitude to Dr. David R. Vinson for his patience, collaboration, and support through all the steps of my Ph.D. I truly appreciate the optimization instructions, insights about computational methods, fundamental literature recommendations and discussions on industry and manufacturing provided by Dr. Debangsu Bhattacharyya, Dr. YanFang Ye, Dr. Stephen E. Zitney and Dr. Thorsten Wuest, respectively.

I would like to acknowledge my colleagues from the Control, Optimization and Design for Energy and Sustainability (CODES) research group, who were open to discussions and help, especially, Dr. Juan Carlos Carrasco. I also thank Hannah, Zach, Carlie, Maddie, San, Thiago, Brent, Selorme, Ron, Franklin David, Dr. Mirlekar, and Dr. He Xin. I appreciate the contribution from Dr. Heleno Bispo, Victor Alves and Ícaro Chagas.

I am grateful for the unconditional support to pursue an advanced degree offered by my parents Alexandre and Regina, and my brother Bruno. My closest friend Rebecca H. H. Kim has walked alongside me through this journey since even before our B.S. Her friendship, empathy, honesty, and loyalty gave me strength to keep pushing through. I thank my friends George Teixeira, Caio Mecca, and Jordan Chapman for keeping in touch regardless of adversities. To my partner Jonathan Thompson, I thank for all the love and support in these last two years.

Financial support from American Chemical Society - Petroleum Research Fund (ACS-PRF), National Science Foundation (NSF) and U.S.-China Clean Energy Research Center (CERC) program allowed the completion of this project. 


\section{Table of Contents}

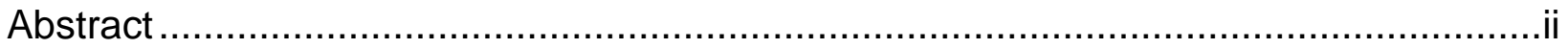

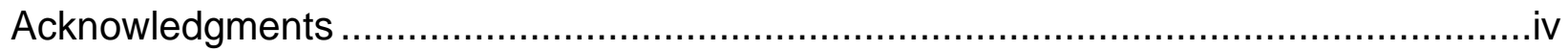

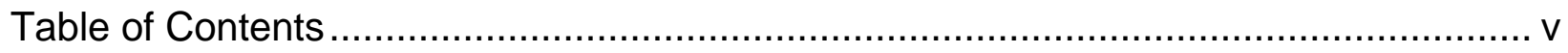

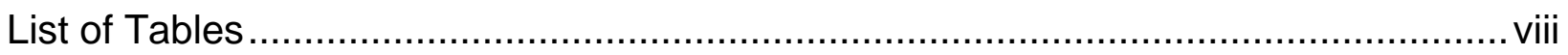

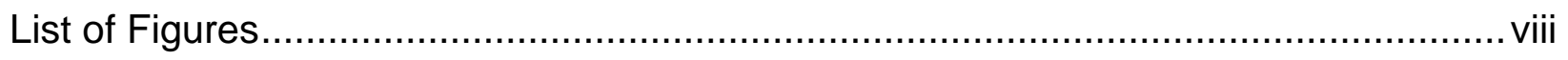

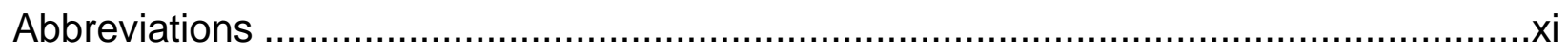

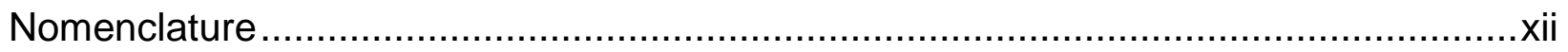

Chapter 1 Introduction and Problem Statement .................................................... 2

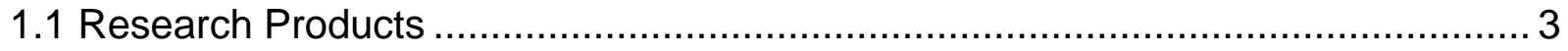

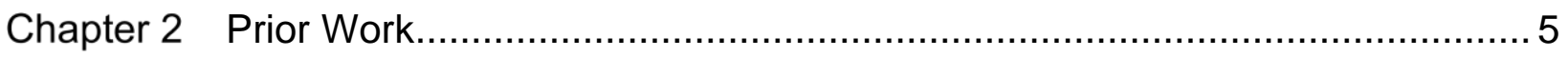

2.1 Evolution of Process Operability Field ……….............................................. 5

2.2 Process Operability Concepts...................................................................... 7

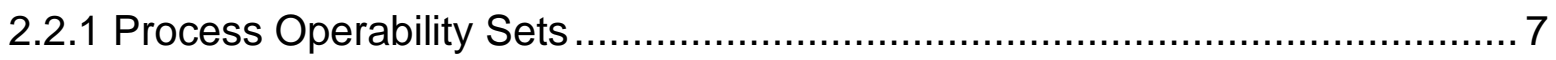

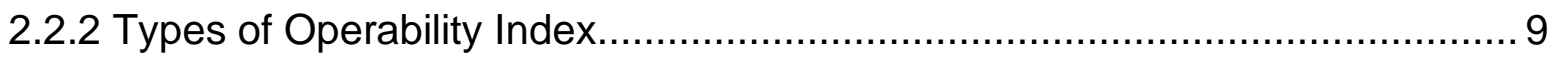

2.3 Modular and Intensified Energy Systems Operability ..................................... 11

2.4 Modular Systems Costing Challenges ......................................................... 13

2.5 Previous Nonlinear Programming-based Operability Approaches ....................... 15

2.6 Illustration of Process Operability Concepts: Classic Shower Problem ............... 17

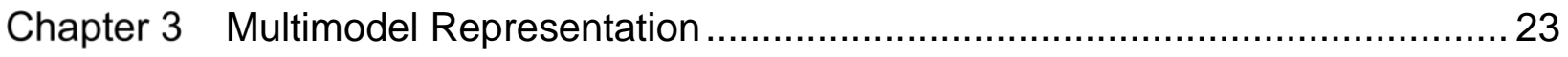

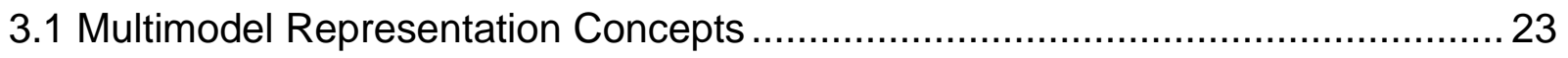

3.1.1 Input-Output Representation without Disturbances .................................. 23

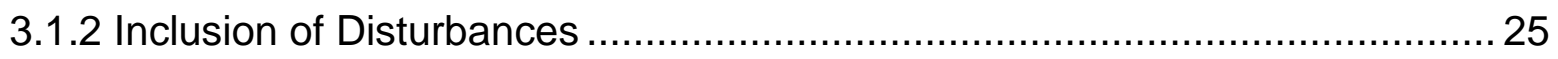

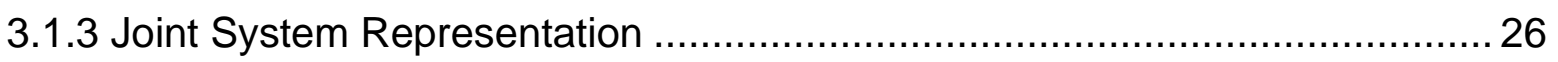

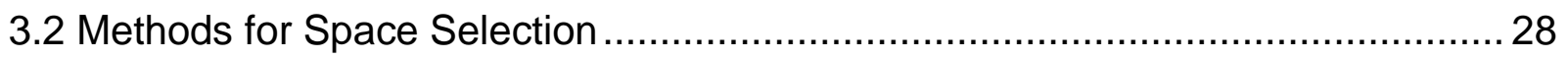




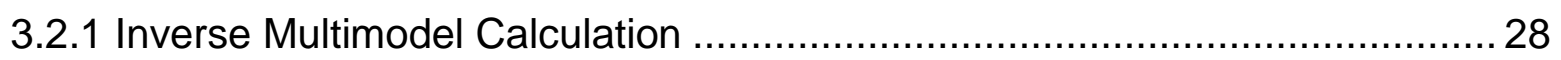

3.2.2 Iterative Mixed Integer Linear Programming-based Algorithm ...................... 30

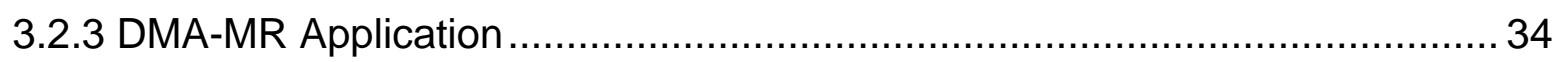

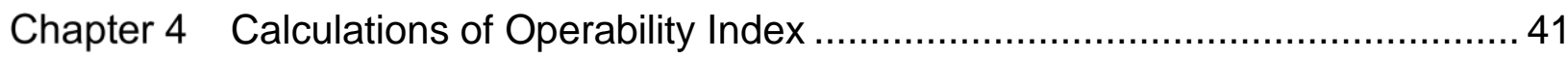

4.1 Calculations with Hypervolumes and Subregions ......................................... 41

4.2 Inclusion of Disturbances in Operability Index Calculations............................... 44

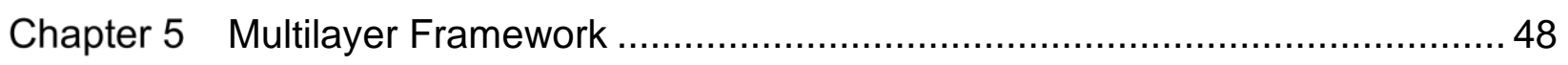

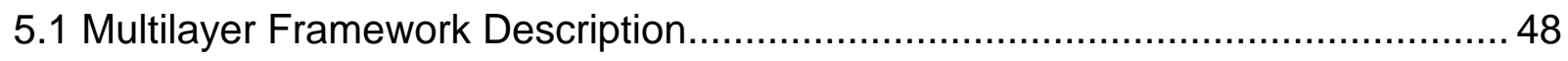

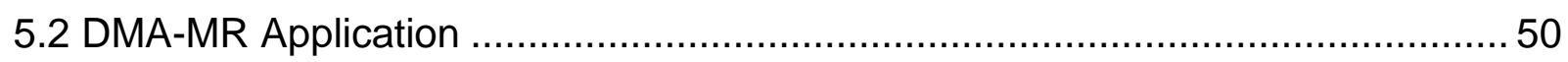

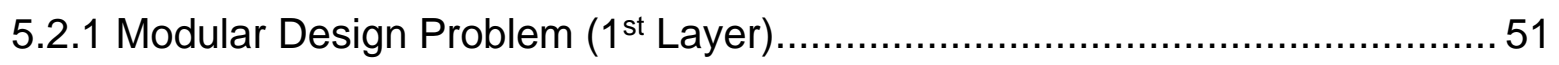

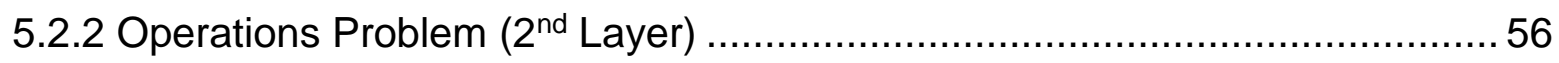

5.2.3 MDR Ranking using Subregions and Hypervolumes ................................ 59

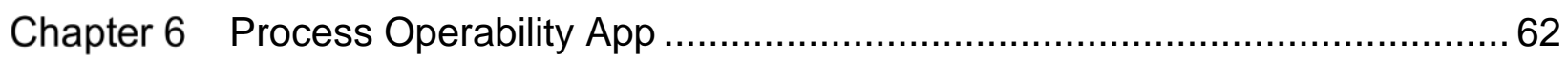

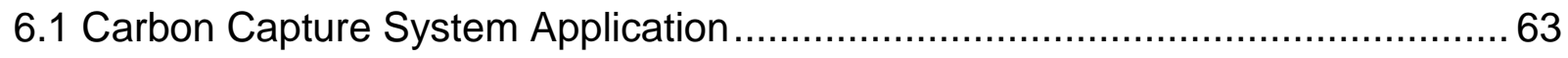

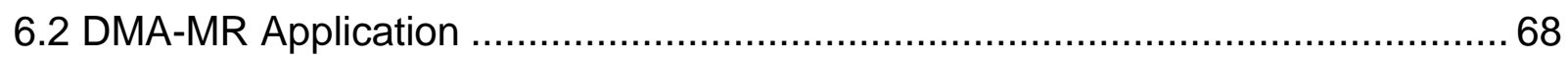

Chapter 7 Modular Cost and Operability Analysis for Technology Deployment ..........71

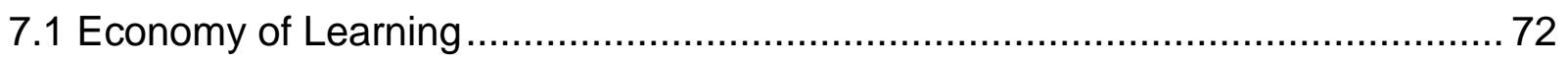

7.2 Modular Techno-Economic Analysis Framework ............................................. 73

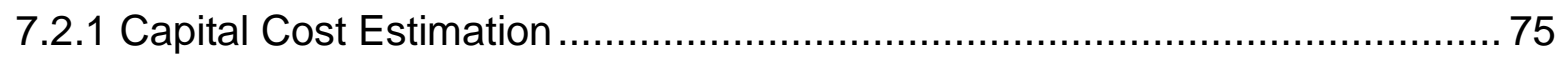

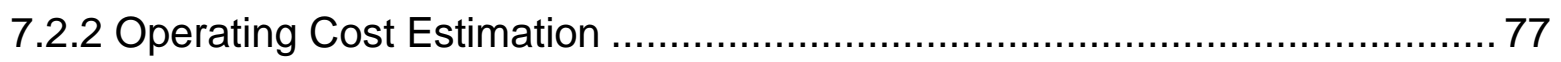

7.2.3 Base Case Scenario Cost Estimation .................................................... 79

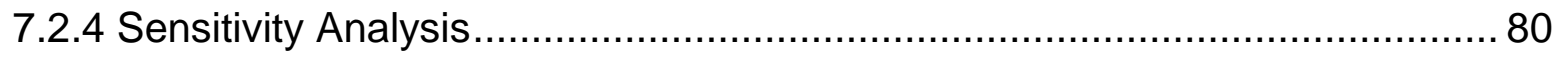

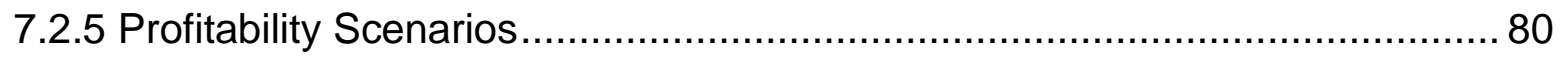

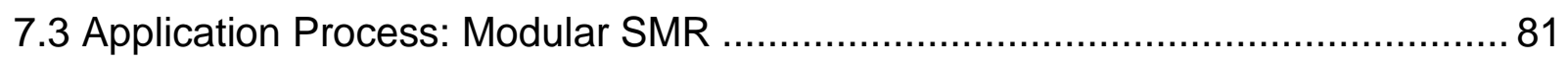

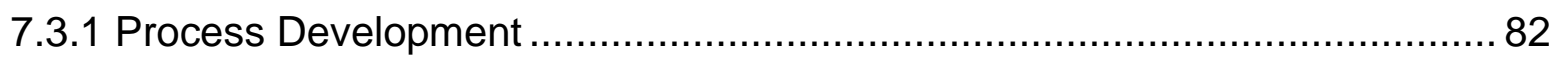


7.3.2 Selection of Nominal Design and Operation............................................ 84

7.3.3 Application of Modular Techno-Economic Analysis Framework .................... 88

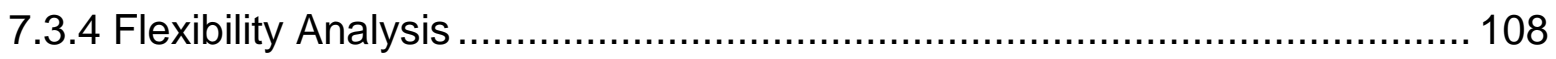

Chapter 8 Conclusion and Recommendations .................................................... 114

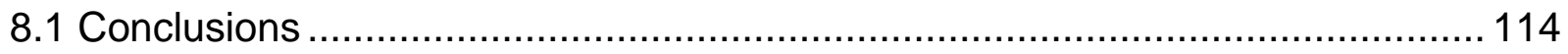

8.2 Recommendations and Future Work …….................................................. 116

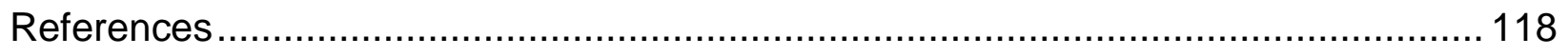

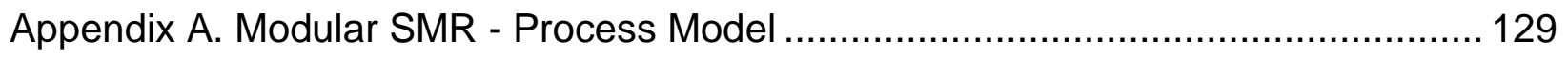

Appendix B. Modular SMR - Equipment Cost Calculation ....................................... 131 


\section{List of Tables}

Table 1. Dimensional limits for transportation of modular systems by road................... 12

Table 2. Design input variables and available ranges. ............................................... 36

Table 3. Operational input variables and available ranges........................................... 36

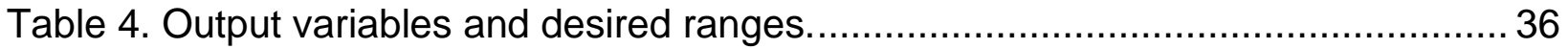

Table 5. Input variables and available ranges for CCS application. ............................. 64

Table 6. Operational input variables and available ranges......................................... 86

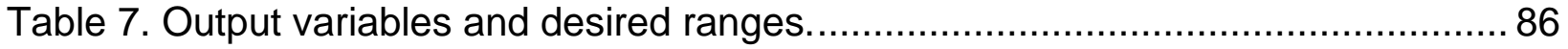

Table 8. Estimated modular bare module cost. ........................................................ 90

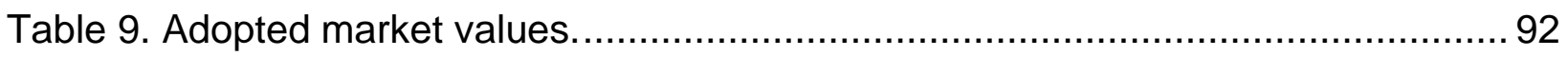

Table 10. Design input variables and available ranges. ......................................... 109

Table A.1. Description of main process streams. ................................................. 130

Table B.1. Estimation of purchase and bare module costs. .................................... 133

\section{List of Figures}

Figure 1. Calculations of $\mathrm{s}-\mathrm{Ol}$ for the shower problem. ........................................... 19

Figure 2. System behavior with respect to regulatory action.................................... 20

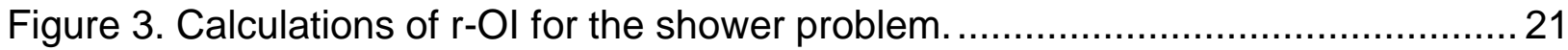

Figure 4. System behavior with respect to a collection of regulatory actions. ...............2 21

Figure 5. Calculations of o-Ol for the shower problem. ........................................... 22

Figure 6. Illustration of the multi-model polytope representation for a $2 \times 2$ system...... 25

Figure 7. 2x1 example: (a) disjoint system representation and (b) joint system

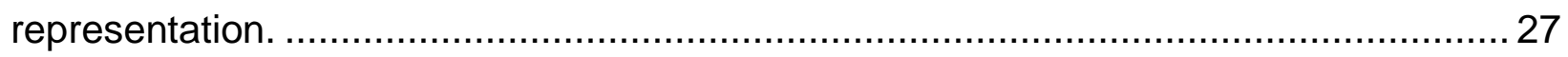

Figure 8. Inverse multimodel application to the shower problem (servo case): (a) without triangulations and (b) with triangulations and interpolations...................................... 30

Figure 9. Steps of the MILP-based iterative approach. ............................................. 31

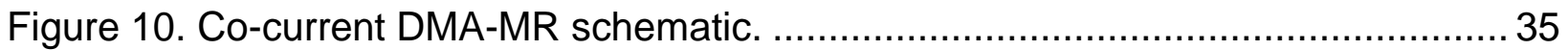

Figure 11. Two-dimensional case: (a) input variable bounds and triangulation; (b) selection of triangles and optimal solution points for each iteration............................. 40 
Figure 12. Two-dimensional case: (a) output mapping; (b) selection of triangles and optimal solution points for each iteration.

Figure 13. s-OI with multimodel representation for shower problem: (a) AIS; (b) AOS and $\mathrm{s}$-OI subregions; (c) AOS and s-OI hypervolumes. 42

Figure 14. Calculation of output sets in terms of: (a) subregions; and (b) hypervolumes. .

Figure 15. Multimodel calculations of $\mathrm{r}$-OI for the shower problem. .......................... 45

Figure 16. Multimodel calculations of 0 -Ol for the shower problem............................. 46

Figure 17. Cuts of the AISop'-AOS' mapping for the shower problem........................... 47

Figure 18. Multilayer framework scheme.......................................................... 48

Figure 19. Three-dimensional: Input variable bounds and triangulations. .................... 54

Figure 20. Three-dimensional: Selection of tetrahedra and optimal solution points. ..... 54

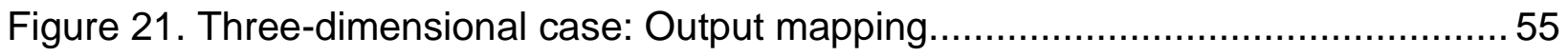

Figure 22. Three-dimensional: Selection of tetrahedra and optimal solution points. ..... 55

Figure 23. s-Ol and model inversion for design of highest s-Ol. ............................... 57

Figure 24. s-Ol and model inversion for previously obtained optimal design. ............... 58

Figure 25. Achievability analysis of MDR designs using s-Ol..................................... 58

Figure 26. Classification of MDR using s-Ol in terms of subregions. ........................... 60

Figure 27. Classification of MDR using s-OI in terms of hypervolumes.........................6 60

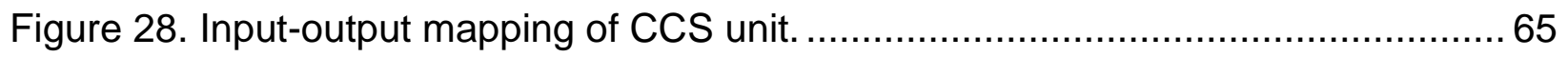

Figure 29. Steady-state achievability of $90 \%$ of $\mathrm{CO}_{2}$ capture rate................................ 66

Figure 30. Steady-state achievability of $92 \%$ of $\mathrm{CO}_{2}$ capture rate............................... 67

Figure 31. DOS* (color coded points) and intensified point for DMA-MR 7x3 application.

Figure 32. Illustration of the experience curve considering $\alpha=0.15$ and $\operatorname{Rmax}=30 \% .73$

Figure 33. Summarized step-by-step modular TEA framework.................................. 75

Figure 34. Experimental scheme of consecutive microchannels.................................. 82

Figure 35. Simplified block flow diagram: modular hydrogen unit. .............................. 83

Figure 36. Process flow diagram: modular hydrogen unit (adapted from Aspen Plus). . 84

Figure 37. AIS op and feasible operating input region............................................. 87

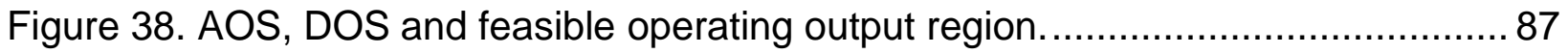


Figure 39. Simplified block flow diagram: conventional SMR plant. 88

Figure 40. Experience curve: model fitting. Literature literature data points from ref. ${ }^{56.93}$ Figure 41. Comparison of scaled costs: EAOC, total capital cost and operating cost. .. 94

Figure 42. Detailed comparison of scaled operating costs. 95

Figure 43. Sensitivity analysis of scaled EAOC of modular unit: (a) Rn, amod and $P N G$, mod and (b) lfmod and irmod. 98

Figure 44 . Achievability of profitability scenarios. Faded gray lines represent values from the base case. 101

Figure 45. Differences between scaled EAOC of a unit and of the plant (cumulative) $P N G=0 \mathrm{US} \$ / \mathrm{Sm} 3$. Dotted faded lines correspond to the number of units 92 , required to achieve regular hydrogen production. 104

Figure 46. Mapping of leaning parameters for NG price scenarios. Base case lines represent values of $\alpha=0.24$ and $\operatorname{Rmax}=42 \%$, modeled after literature. 106 Figure 47. Differences between scaled EAOC of a unit and of the plant (cumulative) $P N G=0.1119$ US $\$ / S m 3$. Dotted faded lines correspond to the number of units of 92 , required to achieve regular hydrogen production. 107

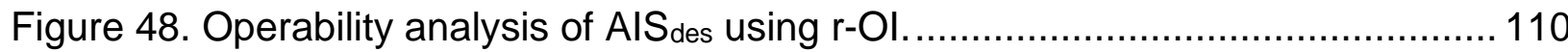

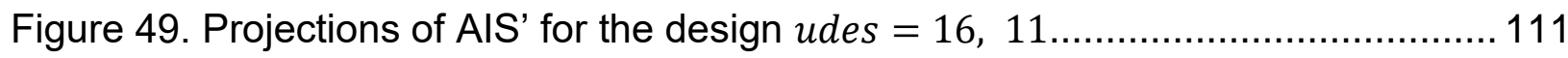

Figure 50. Projections of AOS' for the design udes $=16,11 \ldots \ldots \ldots \ldots \ldots \ldots \ldots \ldots \ldots \ldots \ldots . . .111$

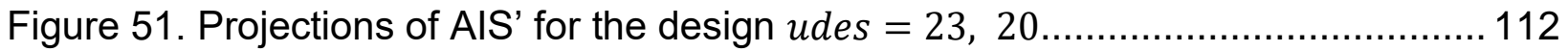

Figure 52. Projections of AOS' for the design udes $=23,20 \ldots \ldots \ldots \ldots \ldots \ldots \ldots \ldots \ldots \ldots \ldots . . .113$ 


\section{Abbreviations}

\begin{tabular}{ll} 
AIS & Available Input Set \\
AOS & Achievable Output Set \\
BL & Boundary Limit \\
CCS & Carbon Capture System \\
COM & Cost of Manufacture (Operating Cost) \\
CV & Controlled Variable \\
DIS & Desired Input Set \\
DMA-MR & Direct Methane Aromatization - Membrane Reactor \\
DOS & Desired Output Set \\
EAOC & Equivalent Annual Operating Cost \\
EDS & Expected Disturbance Set \\
MDR & Modular Design Region \\
MEA & Monoethanolamine \\
MILP & Mixed-Integer Linear Programming \\
MV & Manipulated Variable \\
NG & Natural Gas \\
NLP & Nonlinear Programming \\
ODE & Ordinary Differential Equation \\
OI & Operability Index \\
o-OI & Overall Operability Index \\
PI & Process Intensification \\
PSA & Pressure Swing Adsorption \\
r-OI & Regulatory Operability Index \\
SM & System Modularization \\
SMR & Steam Methane Reforming \\
s-OI & Servorability Index \\
\hline
\end{tabular}


TEA Techno-Economic Analysis

\section{Nomenclature}

a

C

d

D

$\mathrm{F}$

$\mathrm{Fr}$

ir

$\mathrm{L}$

If

m

M

$M^{-1}$

$\mathrm{n}$

OP

$\mathrm{P}$

$\mathrm{p}$

PA

q

$\mathrm{R}$

S

$S^{\prime}$

S"

$\mathrm{Sm}^{3}$

$\mathrm{T}$ contingency and fees

purchase cost

disturbances

inner tube diameter

purchase cost factor

frontier of a set operator

Interest rate

reactor length

plant lifetime

number of inputs

process model

inverse model

number of manufactured modular units

annual plant operating time

polytope

number of outputs

present to annuity factor

number of disturbances

reduction in purchase cost

set that counts polytopes

set with counts of polytopes that achieve $\operatorname{Fr}(D O S)$

set with counts of polytopes that do not achieve $\operatorname{Fr}(\mathrm{DOS})$

Standard $\mathrm{m}^{3}$

production of a chemical of interest 
u

y

$\alpha$

\section{Subscripts}

BM

CAT

con

des

EAOC'

mod

MP

n

$\mathrm{OL}$

op

sol

TM

URM

WT inputs

outputs

experience rate exponent

bare module

catalyst

conventional technology

design variables

scaled EAOC

modular technology

assembled modular plant

$\mathrm{n}^{\text {th }}$ manufacture modular unit

operating labor

operational (manipulated) variables

polytope of solution

total module

utilities and raw material

waste treatment 


\section{Superscripts}

$\begin{array}{ll}* & \text { feasible } \\ & \text { All with EDS appended (e.g. AIS'=AIS x EDS) } \\ & \text { time derivative } \\ \text { MP } & \text { modular plant } \\ \mathrm{N} & \text { nominal point } \\ \mathrm{u} & \text { input polytope } \\ \mathrm{u}, \mathrm{d} & \text { polytope in AIS' } \\ \mathrm{y} & \text { output polytope } \\ \mathrm{y}, \mathrm{d} & \text { polytope in AOS' }\end{array}$




\section{MULTIMODEL OPERABILITY APPROACH FOR DESIGN OF MODULAR AND INTENSIFIED ENERGY SYSTEMS}




\section{Chapter 1 Introduction and Problem Statement}

Novel small, modular, and intensified energy systems are emerging technologies that show promising capabilities for the conversion of raw materials and resources into valueadded chemicals and energy. Transportable modular units can monetize resources available in conditions that are prohibitive to the construction of traditional large-scale plants. Drastic reductions in physical size are considered for the conceptualization of these technologies, making them susceptible to demanding operations and process control efforts during their implementation phase. Also, modular process design may lack well-established guidelines and heuristics for feasible operation. Coupling effects and loss of degrees of freedom have been observed, especially when process integration and intensification strategies are employed for designing modular energy systems. ${ }^{1}$ Additional challenges may arise from system nonlinearities and highly constrained environments that are present in the process models required to represent these systems.

As a solution, process operability methods can be used during the design phase to foresee and address such operational challenges, ensuring feasibility, optimality, and controllability. ${ }^{2}$ Nevertheless, operability approaches are historically tailored to specific systems or applications. The availability of readily accessible and versatile operability algorithms is still limited, consisting of a gap in the known literature. Another challenge yet to be addressed is the absence of techno-economic and profitability aspects incorporated into process operability analyses.

This research aims to develop an operability framework that systematically ranks modular design candidates according to operational performance. A concise measure of operability index $(\mathrm{Ol})$ is employed to classify available designs. The OI measure alleviates future control efforts, by indicating design conditions that provide improved flexibility and controllability. Process nonlinearities are represented by multiple linearized models that are produced using computational geometry techniques. An optimization-based multilayer operability framework addresses the problems of minimization of process footprint, while attaining process intensification $(\mathrm{PI})$ targets. An open-source process operability app is developed for easy and quick dissemination of the available operability 
methods. A modular techno-economic analysis (TEA) that is based on the economy of learning is also proposed to analyze the economic viability of modular systems. A variety of applications demonstrate effectiveness of the developed framework, including the classical shower problem in process operability, a membrane reactor for direct methane aromatization conversion to hydrogen and benzene (DMA-MR), and a newly synthesized modular hydrogen unit.

The structure presented in this dissertation is as follows. Initially, related previous work is described. Then, the system representation with multiple models is explained, as well as methods for space selection. Calculations of OI are presented, followed by the multilayer operability framework. The process operability app is introduced, and the modular TEA framework is proposed. Finally, conclusions are drawn, and recommendations are presented.

\subsection{Research Products}

The presented work contributions have resulted in the following peer-reviewed publications:

(1) Gazzaneo, V., Watson M., Ramsayer C. B., Kilwein Z. A., Lima, F. V. A TechnoEconomic Analysis Framework for Intensified Modular Systems. In preparation.

(2) Gazzaneo, V.; Carrasco, J. C.; Vinson, D. R.; Lima, F. V. Process Operability Algorithms: Past, Present, and Future Developments. Ind. Eng. Chem. Res. 2020. ${ }^{3}$

(3) Gazzaneo, V.; Lima, F. V. Multilayer Operability Framework for Process Design, Intensification, and Modularization of Nonlinear Energy Systems. Ind. Eng. Chem. Res. 2019. ${ }^{4}$

(4) Gazzaneo, V.; Carrasco, J. C.; Lima, F. V. An MILP-Based Operability Approach for Process Intensification and Design of Modular Energy Systems. In Computer Aided Chemical Engineering; 2018. ${ }^{5}$

The following presentations were also outcomes of the developed work:

(1) Watson M., Gazzaneo V. and Lima F. V. "Operability Analysis for Design and Control of a Modular Steam Methane Reforming Process", Virtual AIChE Annual Meeting, November (2020). 
(2) Gazzaneo V. and Lima F. V. "A Process Operability App for Intensification and Modularization of Energy Systems", Virtual AIChE Annual Meeting, November (2020).

(3) Gazzaneo V. and Lima F. V. "A Process Operability App for Intensification and Modularization of Energy Systems" Bio Processes, Control, Optimization, and Industry 4.0 Online Conference (2020).

(4) Ramsayer B. C., Gazzaneo V. and Lima F. V. "Economic Analysis of Modular SteamMethane Reforming Reactors Using Economy of Numbers", West Virginia Undergraduate Research Day at the Capitol, Charleston, WV, February (2020).

(5) Gazzaneo V. and Lima F. V. "Development of Process Operability Algorithms for Modularization and Intensification of Energy Systems", AIChE Annual Meeting, Orlando, FL, November (2019).

(6) Kilwein Z., Gazzaneo V. and Lima F. V. "Modeling and Techno-Economic Analysis of a Modular Hydrogen Production Process", AIChE Annual Student Conference (1st place award), Pittsburgh, PA, November (2018).

(7) Gazzaneo V. and Lima F. V. "An MILP-Based Operability Approach for Process Design, Intensification and Modularity of Nonlinear and High-Dimensional Energy Systems", AIChE Annual Meeting, Pittsburgh, PA, November (2018).

(8) Gazzaneo V., Carrasco J. C. and Lima F. V. "An MILP-based Operability Approach for Process Intensification and Design of Modular Energy Systems", 13th International Symposium on Process Systems Engineering (PSE 2018), San Diego, CA, November (2018).

(9) Gazzaneo V., Carrasco J. C. and Lima F. V. "Multi-model Operability Approach for Process Design, Intensification and Modularity: Application to Nonlinear and HighDimensional Membrane Reactors", AIChE Annual Meeting, Minneapolis, MN, November (2017).

Presentations (7) and (8) were associated with the AIChE Best Presentation in Session "Process Intensification through Process Systems Engineering" and PSE 2018 Young Researcher Travel Award, respectively. Also, Zachary Kilwein was awarded CAST Overall Winner at the 2018 AIChE Undergraduate Student Poster Competition for presentation (6).

The software infrastructure contribution in this dissertation is an open-source platform named "Process Operability App Project" in MATLAB, available at:

\section{https://fernandolima.faculty.wvu.edu/operability-app}




\section{Chapter 2 Prior Work}

Process operability was originally proposed to incorporate control aspects into process design of chemical systems. ${ }^{2}$ One example of a nonlinear chemical system corresponds to the development of new modular and intensified energy systems. A summary of prior work on process operability analysis and its application to development of modular and intensified energy systems is provided in this chapter.

\subsection{Evolution of Process Operability Field}

Process operability analysis emerged as a viable alternative to the sequential tasks of process design, followed by the selection of control strategies. Such tasks were integrated in early design stages to assure optimality and feasibility. The concept of Ol was introduced as a measure of the capability of a design to handle operational uncertainties, disturbances, and process constraints. This concept was widely applied to steady-state systems to give insights in the process synthesis phase about future plant operations. ${ }^{2,6,7}$ Dynamic operability approaches were also developed to extend steady-state operability concepts and assess transient output constraints. ${ }^{7-9}$ These approaches produced a performance upper bound for achieving operating regions, derived in terms of an idealized controller scenario. In this dissertation, focus will be given to steady-state operability analysis. A review of dynamic operability can be found in ref. ${ }^{10}$.

Throughout the development of operability methods, input-output mappings have always been present as an indispensable technique to quantify achievability and controllability. When performing this mapping, intrinsic challenges such as space nonconvexities, nonlinearities, and system dimensionality were brought into the operability analysis. Historically, several approaches were developed to tackle these challenges, mainly either in the field of operability or in the field of flexibility such as (i) response surface-based techniques (Kriging and response surface methods), ${ }^{11,12}$ (ii) data-driven experiments and design of experiments, ${ }^{11-14}$ (iii) optimization-based algorithms, ${ }^{15-18}$ (iv) high-dimensional data-driven model representations, ${ }^{12,19}$ (v) parametric and multiparametric approaches, ${ }^{20,21}$ (vi) metamodeling, ${ }^{22}$ (vii) simplicial 
approximation, ${ }^{19}$ (viii) cylindrical algebraic decomposition, ${ }^{23-25}$ and (ix) approximation by convex shapes. ${ }^{26-28}$

Feasibility, flexibility, controllability, resilience, and reliability have been widely associated with process operability. ${ }^{9,29-32}$ All terms refer to the evaluation and improvement of process operations with respect to unexpected changes from a given nominal point. Feasibility is associated with the viability of a process, and a common goal is to find a feasible region of operation. The other terms can be classified by how process disturbances are defined. Flexibility and controllability usually measure the process ability to reject disturbances and move from one setpoint to another, respectively. Resilience and reliability are often associated with risk of failure and analysis of the system behavior with respect to design uncertainty, stability, and exogenous disturbances. These terms may be complementary or interchangeable depending on how problems are defined and addressed. ${ }^{33}$ Other definitions may arise, especially when particularities of dynamic performance are considered. ${ }^{34-36}$

Process operability can be viewed as an umbrella term, and the previous definitions as particular cases. Process operability is independent of the defined control structure and provides a systematic methodology to analyze intrinsic characteristics of nonlinear systems. ${ }^{6}$ Traditionally, disturbances, available degrees of freedom, constraints, and desired production goals are translated into mathematical sets for steady-state process operability analysis. Set-theory operations provide measures of OI that correspond to the systems' capabilities mentioned above. Subsection 2.2 describes how process feasibility is evaluated in terms of achievability of a desired operation with and without the presence of disturbances. Types of $\mathrm{Ol}$ calculation are explained considering manipulated and controlled variables and ability of the system to fulfill servo and regulatory control objectives. 


\subsection{Process Operability Concepts}

Set-point operability was originally introduced for controllability analysis of square systems. ${ }^{2}$ Considering a system with $m$ inputs, $p$ outputs and $q$ disturbances, the following process model, denoted by $M$, can describe the system behavior:

$$
M=\left\{\begin{array}{l}
\dot{x}=f(x, u, d) \\
y=g(x, u, d) \\
h_{1}(\dot{x}, x, y, \dot{u}, u, d)=0 \\
h_{2}(\dot{x}, x, y, \dot{u}, u, d) \geq 0
\end{array}\right.
$$

where $x \in \mathbb{R}^{n}$ are the state variables, $y \in \mathbb{R}^{p}$ are the outputs, $u \in \mathbb{R}^{m}$ are the inputs, $d \in$ $\mathbb{R}^{q}$ are the disturbances. $f: \mathbb{R}^{m+n+q} \rightarrow \mathbb{R}^{n}$ and $g: \mathbb{R}^{m+n+q} \rightarrow \mathbb{R}^{n}$ are nonlinear maps. $h_{1}$ and $h_{2}$ are equality and inequality process constraints, respectively. Furthermore, $\dot{x}$ and $\dot{u}$ represent time derivatives associated with $x$ and $u$, respectively. This notation is used throughout this dissertation to describe process operability sets and distinct types of OI.

\subsubsection{Process Operability Sets}

With the above notation, three basic operability sets can be readily defined, as follows.

Available Input Set (AIS). This set of available inputs may be changed within a certain range according to accessibility. It can represent operational inputs and/or design inputs. Operational inputs are manipulated variables (MVs), the subject of control studies, whereas design inputs are associated with available designs (available material, dimensions, etc.). Mathematically, the AIS is given by

$$
A I S=\left\{u \in \mathbb{R}^{m} \mid u^{\min } \leq u \leq u^{\max }\right\}
$$

When needed, the AIS can be distinguished by sets that solely comprise design variables $\left(A I S_{\text {des }} \in \mathbb{R}^{m_{\text {des }}}\right)$, or operational variables $\left(A I S_{o p} \in \mathbb{R}^{m_{o p}}\right)$, in which $m_{\text {des }}$ and $m_{o p}$ are the dimensionalities of $A I S_{d e s}$ and $A I S_{o p}$, respectively. In case both types of input variables are present, the complete AIS is a result of the Cartesian product $A I S_{d e s} \times A I S_{o p}$ with $m=$ $m_{\text {des }}+m_{o p}$. 
Desired Output Set (DOS). This set represents the desired region of operation. It may be defined, for example, by process constraints and desired production or efficiency. Mathematically, the DOS is given by

$$
\text { DOS }=\left\{y \in \mathbb{R}^{p} \mid y^{\min } \leq y \leq y^{\max }\right\}
$$

Insights about the determination of the DOS may be given by input-output mappings using the AIS and the Achievable Output Set (AOS) defined below. For example, given an AIS that represents available process conditions for MVs, an AOS with achievable controlled variables (CVs) can be generated through direct mapping. By inspecting the generated AOS, achievable zones may be analyzed for selecting the best operating output region according to economic or environmental targets. Experimental recommendations are also important factors in the selection of the DOS: system pressure and temperature limits should not be exceeded in order to preserve mechanical and chemical integrity of structures (e.g., structure of metals for catalysts, membranes, and so on). If desired, the DOS can be defined by a single desired point as

$$
\text { DOS }=y^{N}
$$

where $y^{N} \in \mathbb{R}^{p}$ represents a setpoint, or a desired nominal output operation point.

Expected Disturbance Set (EDS). This is a set of expected disturbances, also representing process uncertainties, which is mathematically defined as

$$
E D S=\left\{d \in \mathbb{R}^{q} \mid d^{\text {max }} \leq d \leq d^{\text {max }}\right\}
$$

For a system without perturbations (i.e., disturbances are kept in their nominal values), the EDS is defined by

$$
E D S=d^{N}
$$

where $d^{N} \in \mathbb{R}^{q}$ is a vector composed by fixed nominal values.

Considering the process model $M$, the inverse model $M^{-1}$, and the sets above, other operability sets can be calculated as described below. 
Achievable Output Set (AOS). This is a set of CVs that the system can achieve for the considered AIS and EDS. This set is generically defined as a function of inputs and disturbances by

$$
A O S=\left\{y \in \mathbb{R}^{p} \mid y=M(u, d), u \in A I S \text { and } d \in E D S\right\}
$$

Different cases can be analyzed depending of the operability goals and definitions of EDS and DOS.

Desired Input Set (DIS). This is a set of required inputs needed to achieve the entire DOS. It can be computed by applying the inverse model to all the elements in the DOS. This set is generically described by

$$
D I S=\left\{u \in \mathbb{R}^{m} \mid M(u, d)=y, d \in E D S \text { and } y \in D O S\right\}
$$

Similarly, cases may arise depending on definitions of EDS and DOS. When needed, the DIS can also be distinguished as $D I S_{d e s}$ and DIS $S_{o p}$. Subsection 2.2.2 describes how different scenarios and objectives influence the formulations of AOS and DIS along with distinct types of OI.

\subsubsection{Types of Operability Index}

The operability sets above are used to calculate measures of OI. Different types of OI can be calculated using the subsets associated with manipulated and CVs. Each type of OI and respective goal is explained, as follows.

Servo-OI (s-OI). Without regulatory control and with disturbances at their nominal values, the s-OI measures the capability of a system to perform servo control operations. The DOS and EDS are defined by Equations (3) and (6), corresponding to a region of setpoints and a nominal value for the disturbance, respectively. The s-Ol can be estimated from input or output perspectives respectively by

$$
\begin{gathered}
s-O I=\frac{\mu\left(A I S_{o p} \cap D I S_{o p}\right)}{\mu\left(D I S_{o p}\right)} \\
s-O I=\frac{\mu(A O S \cap D O S)}{\mu(D O S)}
\end{gathered}
$$


where the letter $\mu$ corresponds to a measure of the depicted region. For one dimension, $\mu$ is simply a measure of length; for two dimensions, area; for three dimensions, volume and for higher-dimensional cases, hypervolume. When calculated from the input perspective using Equation (9), the s-OI indicates what percentage of required inputs achieve a desired operation that is fulfilled by the AIS. Whereas an s-OI that is calculated from an output perspective using Equation (10) indicates the percentage of the desired operation that is achieved.

Regulatory-OI ( $\mathbf{r}-\mathrm{Ol})$. When disturbances are present, and there is only one setpoint, the $\mathrm{r}-\mathrm{O}$ l measures the ability of the system to stay at the desired setpoint by performing changes in the MVs. The DOS and EDS are defined by Equations (4) and (5) corresponding to expected disturbance region and to a desired setpoint, respectively. The $\mathrm{r}$-OI can be estimated from the input perspective using Equation (9). The obtained DISop contains the required combinations of $\mathrm{MVs}$ to compensate disturbances. If desired, the $\mathrm{r}$ OI can be calculated through output perspective using Equation (10). However, the AOS must be changed to depict a region that is always achievable, regardless of the presence of disturbances, given by

$$
A O S=\bigcap_{d \in E D O S} A O S(d)=\left\{y \in \mathbb{R}^{p} \mid y=M(u, d), u \in A I S_{o p} \text { for all } d \in E D S\right\}
$$

Overall-OI (o-OI). When disturbances are present and a region of setpoints is desired, the o-Ol measures the ability of the system to perform changes in the MVs and stay at each desired setpoint of that region. The EDS and DOS are defined by Equations (3) and (5), corresponding to regions of desired setpoints and expected disturbances, respectively. Similarly, the $0-\mathrm{Ol}$ is estimated from the input perspective using Equation (9). A direct consequence of the employed EDS and DOS is an enlarged DIS op region in comparison to the one from $\mathrm{r}$-OI. In that case, the DISop comprises all required combinations of MVs to compensate for all disturbances and keep the system at each desired setpoint. If desired, the o-OI can be calculated from the output perspective using Equation (10). In that case, the AOS must also be changed using Equation (11).

Operability methodologies usually require calculation of DIS for estimation of r-OI and o-OI. Elements of DISop can be obtained based on combinations of $y \in D O S$ and $d \in$ 
$D I S_{o p}$ and an inverse model described by $M^{-1}: \mathbb{R}^{p+q} \rightarrow \mathbb{R}^{m}$. However, the derivation of $M^{-1}$ may be complex and sometimes not straightforward because nonlinear systems require a nonlinear model $M$. In this work, the numerical computation of $M^{-1}$ is performed using computational geometry tools, overcoming the challenges associated with the analytical calculation of $M^{-1}$. Moreover, alternative calculations of $\mathrm{r}-\mathrm{Ol}$ and $\mathrm{o}-\mathrm{Ol}$ are proposed based only on the direct mapping $M$ shown in Chapter 4 .

For nonsquare systems with more outputs than inputs, the above concepts can be adapted for interval operability. The study of output constraints is of particular importance for approaches that address this variation of process operability. More information about interval operability can be found in ref. ${ }^{37}$.

\subsection{Modular and Intensified Energy Systems Operability}

System modularization (SM) and $\mathrm{PI}$ are commonly achieved by integrating unit operations and adopting new enhanced reaction and separation strategies. As a result, highly integrated and customized designs lack guidelines on how to assure a feasible and profitable operation. $\mathrm{PI}$ is a comprehensive set of strategies to reduce the size of chemical processes, producing efficient designs in terms of conversion, consumption of utilities and energy, emissions, and waste disposal. The concepts of modularization in turn are usually limited to spatial configuration and the ability to manufacture a plant in the form of standardized modules for skid-mounted assembly and easy transportation. In this case, size and weight limitations should be compatible to maritime, rail or road transportation.

The way in which the modules are transported mainly depends on the place and location where modules are built, typically close to where they will be installed. For example, in the scope of recovering natural gas (NG) from the Marcellus Shale Formation in the states of WV and PA, shipment can be facilitated by road access, resulting in dimension limitations consistent with commercial trucks, ${ }^{38,39}$ shown in Table 1.40 
Table 1. Dimensional limits for transportation of modular systems by road.

\begin{tabular}{ll}
\hline dimension & limit \\
\hline Width & $8.5 \mathrm{ft} .(2.5 \mathrm{~m})$ \\
Height ${ }^{a}$ & $8.5 \mathrm{ft} .(2.5 \mathrm{~m})$ \\
Length & $53 \mathrm{ft} .(16.1 \mathrm{~m})$ \\
Volume & $3,829 \mathrm{ft}^{3}\left(108 \mathrm{~m}^{3}\right)$ \\
Weight &
\end{tabular}

aFrom the flatbed trailer to the top of the load (assuming $5 \mathrm{ft}$. tall flatbed). ${ }^{b}$ Excluding the average vehicle weight of approx. 32,000 lb (i.e., 80,000 lb total)

Design flexibility is key to ensure feasible onsite operation as modular units are moved from one location to another. The development of flexible and intensified modular designs has been a focus of recent studies. ${ }^{41}$ Conventional process operability has also been modified to analyze new flexible designs that employ the concepts of PI and SM. Design variables were included as inputs to obtain the portion of design space that minimized the process footprint, cost and achieved PI targets while respecting process constraints. ${ }^{17,18,42,43} \mathrm{~A}$ nonlinear programming (NLP)-based formulation was considered to perform direct and inverse input-output mapping calculations considering the design inputs (physical dimensions) instead of the operational inputs of the system.

Bottom-up approaches have been proposed to systematically find promising designs that employ $\mathrm{PI}$ and SM concepts. Transport phenomena and reactions were viewed as building blocks that were rearranged in a superstructure-based optimization. The optimization either minimized a measure of cost, footprint, or consumption of raw materials and resources, or maximized yield, efficiency, profit, etc. ${ }^{44-51}$ Nevertheless, process operability has only been incorporated into reported studies as complementary analyses that were limited to flexibility and safety targets. The presence of a comprehensive process operability framework could benefit such approaches by exploring a wider range of achievability and controllability aspects with appropriate sensitivity to provide design adjustments. 
In this dissertation, process operability methods are proposed to incorporate both design and operational inputs to evaluate and rank competitive modular designs. Specific operational challenges of modular and intensified energy systems are identified and addressed by a new methodology that estimates the $\mathrm{Ol}$ in terms of subregions using computational geometry tools. A multilayer framework is developed to systematically analyze the achievability of $\mathrm{PI}$ targets in a modular design region (MDR) containing candidate designs for SM and PI. The OI in terms of subregions and the multilayer framework are presented in Chapter 4 and Chapter 5, respectively.

\subsection{Modular Systems Costing Challenges}

The deployment of commercial modular technologies presents a variety of challenges such as assuring feasible and profitable operations. Process modularization is often achieved with higher degrees of customization. The application of conventional TEA methods is hindered because modular units present extremely low processing capacities in comparison with typical large-scale plants. These units also present integrated process topologies that differ from the one operation per unit scheme that is employed for the cost of large-scale plants. ${ }^{41}$

Process integration and intensification might generate multifunctional modular units that stand out from their conventional counterparts in terms of efficiency and footprint. Moreover, drastic changes for heat integration and reaction rate improvement are likely to affect utilities generation and management. Consequently, comparative economic evaluation of highly integrated modular plants may only be possible in a plantwide manner, for example, by scaling total costs with respect to a certain chemical product for direct comparison to traditional plants.

Additional cost challenges derive from the economy of scale. Traditional largescale plant designs rely on the economy of scale, in which larger productions decrease the relative cost of production. Modular systems are disadvantaged by economies of scale because more construction material is required for smaller production volumes proportionally. 
Modular units are expected to be standardized and mass produced, unlike conventional-scale plants. As more modular units are manufactured, unit costs may decrease due to improvement in both individual skills and organizational routines. Firstof-a-kind designs are expected to have higher costs, whereas $\mathrm{n}^{\text {th }}$-of-a-kind designs are presumably cheaper due to accrued know-how (or learning-by-doing) and manufacturing experience. Technological maturity is achieved when the costs to manufacture considered pieces of equipment steadily approach constant values. Notably, these reductions in cost are not spontaneous and must be managed. ${ }^{52}$

Historically, experience curve techniques have been applied to describe the effect of the economy of learning on the purchase cost of the most diverse technologies, ranging from cars to chemical plants. ${ }^{52-54}$ In the scope of manufacturing modular units in Chemical Engineering, the incorporation of the learning aspects have been sparsely studied. ${ }^{55-57}$ Moreover, the inclusion of economy of learning in the TEA of modular systems that are also intensified is yet to be addressed.

The consideration of economy of learning in modular manufacturing has potential to support the economic evaluation of more realistic scenarios. Nevertheless, requirements associated to cost data and experience curve parameters may prevent fast and effective techno-economic and profitability analyses. Particularly, capital and operating costs are commonly obtained from undisclosed price quotations, which are only conceivable to plant designs closer to completion. The economy of learning widely varies and depends on diverse factors such as research investment, market trends, process specifications, etc. ${ }^{52}$

In this dissertation, a systematic TEA framework is proposed for costing intensified modular systems. Conventional costing techniques are extended to allow estimation of capital and operating costs of modular units. Economy of learning concepts are included to consider the effect of experience curves on purchase cost. Profitability measures are scaled with respect to production of a chemical of interest for comparison with plants of traditional scale. In the developed framework, a base case scenario is analyzed to identify the relevance of the economy of learning and cost parameters that are yet to be established for modular projects that will be deployed. Then, a sensitivity analysis step is 
conducted to define changes in relevant variables that benefit the construction of modular systems. In the final step, scenarios in which the modular technology presents breakeven and further reductions in cost are identified. Chapter 7 explores the modular TEA framework and its application to a newly synthesized modular hydrogen unit.

\subsection{Previous Nonlinear Programming-based Operability Approaches}

A previously developed NLP-based operability approach extended process operability for

the design of emerging energy systems. ${ }^{18,43}$ The NLP-based operability approach has a solid foundation on calculations of the DIS. As an alternative to the analytical calculation of $M^{-1}$, an NLP-based optimization problem is formulated, aiming to obtain the elements in the AIS that can achieve a determined DOS. Originally created for square systems, this algorithm can also be applied to non-square systems with extra degrees of freedom. Previous applications contained optimization levels that were focused on obtaining an intensified input point associated with modular dimensions and an optimal nominal operation. Nevertheless, the robust calculations of $M^{-1}$ are versatile and suitable for other operability purposes.

The concepts of feasible DIS (DIS*) and feasible DOS (DOS*) were introduced to tackle situations in which not all the desired output points are achievable. The DIS* elements are mapped to DOS* elements, and these sets are the outcome of the optimization formulation. The DOS* is the closest set in terms of distance to the initially defined DOS; and the DIS* is the set with the correspondent input elements, obtained through mapping of the DIS*, as $D I S^{*}=M^{-1}\left(D_{O S}^{*}\right)$.

The employed optimization formulation initially considers a DOS given by output ranges. This DOS is discretized, generating a set of desired output elements. Then, an error minimization problem is posed, in which the objective function is formulated to minimize the distance between the feasible and the desired output elements. Using the notation from Equation (1), this minimization problem is described as P1 in Equation (24) below for each $\mathrm{j}^{\text {th }}$ element of the discretized DOS, $y_{j} \in$ DOS. 


$$
\text { P1: } \quad \Phi_{j}=\underset{u_{j}^{*}}{\operatorname{minimize}}\left(\rho\left(y_{j}, y_{j}^{*}\right)\right)
$$

\section{Subject to}

Nonlinear model (Equation (1))

$$
\begin{aligned}
& u^{\text {min }} \leq u_{j}^{*} \leq u^{\text {max }} \\
& c_{1}\left(u_{j}^{*}\right) \leq 0 \\
& c_{2}\left(u_{j}^{*}\right)=0
\end{aligned}
$$

where $y_{j}$ is an element of the discretized DOS; $y_{j}^{*}$ is an element of DOS ${ }^{*} ; u_{j}^{*}$ is an element of DIS*; $M\left(u_{j}^{*}\right)=y_{j}^{*}$ where $M$ is the nonlinear process model defined above; $\rho$ is the relative distance function between $y_{j} \in D O S$ and $y_{j}^{*} \in D O S^{*} ; u^{\min }$ and $u^{\text {max }}$ are the lower and upper inputs bounds, respectively; $c_{1}$ is an optional set of linear and nonlinear inequality constraints; and $c_{2}$ is an optional set of linear and nonlinear equality constraints. Note that the lower and upper input bounds can be set outside of the AIS limits to obtain feasible elements outside of the original available limits. Here, the following form of distance function $\rho$ is considered:

$$
\rho\left(y_{j}, y_{j}^{*}\right)=\sum_{i=1}^{p}\left(\left(y_{i, j}-y_{i, j}^{*}\right) / y_{i, j}\right)^{2}
$$

where the subscript $i$ indicates the coordinates of $y_{j}=\left(y_{i, j}\right)_{i \in\{1,2, \ldots, p\}} \in D O S$ and $y_{j}^{*}=$ $\left(y_{i, j}^{*}\right)_{i \in\{1,2, \ldots, p\}} \in D^{*} S^{*}$. Note that other metrics could be applied to define this distance, such as absolute value and other vector norms.

After the feasible sets are obtained, another optimization level can be applied. Employing DIS* and DOS*, the problem P2 is formulated to attain a determined target (for instance, performance, cost, PI targets, environmental targets, and so on) defined in Equation (26), as follows: 


$$
\begin{gathered}
\text { P2: } \quad \Omega=\underset{u^{*}}{\operatorname{maximize}}\left(\varphi\left(u^{*}, y^{*}\right)\right) \\
\text { Subject to } \\
u^{*} \in D_{I} S^{*} \\
y^{*} \in D^{*} S^{*}
\end{gathered}
$$

where $u^{*}$ and $y^{*}$ are elements of the $\mathrm{DIS}^{*}$ and $\mathrm{DOS}^{*}$, respectively; and $\varphi\left(u^{*}, y^{*}\right)$ is a generic objective function that can represent any of the aforementioned targets. For many cases, this optimization level is translated to a selection of elements from $\mathrm{P} 1$, allowing an easy inclusion of bound constraints and linear or nonlinear constraints.

Updated developments for this approach consisted of simultaneously solving the problems P1 and P2 by elaborating a bilevel optimization formulation that combines the inverse model calculation and the attainment of desired targets in tandem. Parallel programming techniques have also been added to the bilevel formulation to increase the speed of nonlinear calculations. For details on these developments see ref. ${ }^{18}$.

In this dissertation, both $\mathrm{P} 1$ and $\mathrm{P} 2$ are generalized to receive any process model and system dimensionality. The codes are written in MATLAB with the embedded nonlinear solver fminsearch. Then, the NLP-based approach is included in the introduced Operability App. Chapter 6 describes this effort, including a non-square application that has 7 inputs and 3 outputs.

\subsection{Illustration of Process Operability Concepts: Classic Shower Problem}

The shower problem is a toy problem that has been proposed to illustrate the principles of steady-state operability analysis. ${ }^{2,9}$ Cold and hot water streams are mixed, producing a final flow and temperature. The stream flows are controlled by two valves, or shower knobs. The goal of the shower problem is to change the amount of hot and cold water to obtain a desired temperature and flow. Therefore, cold and hot flowrates are MVs, and the final temperature and total flow are CVs. The nonlinear process model for this problem is described by 


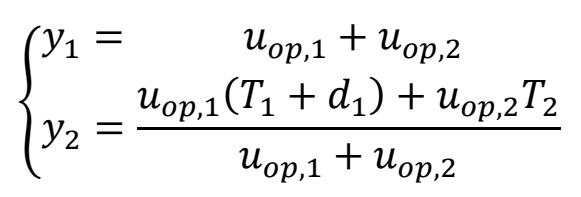

where $u_{o p, 1}$ and $u_{o p, 2}$ are the MVS of cold and hot flowrates, respectively; $y_{1}$ and $y_{2}$ are the CVs of final flowrate and temperature, respectively; $T_{1}$ and $T_{2}$ are the temperatures of the cold and hot water, respectively. In this example, a disturbance in the cold-water temperature is considered. This disturbance is represented as an absolute temperature change in $T_{1}$ and corresponds to the variable $d_{1}$.

Note that using the model representation from Equation (1) as a reference, there are no state variables or process constraints in this example. The shower problem is formulated as a system with 2 operational inputs, 2 outputs and 2 disturbances, yielding $m_{o p}=p=2$ and $q=1$. Unlike typical chemical systems, this nonlinear model is simple and can be inverted analytically.

The AISop, EDS and DOS are readily obtained with the following assumptions: (i) the shower knobs can be turned to provide cold and hot flows within the ranges $0 \leq$ $u_{o p, 1} \leq 4 \mathrm{gal} / \mathrm{min}$ and $0 \leq u_{o p, 2} \leq 3 \mathrm{gal} / \mathrm{min}$, respectively; (ii) the cold flow temperature has fluctuation within the range of $-10 \leq d_{1} \leq+10$, where $d_{1}^{N}=0$ indicates no disturbance perturbation; (iii) the ideal shower operation is at $y_{1}^{N}=5 \mathrm{gal} / \mathrm{min}$ and $y_{2}^{N}=$ $84^{\circ} \mathrm{F}$; and (v) operations within the ranges of $3 \leq y_{1} \leq 7 \mathrm{gal} / \mathrm{min}$ and $74 \leq y_{2} \leq 94^{\circ} \mathrm{F}$ are acceptable.

The AOS can be obtained using the process model from Equation (15). Cold and hot temperatures of $T_{1}=60^{\circ} \mathrm{F}$ and $T_{2}=120^{\circ} \mathrm{F}$, respectively, are considered. The three types of $\mathrm{Ol}$ are calculated in this example. All sets and calculations are represented by their boundaries, or frontiers. The process model is inverted analytically and $T_{1}$ and $T_{2}$ are substituted by their values, resulting in the following equation:

$$
\left\{\begin{array}{l}
u_{2, o p}=\frac{y_{1}\left(y_{2}-60-d_{1}\right)}{\left(60-d_{1}\right)} \\
u_{1, o p}=y_{1}-u_{o p, 2}
\end{array}\right.
$$

In this example, the s-OI is used to measure the system ability to achieve a DOS defined by the acceptable ranges above and no disturbance change. The set AISop is 
defined by operating ranges of hot and cold flow. The is EDS defined as a single point. The DOS is defined by desired ranges of final temperature and total flowrate. These sets AIS op, EDS and DOS are described as follows:

$$
\begin{gathered}
A I S_{o p}=\left\{u_{o p} \in \mathbb{R}^{2} \mid(0,0) \leq u_{o p} \leq(4,3)\right\} \\
E D S=d_{1}^{N}=0 \\
D O S=\left\{y \in \mathbb{R}^{2} \mid(3,74) \leq u_{o p} \leq(7,94)\right\}
\end{gathered}
$$

The sets $A_{\text {op }} \cap D I S_{\text {op }}$ and AOSNDOS are obtained for the calculation of s-OI through input and output perspectives, respectively. In MATLAB, the AOS is obtained by simulation the process model using the sets AIS op and EDS defined above. Similarly, the DISop is calculated using the inverse model and the sets EDS and DOS. The sets AISop, DISop, AOS and DOS are converted into two-dimensional polygons. The operations of intersection are carried out using Boolean routines embedded in MATLAB. Finally, areas of $A I S_{o p} \cap D I S_{o p}$, DIS op, AOS $\cap$ DOS and DOS are estimated and the s-OI is obtained using Equations (9) and (10). Figure 1 contains the representation of the operability sets, including intersections and obtained values of s-OI. The values of s-OI lower than $100 \%$ indicate that are certain setpoints inside the DOS that aren't achievable.
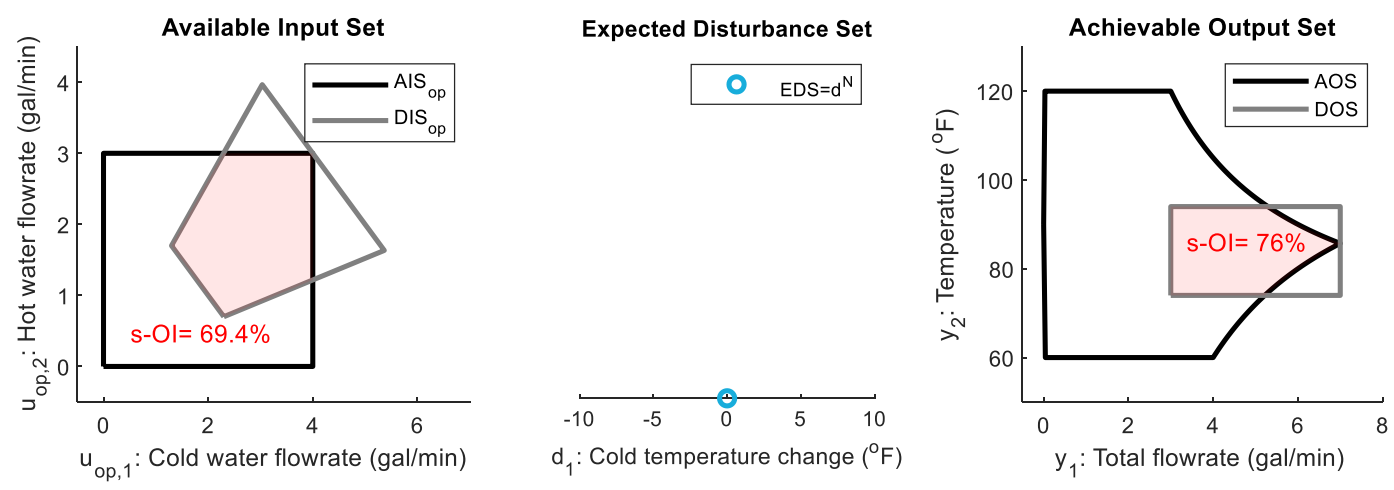

Figure 1. Calculations of s-Ol for the shower problem.

The next task is the estimation of $\mathrm{r}$-OI. The $\mathrm{r}$-Ol measures how the manipulated variables can be changed to keep the operation at $y^{N}$ given expected disturbances in the cold flow temperature. Here, the DOS is only one operating point, and the EDS assumes expected ranges of $-10 \leq d_{1} \leq+10$. The EDS is evenly divided into 5 case scenarios, and these sets are represented as follows: 


$$
\begin{gathered}
E D S=\{-10,-5,0,+5,+10\} \\
D O S=y^{N}=(5,84)
\end{gathered}
$$

For $d \in E D S$, an $\operatorname{AOS}(d)$ is calculated using the predefined AISop and the attributed disturbance value. Similarly, $D I S_{o p}(d)$ sets are calculated using $y^{N}=(5,84)$ and attributed disturbances values. Figure 2 depicts the obtained operability sets. The disturbance in cold flow temperature shifts the achievable regions and the required combinations of MVs to keep the system at the nominal point $y^{N}$.

The DISop and AOS are then computed as a union and intersection of each disturbance scenario, respectively. The obtained DISop represents required MV combinations to reject all expected disturbances. The AOS corresponds to the feasible region that is always achievable with respect to the presence of expected disturbances. Figure 3 shows the obtained operability sets. Note that the DIS op is entirely inside AISop, and $y^{N}$ is inside the portions of AOS that are achievable for all $d \in E D S$, indicating that the shower knobs can always be adjusted to regulate amounts of hot and cold stream and keep the outputs at $y^{N}$.
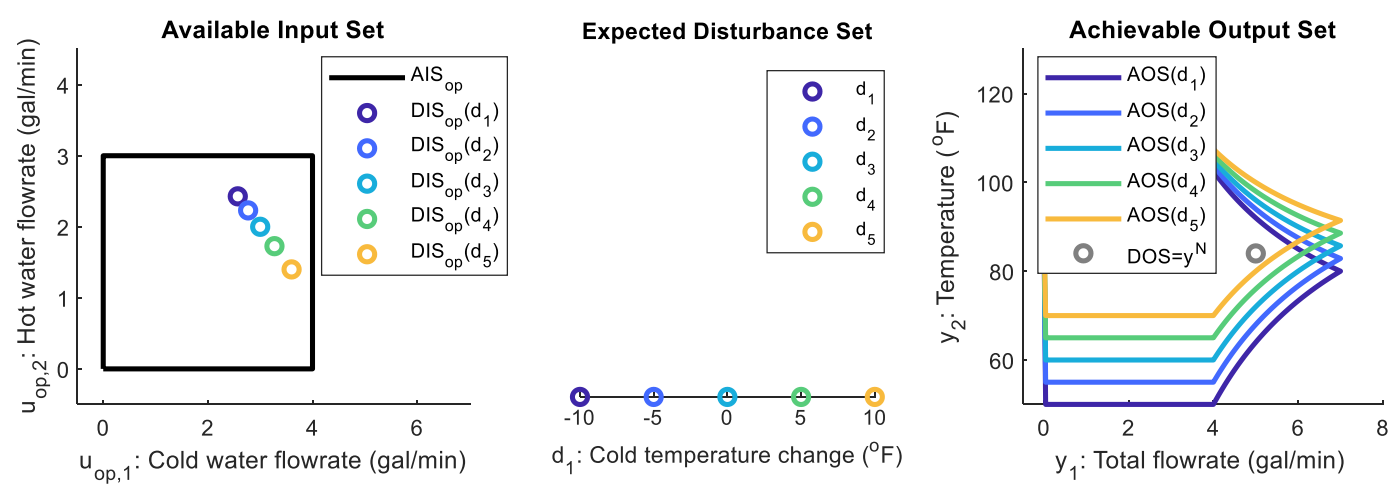

Figure 2. System behavior with respect to regulatory action. 

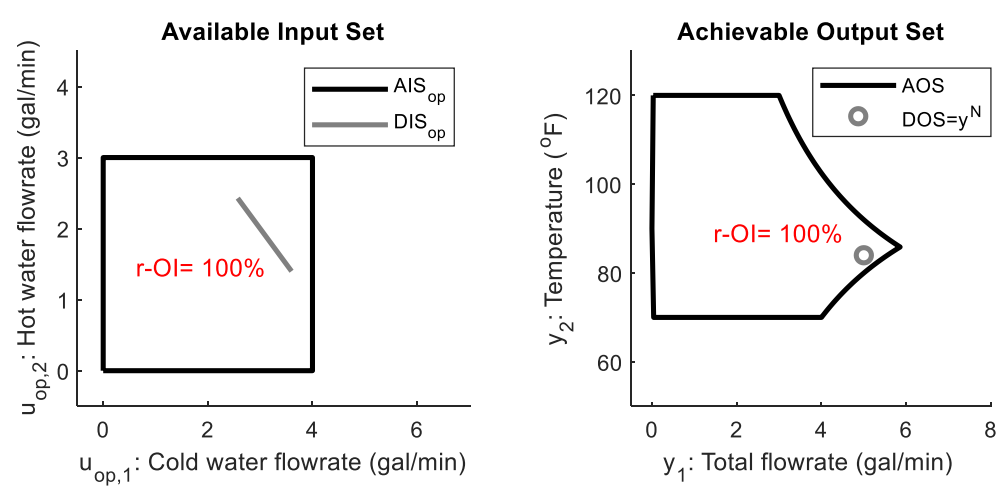

Figure 3. Calculations of $\mathrm{r}-\mathrm{Ol}$ for the shower problem.

The last task is the calculation of o-OI. The o-Ol quantifies how the system rejects disturbances at every operating point inside the acceptable ranges for $y_{1}$ and $y_{2}$. Therefore, both DOS and EDS are given by ranges, corresponding to Equations (19) and (20). The same procedure applied for $\mathrm{r}$-OI calculation is repeated, but the entire DOS is employed instead of a single point. Figure 4 contains the obtained operability sets. Although the AOS is shifted exactly like the previous case, the DOS is now enlarged. Every $D I S_{o p}(d)$ in turn contains more MV combinations, corresponding to requirements to achieve all the setpoints of the DOS at each disturbance scenario.

The DISop and AOS are computed in the same fashion. Figure 5 depicts the obtained operability sets. Note that the DISop has a bigger size and isn't completely inside

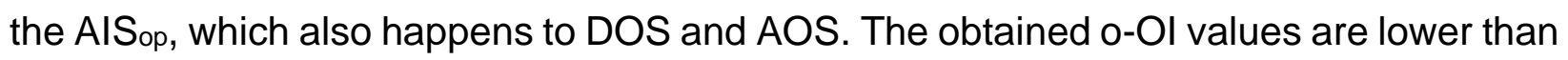
$100 \%$, indicating that there are disturbances in which no movement of the shower knobs can achieve all the setpoints contained in the DOS.
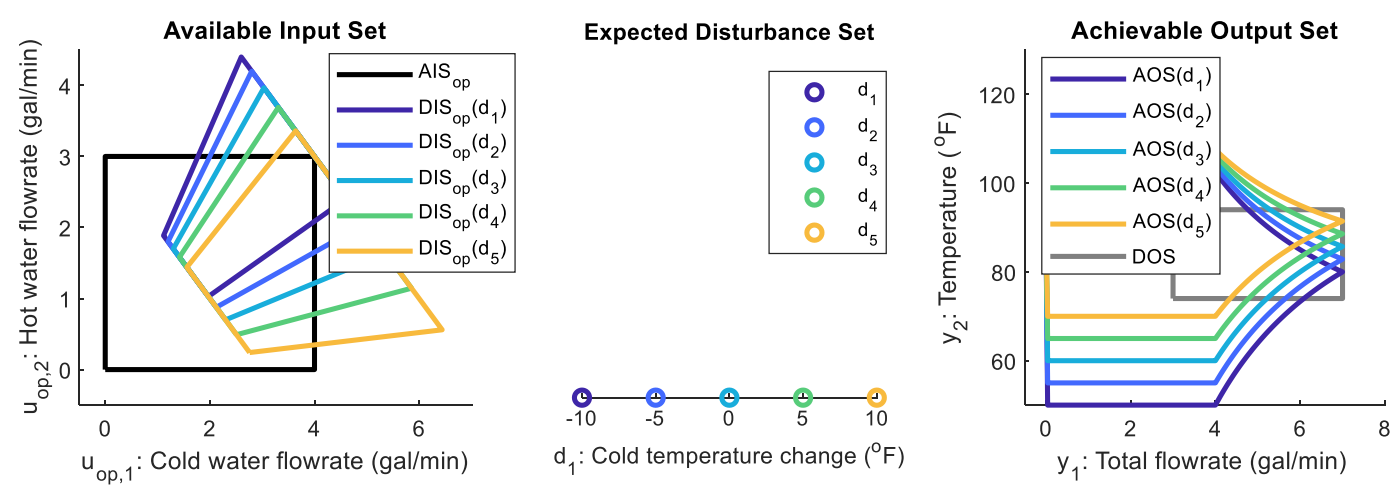

Figure 4. System behavior with respect to a collection of regulatory actions. 

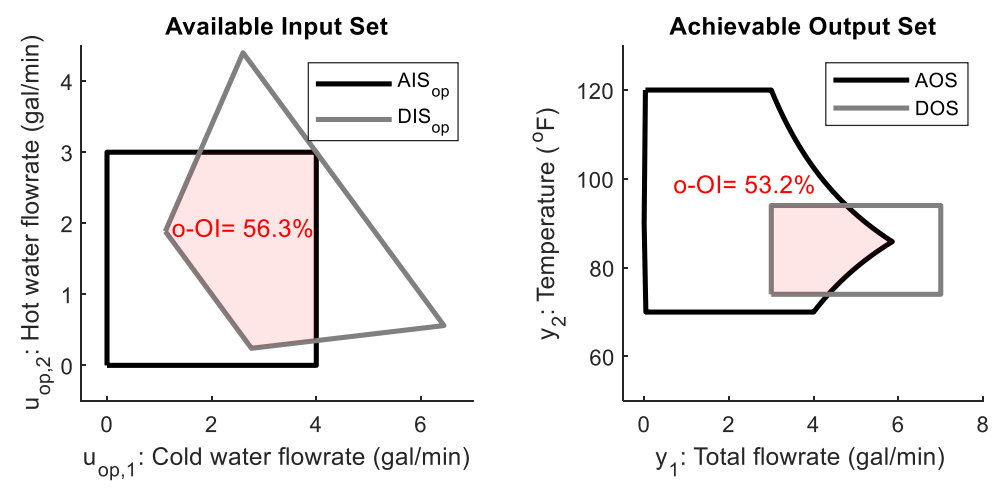

Figure 5. Calculations of o-Ol for the shower problem.

Note that when $\mathrm{r}-\mathrm{Ol}$ and $\mathrm{o}-\mathrm{Ol}$ are calculated, the operations of union and intersection that form DISop and AOS, respectively, eliminate specific information regarding which disturbances are compensated. Another possible drawback is that if $A O S(d) \cap D O S=\varnothing$ for some $d \in E D S$, i.e., if any disturbance makes the DOS unachievable, the r-OI or o-Ol are $0 \%$ when evaluated using Equations (10) and (11). Alternative calculations of $\mathrm{r}$-OI and o-Ol presented in Chapter 4 inform which disturbances and setpoints are not compensated and achieved. In this dissertation, the shower problem is used to illustrate portions of the introduced developments. 


\section{Chapter 3 Multimodel Representation}

The multimodel approach applies space discretization techniques to represent the originally developed nonlinear input-output mapping with multiple linearized models. These models are structured as connected input-output polytopes, facilitating the computation of space manipulations, intersections, and hypervolumes. The inverse model and other calculations are performed in terms of polytopes and barycentric interpolations, resulting in reduced computational time efforts in comparison to NLPbased operability approaches. ${ }^{4}$ In this chapter, a multimodel representation that describes the process operability sets is developed. Then, techniques that gradually increase space resolution to obtain $M^{-1}$ and regions that attain SM and PI objectives are introduced.

\subsection{Multimodel Representation Concepts}

First, the multimodel representation is developed for systems without disturbances. Then, the EDS is appended to both input and output sets to include the presence of disturbances in the representation. Finally, two geometrical representations of the nonlinear system are analyzed, one that divides inputs and outputs and another that depicts all sets as part of the same multidimensional space.

\subsubsection{Input-Output Representation without Disturbances}

The generation of a set of polytopes is the initial task in obtaining the multimodel representation. Elements of either the AIS or AOS can be used to generate these polytopes. The expected geometrical shapes of these sets indicate that the AIS is better suited for this task. As described in Equation (2), the AIS is usually created from ranges, which most of the time allows this set to be evenly decomposed into smaller subsets. Conversely, the AOS has an unpredictable shape due to potential model nonlinearities, and its decomposition into finer subsets may not always be applicable for systematic methods of space discretization.

Then, to obtain the multimodel representation, mesh and triangulation techniques are applied to the AIS elements, generating a set of input polytopes. Since each input 
point has an output counterpart, corresponding output polytopes are also generated. Each $\mathrm{k}^{\text {th }}$ pair of connected polytopes is represented as follows:

$$
\begin{aligned}
& P_{k}^{u}=\left\{u_{1, k}, u_{2, k}, \ldots, u_{j, k}, \ldots, u_{J, k}\right\} \\
& P_{k}^{y}=\left\{y_{1, k}, y_{2, k}, \ldots, y_{j, k}, \ldots, y_{J, k}\right\}
\end{aligned}
$$

where $P_{k}^{u}$ and $P_{k}^{y}$ are input and output connected polytopes; $u_{j, k} \in \mathbb{R}^{m}$ and $y_{j, k} \in \mathbb{R}^{p}$ are the vertices of the input and output polytopes, respectively; $J$ is the total number of vertices; the subscript $j$ is associated with index of elements; and the subscript $k$ is associated with the action of numbering the obtained paired polytopes.

Here, if triangulation techniques are applied after evenly dividing the AIS, the grid elements will be divided into polytopes with $m+1$ vertices, holding the property of always being convex. If $p=m$, the property of convexity will also hold for the output polytopes, while for $p \neq m$, the same cannot be inferred. Note that overlaps among obtained output polytopes are likely to happen and must be considered for calculations of hypervolume and intersections.

Assuming a total number of $K$ polytopes, the AIS and AOS can be represented as follows:

$$
\begin{gathered}
A I S=\left\{P_{k}^{u} \mid k \in S\right\} \\
A O S=\left\{P_{k}^{y} \mid k \in S\right\}
\end{gathered}
$$

where $S=\{1,2, \ldots k, \ldots, K\}$ is the set with counts for each connected polytope.

Figure 6 shows an example of this representation for a generic $2 \times 2$ system containing 6 input-output data points. In this example, $S=\{1,2,3,4\}$ has $K=4$ polytopes that are simplices in two dimensions, i.e., triangles. Each pair of triangles links 3 inputoutput points, in which the edges correspond to 3 linear models. 

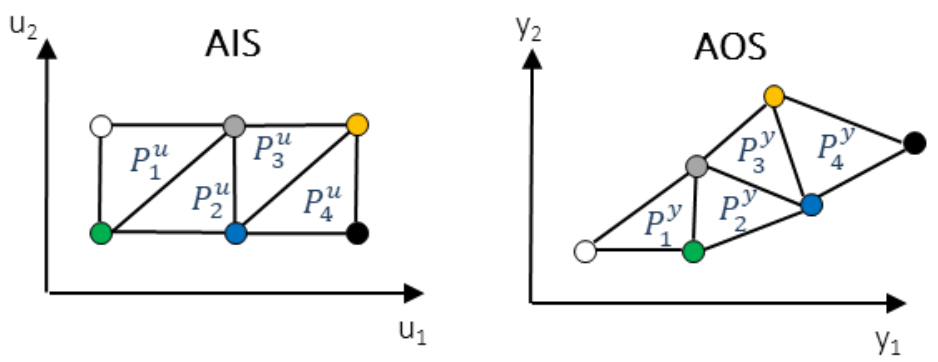

Figure 6. Illustration of the multi-model polytope representation for a $2 \times 2$ system.

Some advantages of employing the multimodel representation for $M$ are the easy quantification of the $\mathrm{Ol}$ and the straightforward model inversion $\mathrm{M}^{-1}$ using computational geometry tools. In particular, the calculations of $M^{-1}$ are performed for a region of the AOS by selecting the output polytopes inside the region and then verifying the corresponding paired polytopes in the AIS. Still using the example above, suppose one verifies that $\operatorname{DOS} \cap A O S=\left\{P_{3}^{y}, P_{4}^{y}\right\}$; therefore $M^{-1}[D O S \cap A O S]=\left\{P_{3}^{u}, P_{4}^{u}\right\}$, associated with 2 triangles in the input space. If needed, interpolations can be applied to obtain $M^{-1}$ in terms of the points inside the triangles. Such multimodel representation can be produced from space discretization techniques such as the division of the set as a grid and Delaunay triangulation.

\subsubsection{Inclusion of Disturbances}

When disturbances are present and evaluations of $\mathrm{r}-\mathrm{Ol}$ or $\mathrm{o}-\mathrm{Ol}$ are desired, the multimodel representation is extended to include disturbances. The EDS is appended to the AIS, AOS and DOS, resulting in the Cartesian products $A I S^{\prime}=A I S \times E D S, A O S^{\prime}=$ $A O S \times E D S$, and $D O S^{\prime}=D O S \times E D S$, respectively. This representation augments the AISAOS mapping, describing the system behavior for every disturbance scenario. The new sets are defined as follows:

$$
\begin{aligned}
& A I S^{\prime}=\left\{(u, d) \in \mathbb{R}^{m+q} \mid u \in A I S \text { and } d \in E D S\right\} \\
& A O S^{\prime}=\left\{(y, d) \in \mathbb{R}^{p+q} \mid y \in A O S \text { and } d \in E D S\right\} \\
& D O S^{\prime}=\left\{(y, d) \in \mathbb{R}^{p+q} \mid y \in \text { DOS and } d \in E D S\right\}
\end{aligned}
$$


The multimodel representation can also be obtained by evenly dividing AIS' into a grid and performing Delaunay triangulation. A representation similar to that of Equations (22) and (23) is the outcome with each $\mathrm{k}^{\text {th }}$ pair of connected polytopes given by

$$
\begin{aligned}
P_{k}^{u, d} & =\left\{(u, d)_{1, k},(u, d)_{2, k}, \ldots,(u, d)_{j, k}, \ldots,(u, d)_{J, k}\right\} \\
P_{k}^{y, d} & =\left\{(y, d)_{1, k},(y, d)_{2, k}, \ldots,(y, d)_{j, k}, \ldots,(y, d)_{J, k}\right\}
\end{aligned}
$$

where $P_{k}^{u, d}$ and $P_{k}^{y, d}$ are input and output connected polytopes; $(u, d)_{j, k} \in \mathbb{R}^{m+q}$ and $(y, d)_{j, k} \in \mathbb{R}^{p+q}$ are the vertices of the new input-output spaces; and $J, j$ and $k$ follow the same convention as Subsection 3.1.1. Equivalently, for a total number of $K$ polytopes, the AIS' and AOS' can be represented as follows:

$$
\begin{aligned}
& A I S^{\prime}=\left\{P_{k}^{u, d} \mid k \in S\right\} \\
& A O S^{\prime}=\left\{P_{k}^{y, d} \mid k \in S\right\}
\end{aligned}
$$

where $S=\{1,2, \ldots k, \ldots, K\}$ is the set with counts for each connected polytope.

The system representation using AIS' and AOS' allows computations of r-OI and o-Ol using direct mappings only. The verification of necessary DIS is substituted by goals related to achievement of DOS'. Chapter 4 explains how calculations of r-OI and o-Ol are performed using this extended multimodel representation.

\subsubsection{Joint System Representation}

The above representation of the nonlinear system as connected polytopes allows the calculations of diverse measures of OI. Inputs, outputs, and disturbances are separated and interpreted as disjoint polytopes. Alternatively, a joint representation of the nonlinear system can be useful for other tasks employed in the design of emerging systems. One case is the selection of design or operating regions that are highly constrained.

More aggressive process integration strategies create process constraints that involve input, output, and disturbance variables indistinctively. For example, a constraint associated with an energy balance can depend on inlets, outlets and heat exchange 
driven by environment temperatures. Following the same notation, the AIS and AOS are given by

$$
A I S \times A O S=\left\{(u, y) \in \mathbb{R}^{m+p} \mid u \in A I S \text { and } y \in A O S\right\}
$$

Each $\mathrm{k}^{\text {th }}$ pair of connected polytopes is substituted by a single $\mathrm{k}^{\text {th }}$ polytopes.as follows:

$$
P_{k}=\left\{(u, y)_{1, k},(u, y)_{2, k}, \ldots,(u, y)_{j, k}, \ldots,(u, y)_{J, k}\right\}
$$

For a total number of $K$ polytopes, the AIS $\times A O S$ set can be represented as follows:

$$
A I S \times A O S=\left\{P_{k} \mid k \in S\right\}
$$

where $S=\{1,2, \ldots k, \ldots, K\}$ is the set with counts of all the polytopes.

Figure 7 shows an example of a system with 2 operational inputs and 1 output represented by both joint and disjoint representations.

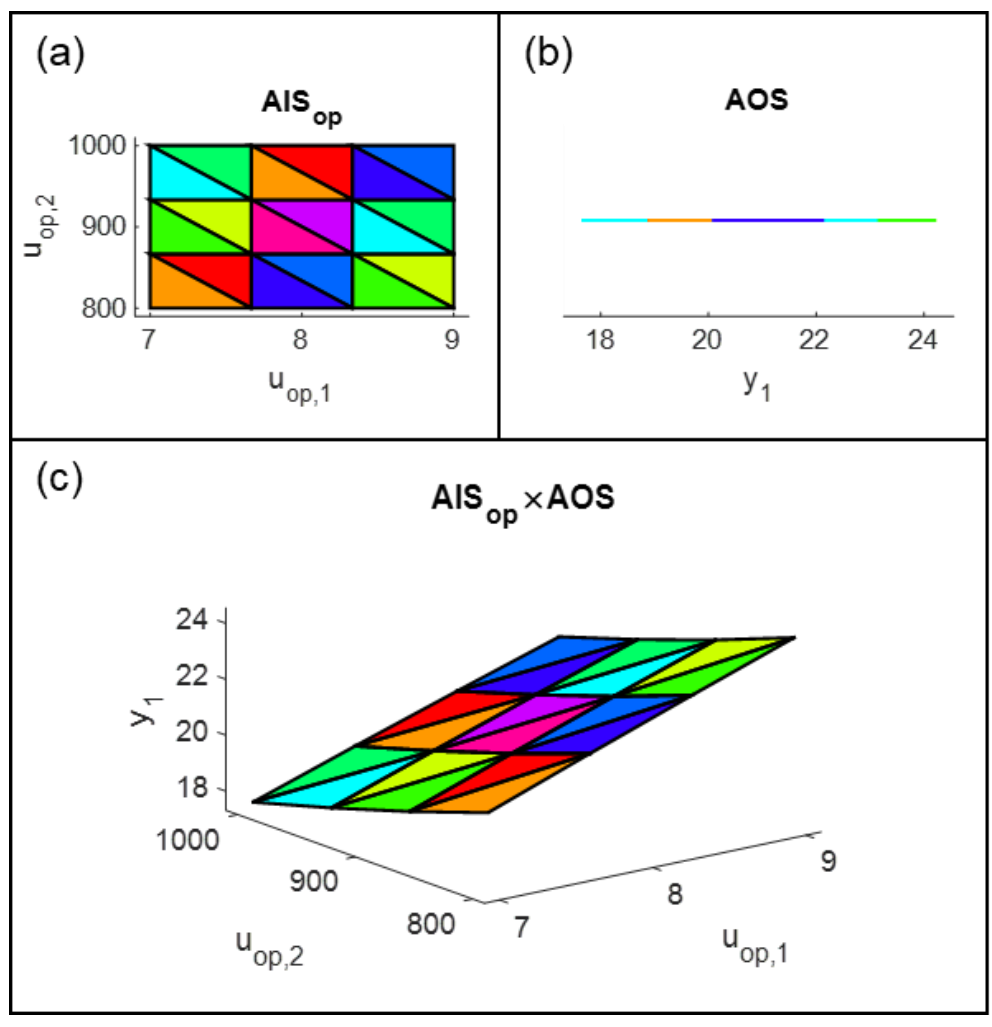

Figure 7. 2x1 example: (a) disjoint system representation and (b) joint system representation. 
In this example, 18 polytopes represent the nonlinear system. The disjoint representation generates 18 triangles in the input space, which are connected to 18 lines in the output space. The joint representation is a three-dimensional surface in which the AIS op is shifted according to AOS values. Joint representations with more than 3 dimensions can only be easily represented mathematically. In Subsection 3.2.2, joint representations are employed with space selection methods regarding feasible regions.

\subsection{Methods for Space Selection}

The multimodel representation can be readily used for calculations of Ol. The resolution of the space is defined by the amount of divisions imposed in the input space (AIS or AIS') before the triangulation step. Usually, the space resolution can be low for the goal of ranking design according to the operational performance if differences between designs are evidenced. However, improved definitions of the operability regions may be required for the task of model inversion, especially if regions in the AIS des are analyzed. A detailed multimodel representation of the entire input-output would require an extensive amount of function evaluations, demanding higher computational expenses. As a solution, an iterative algorithm is developed to locally increase the number of models as the nonlinear system achieves desired regions. This subsection introduces methods for model inversion based on computational geometry calculations and mixed-integer linear programming (MILP) concepts.

\subsubsection{Inverse Multimodel Calculation}

The model inversion is calculated using the multimodel representation. An iterative algorithm is formulated to increase the number of models that achieve a given DOS. The input-output mapping is represented by connected polytopes, and a refinement step takes place at each iteration. The outcome is a multimodel representation that is fine at regions that achieve the desired operation.

The AIS is initially evenly halved to generate $2^{m}$ m-dimensional parallelepipeds. Then, the intersection AOS $\cap$ DOS is calculated using the multimodel representation from 
Equations (24) and (25). Every output polytope in $P_{k}^{y}$ undergoes the operation $P_{k}^{y} \cap D O S$. The output polytopes that provide $P_{k}^{y} \cap D O S \neq \varnothing$ are classified into two groups: $S^{\prime}=$ $\left\{k \subseteq S \mid P_{k}^{y} \cap D O S \neq \emptyset\right.$ and $\left.P_{k}^{y} \cap \operatorname{Fr}(D O S) \neq \emptyset\right\}$ and $S^{\prime \prime}=\left\{k \subseteq S \mid P_{k}^{y} \cap D O S \neq \emptyset\right.$ and $P_{k}^{y} \cap$ $\operatorname{Fr}(D O S)=\emptyset\}$, in which $\operatorname{Fr}(D O S)$ is the frontier of the DOS. The iteration ends by selecting parts of $S$ to be reserved or refined. $S^{\prime}$ indicates polytopes in the border of the DOS, which are further divided. $S^{\prime \prime}$ and $S-\left(S^{\prime} \cup S^{\prime \prime}\right)$ indicates polytopes that are completely in the interior and exterior of the DOS, respectively, and therefore are not refined.

The halving operation is repeated using inputs polytopes $\left\{P_{k}^{u} \mid k \in S^{\prime}\right\}$. In the new iteration, $S^{\prime}$ is defined as $S$ and the procedure further defines the output polytopes that achieve $\operatorname{Fr}(D O S)$. This procedure is repeated until a predefined number of divisions is obtained. Note that as the operability sets are defined in the real space, the iterations would go on indefinitely without a desired resolution.

Figure 8 shows the application of the inverse multimodel algorithm to the classic shower problem. In Figure 8a, the procedure is applied without triangulations. In Figure $8 b$, triangulations are incorporated as well as a final interpolation step. The interpolation is only performed with triangulation because of convexity of the obtained polytopes. The result of a triangulation procedure is always a simplex: a shape with $n+1$ vertices for an n-dimensional space. For example, in two-dimensional spaces, triangulations generate triangles; in three-dimensional spaces, tetrahedra; and so on.

The developed algorithm is designed to work with higher-dimensional cases, and a two-dimensional example is chosen for illustrative purposes. The inverse multimodel calculation is recommended for situations in which a higher definition of AIS $\cap$ DIS is desired. For comparison of designs using Ol values, the inverse multimodel is not recommended because a higher definition of the input-output mapping is not required to evidence difference among evaluated designs. In Chapter 7 , the inverse multimodel is used for selection of nominal design and operation of a modular hydrogen unit application. 


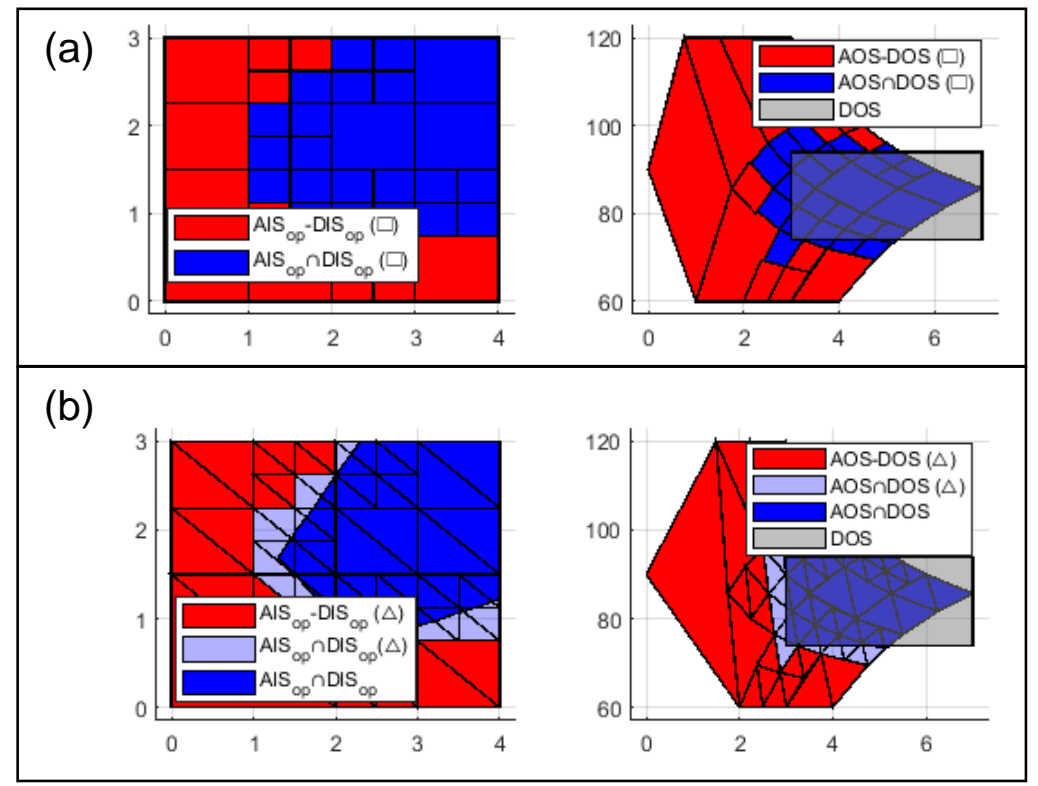

Figure 8. Inverse multimodel application to the shower problem (servo case): (a) without triangulations and (b) with triangulations and interpolations.

\subsubsection{Iterative Mixed Integer Linear Programming-based Algorithm}

Here, the inverse multimodel iterative algorithm is extended to direct the intersections towards the minimization of an objective function. The inclusion of additional aims to the inverse problem is motivated by the presence of design input variables. While a larger AISop usually represents more freedom in terms of how MVs adjust process performance, it may be preferable to focus on specific portions of the AIS des that achieve desired operation, design targets and process constraints.

The joint system representation is employed to characterize the nonlinear model in a single multidimensional space. The AIS des and DOS are transformed into a set of linear inequality constraints. Additional process constraints are incorporated in terms of input and output variables. The goal of achieving the DOS is substituted by the goal of achieving a feasible region obtained from all linear constraints. The feasible region is converted to a polytope of dimension $m_{\text {des }}+n$ using computational geometry techniques.

Applications related to SM and PI motivate the creation of an objective function that minimizes process footprint, while the achievement of PI targets is presented as part 
of the desired operation. The inverse multimodel calculation is then adapted to include the objective associated with footprint minimization. The calculations are performed using linear objective functions and process constraints as part of a MILP formulation. Nonlinear objective functions and constraints must also be linearized if present. The developed iterative MILP-based algorithm is explained as follows:

Starting with the entire AISdes set, a solution is generated in each iteration, so that the input space is gradually narrowed around the optimal solution. When there is no change in the solution from one iteration to the next, the algorithm achieves convergence and the optimal design region is obtained. Triangulation tools are also employed for the calculation of intersections involving the set of obtained geometrical entities that represent the linearized models. ${ }^{58}$ The algorithm is developed to accommodate a generic number of dimensions. Figure 9 shows a schematic representation of the developed algorithm with all the employed steps.

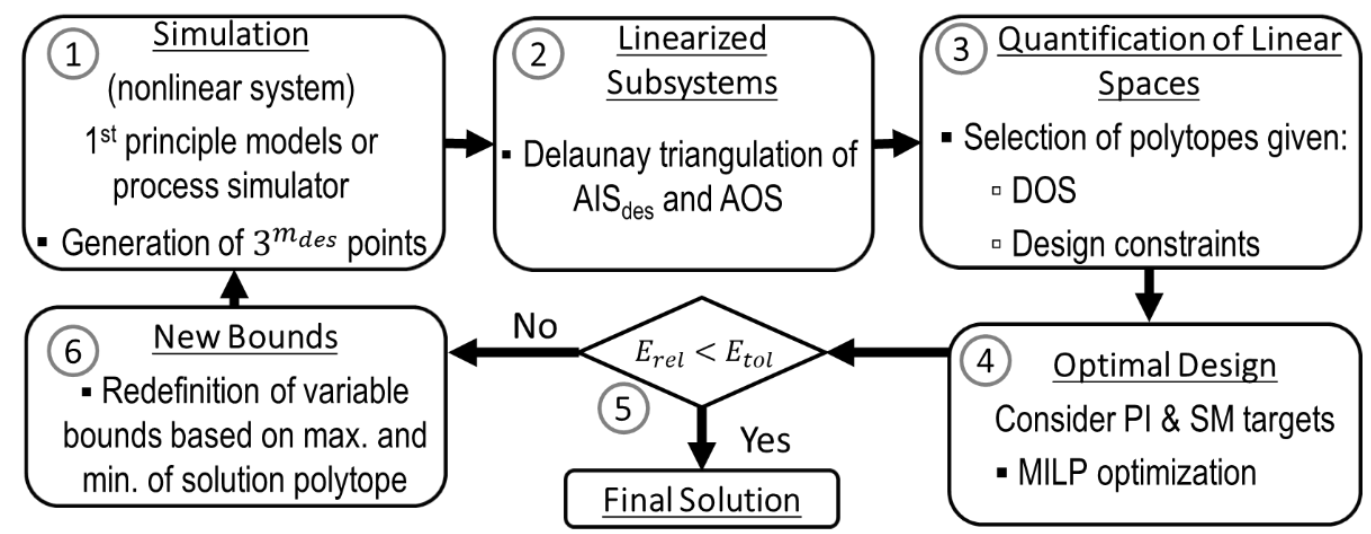

Figure 9. Steps of the MILP-based iterative approach.

Each of the enumerated steps in Figure 9 is briefly discussed below.

(1) Simulation: According to the energy system application of interest, $3^{m_{\text {des }}}$ data points are generated using either first-principle models or a process simulator, in which $m_{d e s}$ is the dimensionality of the analyzed square system (2-D, 3-D, etc.). This number of data points is chosen to obtain a relatively low number of function evaluations of the process model $M$ in each iteration and thus maintain a low computational expense. Alternatively, a less coarse initial grid could be adopted in the initial iterations for a more detailed 
representation of the nonlinear system with the expense of a higher computational time. As the available inputs are known, the input set is evenly divided into a grid and the corresponding output points can be obtained through simulation. This uniform grid division is adopted here as it provides a lower computational time for the task of finding approximated optimal points in step 4. A nonuniform division of the grid would be recommended for the task of further characterizing the representation of AOS $\cap$ DOS or $A I S \cap D I S$ as shown in Subsection 3.2.1. The outcome of this step is a set of input-output data points.

(2) Linearized Subsystems: Using the obtained input-output data points, the spatial discretization technique of Delaunay triangulation is applied to build the joint multimodel representation. This triangulation is performed using the obtained input points, and appending output points, as described in Subsection 3.1.3. The technique generates a set of $K$ paired polytopes that is indexed by $S=\{1,2, \ldots, K\}$. Each polytope, $P_{k}$, represents a set of linear models from the input to the output space. The set $S$ is used to indicate all the $K$ paired polytopes, i.e., $\left\{P_{k} \mid k \in S\right\}$.

(3) Quantification of Linear Spaces: The obtained polytopes are analyzed according to achievability of the DOS and process constraints. The feasible region described by a polytope of dimension $m_{\text {des }}+n$ is used to evaluate which portions of the nonlinear model fulfill the DOS and process constraints. Each $P_{k}$ that provides $P_{k} \cap$ Feasible Region $\neq \varnothing$ is selected. The outcome of this step is a set $S^{\prime} \subseteq S$ of $K^{\prime}$ paired polytopes that satisfy the criteria for AIS des, DOS, and process constraints.

(4) Optimal Design: In order to obtain an optimal design point, an MILP minimization problem is formulated for the selection of the polytope from $S^{\prime}$ that gives the optimal inputoutput coordinates considering PI, SM and process constraints. Weights associated with vertices of the polytopes and barycentric interpolations allow this optimal solution to be inside one of the considered polytopes. In addition to the weights, a binary variable is assigned to each pair of polytopes so that the MILP solver only selects one polytope as a solution. The MILP-minimization problem can be mathematically represented as follows: 


$$
\begin{aligned}
& \chi=\underset{\text { minimize }}{\chi}\left(\varphi_{\text {linear }}\right) \\
& \qquad u_{d e s}, b_{k} \\
& \text { Subject to } \\
& u_{d e s} \in A I S_{d e s}, y \in D O S \\
& b_{k} \in\{0,1\}^{K^{\prime}} \\
& \sum_{\text {PI and SM targets }} b_{k}=1 \\
& \text { Process constraints }
\end{aligned}
$$

where $\varphi_{\text {linear }}$ is a linear or linearized objective function (process footprint for SM) and $b_{k}$ is the binary variable assigned to each polytope $P_{k}$ from $\left\{P_{k} \mid k \in S^{\prime}\right\}$. The MILPminimization problem results in a selected polytope $P_{s o l}$, inside which is the obtained optimal solution. The optimal solution corresponds to one input-output point calculated from barycentric interpolations using the vertices of $P_{s o l}$. This point is therefore associated with an input $u_{\text {des }} \in A I S_{\text {des }}$, mapped to some $y \in A O S$. The MATLAB subroutine intlinprog is used here to solve the formulated problem with tolerances for both integers and constraints of 0.001 . Note that although the selection of $P_{k}$ is performed in the joint space of dimension $m_{\text {des }}+p$, the MILP formulation is elaborated considering only the input space as decision variables. Therefore, interpolations are allowed for convex polytopes with $m_{\text {des }}+1$ vertices. Additional details of this formulation can be found along with applications in Subsections 3.2.3 and 5.2.

(5) Stopping Criteria: Using the solution for the optimal design of the current iteration and the solution of the previous iteration, the relative difference between solutions is calculated, $E_{r e l}$. If this difference is smaller than a predefined threshold or tolerance, $E_{t o l}$, the algorithm converges, and the final solution is obtained (Final Solution). Otherwise, new variable bounds are defined based on the polytope of the current solution (step 6).

(6) New Bounds: If the algorithm has not converged, new input bounds are chosen based on the selected polytope from step $4, P_{s o l}$. These bounds are described by the set $\left\{u_{\text {des }, i, P_{s o l}}^{\min } \leq u_{i} \leq u_{\text {des,i,P }}^{\max } \mid 1 \leq i \leq m_{\text {des }}\right\}$, in which $u_{d e s, i, P_{s o l}}^{\min }$ and $u_{d e s, i, P_{s o l}}^{\max }$ are the minimum and maximum values that characterize the polytope of the solution in the inputs. For each input dimension $i$ (up to $m_{d e s}$ ), the new minimum and maximum bounds are calculated as follows: 


$$
\begin{aligned}
& u_{i, P_{\text {sol }}}^{\min }=\min \left(\left\{u_{\text {des }, i, j \text { Psol }} \mid 1 \leq j \leq m_{\text {des }}+1\right\}\right) \\
& u_{i, P_{\text {sol }}}^{\max }=\max \left(\left\{u_{\text {des }, i, j \text { Psol }} \mid 1 \leq j \leq m_{\text {des }}+1\right\}\right)
\end{aligned}
$$

where $j$ refers to each vertex of the polytope of the solution $P_{s o l} . P_{s o l}$ has $m_{\text {des }}+1$ vertices because the joint multimodel representation obtained using triangulation is performed in the input space. Once the new bounds are defined, the algorithm goes back to step 1 for the next iteration.

\subsubsection{DMA-MR Application}

By converting methane to hydrogen fuel and value-added chemical benzene, the DMA$M R$ is a candidate energy system for the modular utilization of NG. The modularization of this system potentially benefits the on-site exploitation of the shale gas formations in remote locations, eliminating the need for expensive pipelines and elaborate industrial infrastructure usually present in conventional large-scale processes. Here, PI and SM are enabled by the combination of reaction and separation in the membrane reactor. This process integration strategy promotes enhanced reactivity by shifting the reaction equilibrium towards the products, inducing footprint reduction by combining the two unit operations.

For operability applications, previously developed work addressed the DMA-MR modeling from experimental data considering the non-oxidative conversion of methane as a two-step reaction mechanism. ${ }^{59,60}$ Catalysis and membrane transport studies were employed to obtain adequate reaction kinetics that were suitable to membrane reactor models constituted by a set of ordinary differential equations (ODEs). ${ }^{61}$ Figure 10 below shows a schematic of the co-current configuration of the DMA-MR. 


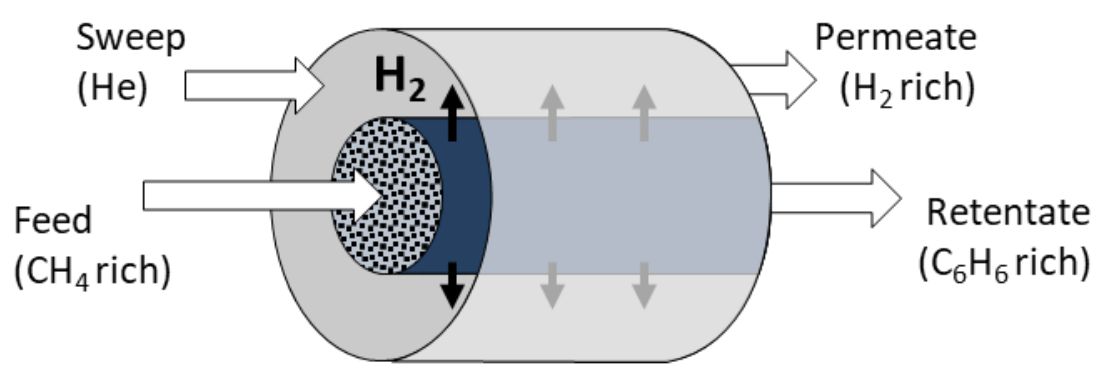

Catalyst $\square$ Membrane

Figure 10. Co-current DMA-MR schematic.

The following two-step reaction mechanism is considered for the DMA-MR:

$$
\begin{array}{ll}
2 \mathrm{CH}_{4} \rightleftharpoons \mathrm{C}_{2} \mathrm{H}_{4}+2 \mathrm{H}_{2} & r_{1}=k_{1} C_{\mathrm{CH}_{4}}-k_{1}^{\prime} \frac{C_{C_{2} \mathrm{H}_{4} C_{H_{2}}^{2}}}{C_{C H_{4}}} \\
3 \mathrm{C}_{2} \mathrm{H}_{4} \rightleftharpoons \mathrm{C}_{6} \mathrm{H}_{6}+3 \mathrm{H}_{2} & r_{2}=k_{2} C_{C_{2} \mathrm{H}_{4}}-k_{2}^{\prime} \frac{C_{C_{6} H_{6} C_{H_{2}}^{3}}}{C_{C_{2} \mathrm{H}_{4}}^{2}}
\end{array}
$$

where $C$ stands for species concentrations; $k_{1}$ and $k_{2}$ are the reactions rate constants for the first and second steps, respectively; and the superscript ' indicates inverse reaction rate constant. The generated ODE set from molar balances in the tube and shell can be found in ref. ${ }^{17}$.

The input and output spaces of this system are prescreened for selecting available design inputs and other operability sets. This activity consists of the primary study of the system behavior. The outcome is the selection of the sets AIS and AOS and the determination of the DOS. A total of 8 inputs and 4 outputs is considered for the DMAMR applications presented in this dissertation. Tables 2 and 3 show the design and operational input variables and the corresponding available ranges, respectively. Table 4 shows the output variables and the corresponding desired ranges. Each of the studied subsystems have input and output sets of distinct dimensionalities composed by variables and ranges presented in Tables 2, 3, and 4. 
Table 2. Design input variables and available ranges.

\begin{tabular}{|c|c|}
\hline design input variable & available range \\
\hline Reactor length $(\mathrm{cm})$ & $10-100$ \\
\hline Tube diameter (cm) & $0.5-2.0$ \\
\hline Selectivity (-) & $300-1 \times 10^{5}$ \\
\hline Permeance $\left(\mathrm{mol} /\left(\mathrm{s} \cdot \mathrm{m}^{2} \mathrm{~atm}^{1 / 4}\right)\right)$ & $1 \times 10^{-4}-1 \times 10^{-2}$ \\
\hline
\end{tabular}

Table 3. Operational input variables and available ranges.

\begin{tabular}{ll}
\hline operational input variable & available range \\
\hline \hline Methane feed $\left(\mathrm{cm}^{3} / \mathrm{min}\right)$ & $7-9$ \\
Temperature $\left({ }^{\circ} \mathrm{C}\right)$ & $800-1000$ \\
Sweep gas feed $\left(\mathrm{cm}^{3} / \mathrm{min}\right)$ & $9-11$ \\
Tube pressure $(\mathrm{atm})$ & $1.00-1.12$ \\
\hline
\end{tabular}

Table 4. Output variables and desired ranges.

\begin{tabular}{ll}
\hline output variable & desired range \\
\hline \hline Benzene production $(\mathrm{mg} / \mathrm{h})$ & $20-25$ \\
Methane conversion $(\%)$ & $35-45$ \\
Hydrogen production $(\mathrm{mg} / \mathrm{h})$ & $3-6$ \\
Cost factor $(-)$ & $0-100$ \\
\hline
\end{tabular}

For the modularization of the reactor, the minimization of process footprint and achievement of $\mathrm{PI}$ targets while respecting process constraints are considered. This task is translated to an optimization formulation that is inserted in both NLP and MILP-based algorithms. The nonlinear objective function of process footprint, $f$, is described by the sum of membrane area and reactor volume as follows: 


$$
f(L, D)=\pi D L+\frac{\pi}{4} D^{2}
$$

where $L$ is the reactor length and $D$ is the tube diameter. The nonlinear objective function must be linearized for application of the MILP-based iterative algorithm. The linearization is described in the application of step (4) of the algorithm below, and results in Equation (46).

A minimal benzene production of $20 \mathrm{mg} / \mathrm{h}$ is considered as a process intensification target. One of the employed process constraints is the $L / D$ ratio that assures plug-flow operation, ${ }^{62}$ also given as a function of reactor length and tube diameter as follows:

$$
\frac{L}{D} \geq 30
$$

When needed, simulations of this system are carried out in MATLAB. The embedded stiff ODE solver, ode15s is employed. The choice of solver is justified by the nature of the ODE system, which is likely to require small step sizes due to permeation of species through the membrane.

Here, a subsystem of lower dimensionality demonstrates the effectiveness of the iterative MILP-based algorithm. The subsystem is defined by two-dimensional design input and output spaces. The input and output variables are selected from Tables 2 and 4 and are structured as follows:

$$
\begin{aligned}
& u_{d e s, 1} \stackrel{\text { def }}{=} \text { Reactor length }(\mathrm{cm}) \\
& u_{d e s, 2} \stackrel{\text { def }}{=} \text { Tube diameter }(\mathrm{cm}) \\
& y_{1} \stackrel{\text { def }}{=} \text { Benzene production }(\mathrm{mg} / \mathrm{h}) \\
& y_{2} \stackrel{\text { def }}{=} \text { Methane conversion }(\%)
\end{aligned}
$$

The application of each of the steps of the iterative algorithm introduced in Subsection 3.2.2 is explained below.

(1) Simulation: Here, in the first iteration, the input bounds $u_{d e s, 1}=\left[\begin{array}{ll}10 & 100\end{array}\right]$ and $u_{d e s, 2}=$ $\left[\begin{array}{ll}0.5 & 2.0\end{array}\right]$ are evenly divided into $3^{2}=9$ input points. The output points are obtained through simulation of the nonlinear system, using the process model $M$. In Figure 11a, it is possible to see the formed grid for each iteration. In Figure 12a, the obtained output for 
each iteration is depicted. The four-dimensional joint multimodel representation is visually illustrated by two separate plots, resembling the disjoint representation.

(2) Linearized Subsystems: The obtained input-output points are used as inputs for this step, generating the joint multimodel representation. Figures 11a and 12a depict the set of polytopes for each iteration, indicated by $S=\{1,2, \ldots, 8\}$.

(3) Quantification of Linear Spaces: Using the obtained multimodel representation from step 2 , the polytopes that satisfy the feasible region defined by input constraints $(L / D \geq$ 30 ) and operability sets are selected, generating a subset $S^{\prime} \subseteq S$. Since the intensification target $y_{1} \geq 20 \mathrm{mg} / \mathrm{h}$ is already incorporated into the DOS, it isn't separately included to define the feasible region. Figures $11 \mathrm{~b}$ and $12 \mathrm{~b}$ show the regions associated with the input and output constraints, respectively.

(4) Optimal Design: The MILP formulation is then originated using the selected polytopes $\left\{P_{k} \mid k \in S^{\prime}\right\}$. The following minimization problem is formulated:

$$
\begin{aligned}
& \chi=\underset{w_{j k}, b_{k}}{\chi} \operatorname{minimize}\left(h\left(u_{d e s, 1}, u_{d e s, 2}\right)\right) \\
& \text { Subject to } \\
& 10 \leq u_{d e s, 1} \leq 100 \\
& 0.5 \leq u_{d e s, 2} \leq 2 \\
& 20 \leq y_{1} \leq 25 \\
& 35 \leq y_{2} \leq 45 \\
& u_{d e s, 1} / u_{d e s, 2} \geq 30 \\
& b_{k} \in\{0,1\}, k \in S^{\prime} \\
& \sum_{k=1}^{K^{\prime}} b_{k}=1 \\
& \sum_{j=1}^{3} w_{j k}=b_{k}, k \in S^{\prime} \\
& 0 \leq w_{j k} \leq 1, \quad k \in S^{\prime}, \quad j \in\{1,2,3\} \\
& u_{i}=\sum_{k=1}^{K^{\prime}}\left(\sum_{j=1}^{3} u_{d e s, i, j k} \cdot w_{j k}\right), \quad i \in\{1,2\} \\
& y_{i}=\sum_{k=1}^{K^{\prime}}\left(\sum_{j=1}^{3} y_{i, j k} \cdot w_{j k}\right), \quad i \in\{1,2\}
\end{aligned}
$$


where $b_{k}$ is the binary variable assigned to the polytope $P_{k}, k \in S^{\prime} ; w_{j k}$ is the weight of a vertex $j$ of $P_{k} ; u_{d e s, i, j k}$ and $y_{i, j k}$ are the input-output data points of polytope $P_{k}$. The footprint, initially given by the sum of total membrane area and reactor volume, is approximated here by a linearization around the nominal reactor length and tube diameter as follows:

$$
\begin{aligned}
& h\left(u_{\text {des }, 1}, u_{\text {des }, 2}\right)=\left(D_{o}+0.25 D_{0}^{2}\right) u_{\text {des }, 1}+\left(L_{0}+0.5 L_{0} D_{0}\right) u_{d e s, 2} \\
& h\left(u_{d e s, 1}, u_{d e s, 2}\right)=0.5625 u_{d e s, 1}+12.5 u_{d e s, 2}
\end{aligned}
$$

where $L_{0}=10 \mathrm{~cm}$ and $D_{0}=0.5 \mathrm{~cm}$ are the nominal values of reactor length and tube diameter, respectively.

Figures $11 \mathrm{~b}$ and $12 \mathrm{~b}$ show the selection of a triangle in each iteration, for inputs and outputs, respectively, as well as the calculation of the optimal points (plotted inside the figures).

(5) Stopping Criteria: A tolerance error of $1 \%$ with respect to each variable for both input and output coordinates is considered for convergence to the optimal solution.

(6) New Bounds: Smaller regions of the AIS des are considered as the algorithm approaches convergence. Figure 11a shows these regions being reduced until convergence is achieved.

The algorithm converges to an optimal solution in 5 iterations, resulting in a DMAMR with reactor length of $17.05 \mathrm{~cm}$ and tube diameter of $0.57 \mathrm{~cm}$ corresponding to a benzene production of $20 \mathrm{mg} / \mathrm{h}$ and methane conversion of $35 \%$. The total computational time of the algorithm is 6 seconds. This results in an optimal design point. The iterative MILP-based algorithm can also generate optimal design regions instead of optimal points. Chapter 5 includes the iterative MILP-based algorithm as part of a multilayer framework in which optimal design regions are employed. 
(a)

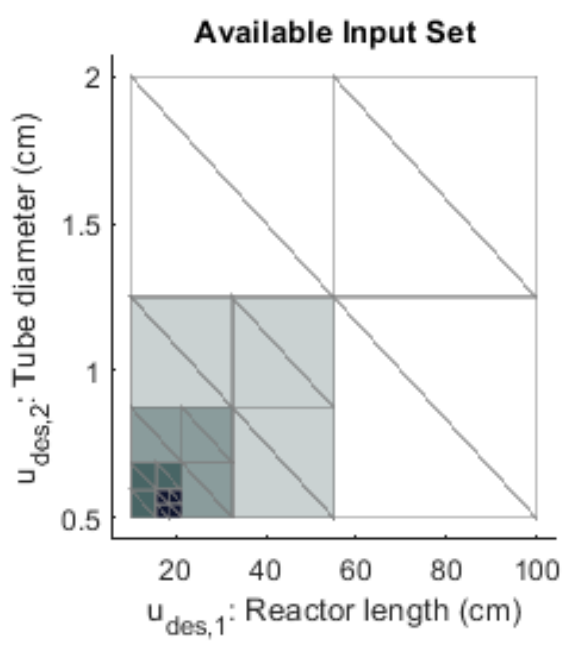

(b)

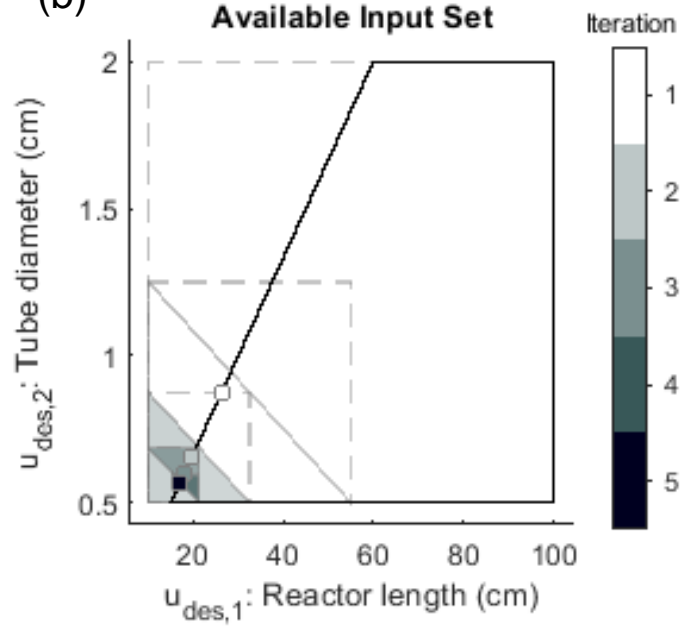

Figure 11. Two-dimensional case: (a) input variable bounds and triangulation; (b) selection of triangles and optimal solution points for each iteration.

(a)

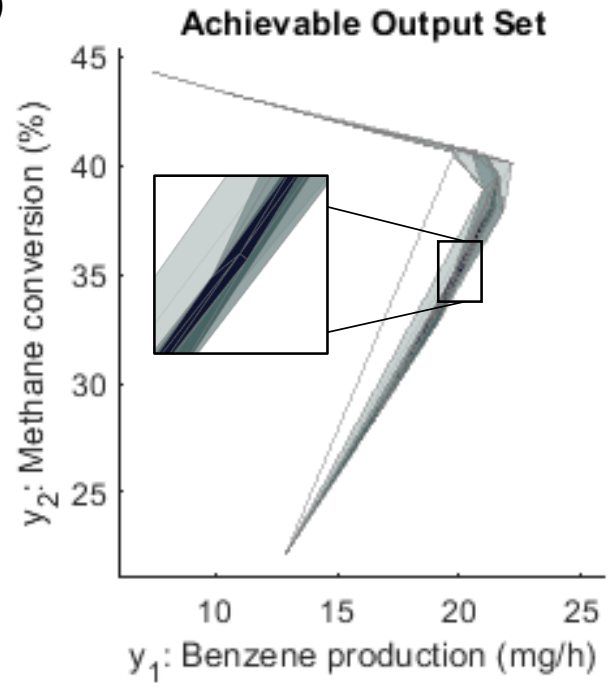

(b) Achievable Output Set Iteration

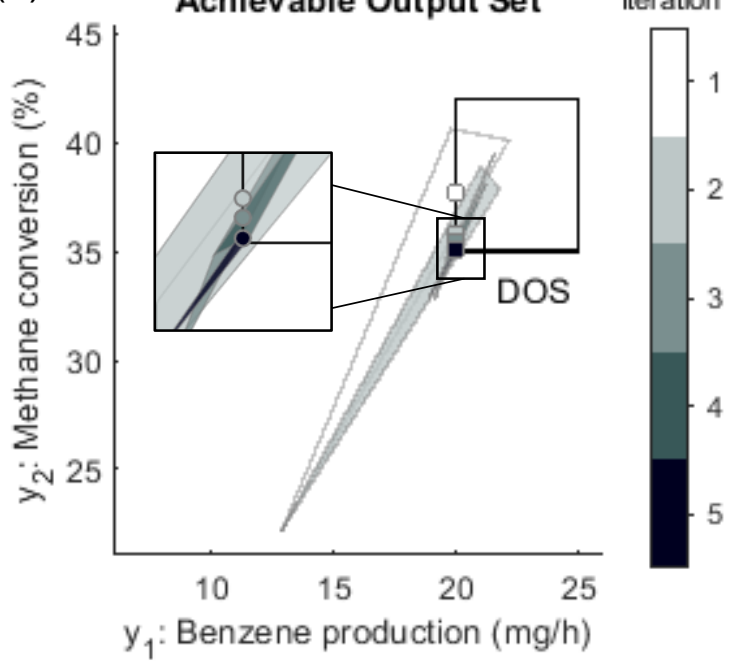

Figure 12. Two-dimensional case: (a) output mapping; (b) selection of triangles and optimal solution points for each iteration. 


\section{Chapter 4 Calculations of Operability Index}

The calculation of $\mathrm{Ol}$ is dependent on the metrics associated with length, volume, and hypervolume for spaces with more than three dimensions. These metrics can present challenges, especially for regions related to intersections between desired and available or achievable sets. A new measure of $\mathrm{OI}$ in terms of subregions is developed in this work to facilitate the quantification of OI. The actual hypervolume calculation is included as part of the multimodel operability approach. Alternative methods are proposed to estimate $r$ OI and o-OI without the inverse model or calculations of the AOS that is achievable with for all expected disturbances.

\subsection{Calculations with Hypervolumes and Subregions}

The multimodel approach described above originally employed the measure of $\mathrm{Ol}$ in terms of subregions. First, the DOS is evenly fragmented into a set of subregions. Then, the intersection $A O S \cap D O S$ is calculated using computational geometry tools. ${ }^{58} \mathrm{~A}$ subregion of the DOS is assumed to be achievable when it contains at least one point of the AOS in its interior. The ratio obtained between the number of achieved DOS subregions and the total number of DOS subregions defines this measure of OI. The bigger the value of the OI, the higher the achievability of the DOS. The number of divisions of the DOS is a parameter that can be changed to increase or decrease the number of subregions. For ranking competing designs, it is recommended to have enough divisions so that differences in the calculated values of OI can be detected. Possible limitations for 4 and higher-dimensional triangulations are overcome by adopting the MATLAB subroutine delaunayn, which corresponds to the generalized $\mathrm{n}$-dimensional triangulation.

The conventional measure of $\mathrm{Ol}$ is also incorporated into the multimodel approach. For such cases, the computational geometry tools are employed to obtain the hypervolumes of the sets $D O S$ and $A O S \cap D O S$. The volume of $A O S \cap D O S$ is calculated using the set complement $(D O S \backslash(A O S \cap D O S)=\{y \in D O S \mid y \notin A O S \cap D O S\})$ to overcome the possible presence of overlaps in the output polytopes. 
Both OI in terms of subregions and hypervolumes are tested for the classic shower problem. The disturbance is fixed at $d_{1}^{N}=0$. The AIS op is evenly divided into 100 elements and the disjoint multimodel representation is obtained with triangulation. The DOS is evenly divided into 100 elements for the calculation of Ol in terms of subregions, whereas, for hypervolumes, it is kept whole. Then, calculations of s-OI are performed. Figure 13 shows the AIS $\mathrm{op}, \mathrm{AOS}$, and both estimations of s-OI. Note that although the calculation with hypervolumes provides a more accurate result, the highlighted region in green presents nonconvexities which hinder higher-dimensional calculations. The calculation with subregions in turn analyzes achievability of the predetermined subregions in a way that nonconvexities of $A O S \cap D O S$ do not affect the estimation of the OI.

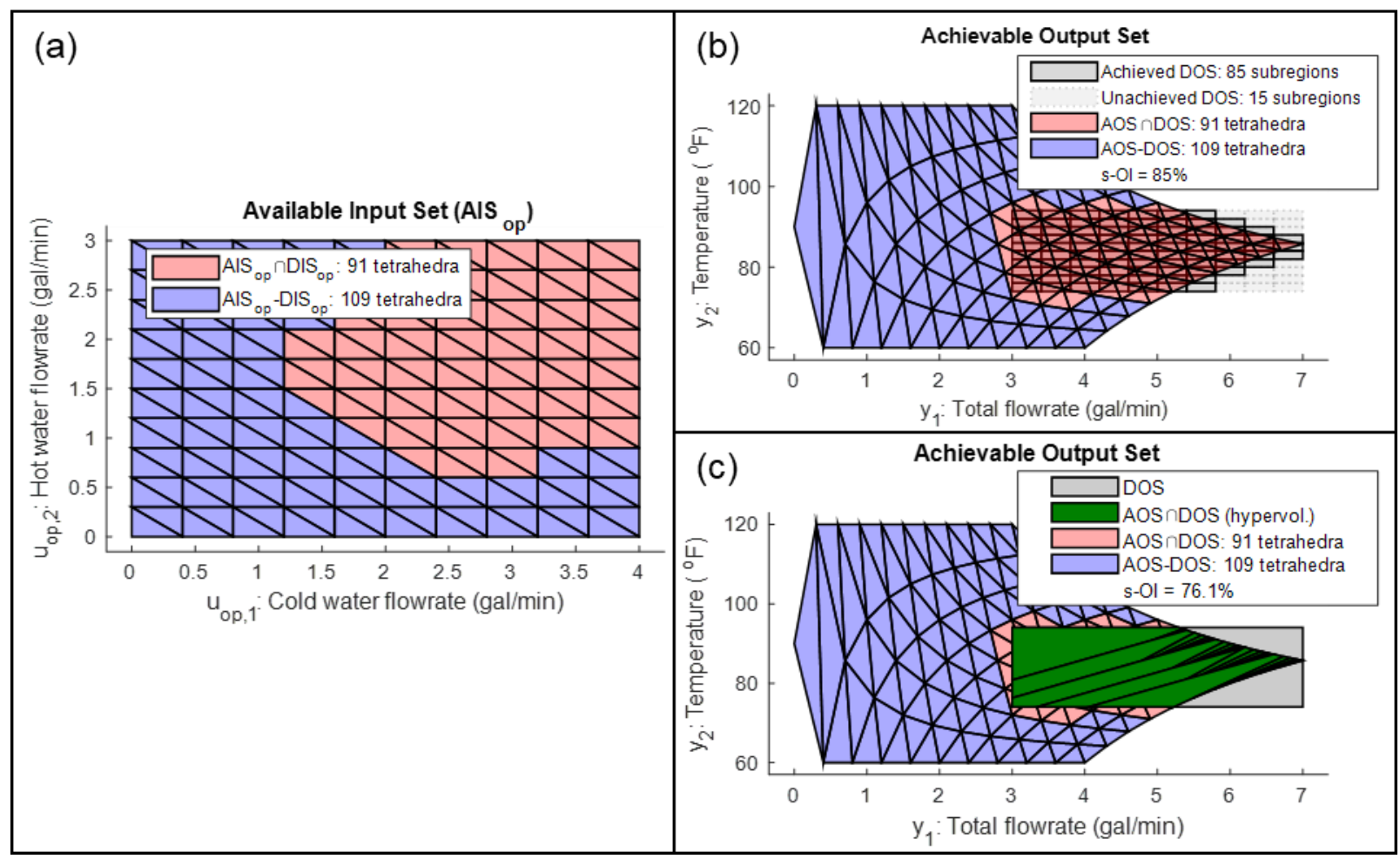

Figure 13. s-OI with multimodel representation for shower problem: (a) AIS; (b) AOS and s-OI subregions; (c) AOS and s-OI hypervolumes.

The quantification of $\mathrm{OI}$ is also challenged by systems in which the AOS shape does not present a measurable hypervolume. For example, a line in a two-dimensional output space, or a surface in a three-dimensional output space. For such cases, the hypervolume of the DOS tends to be many orders higher than that of $A O S \cap D O S$, resulting 
in a measure of Ol close to zero. Nevertheless, the nonlinear system might still be able to achieve certain regions of the DOS. This effect has been observed in modular and intensified energy system in which coupling effect occurs. ${ }^{1}$

In the calculation involving subregions, only one element $y \in A O S$ must be inside a given subregion for achievability. Therefore, cases in which the AOS has a low hypervolume but covers considerable extensions of the DOS can be addressed by the OI in terms of subregions. Occasional overlaps of AOS polytopes are thus ignored in this measure.

The significant difference between the two Ol measures is also demonstrated in a DMA-MR subsystem that contains three-dimensional operational inputs (MVs) and outputs (CVs). Figures 14a and 14b show the achievement of DOS in terms of subregions and hypervolumes, associated with maximum values of $\mathrm{s}-\mathrm{Ol}$ of $25 \%$ and $0.324 \%$, respectively. The AISop and differentiation between the sets $\left\{P_{k}^{y} \in A O S \mid P_{k} \cap D O S=\emptyset\right\}$ and $\left\{P_{k}^{y} \in A O S \mid P_{k} \cap D O S \neq \varnothing\right\}$ are omitted from this representation to facilitate visualization.

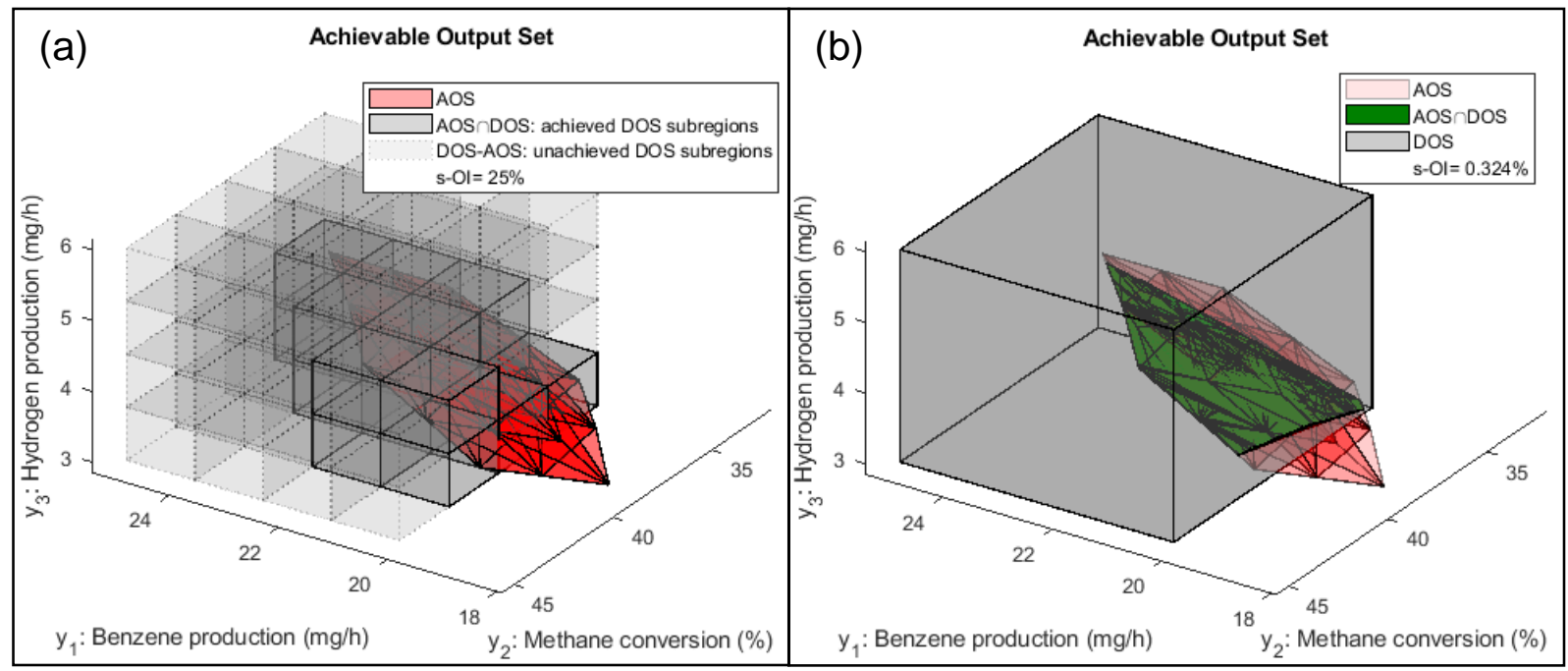

Figure 14. Calculation of output sets in terms of: (a) subregions; and (b) hypervolumes.

Note from Figures $14 \mathrm{a}$ and $14 \mathrm{~b}$ that the intersections $A O S \cap D O S$ are given by distinct regions. In Figure 14a, the intersection is represented by subregions in which the achieved subregions estimate $\mu(A O S \cap D O S)$, and all subregions estimate $\mu(D O S)$. In 
Figure $14 \mathrm{~b}$, the actual volumes of the intersection and DOS are employed. Visual comparison between the intersections supports the fact that it is easier to achieve portions of subregions of the DOS than portions of DOS in terms of actual volume.

Therefore, this DMA-MR subsystem is one example in which process integration results in an AOS that has almost no volume, even though it is generated in a threedimensional space. For this situation, the volumetric s-Ol may indicate a very small percentual achievability (less than 1\%). Nevertheless, as seen from the measure in terms of subregions, the AIS ${ }_{o p}$ can be enough to ensure achievability of portions of the DOS, being suitable for future analysis of set-point control.

A conclusion is that cases in which the AOS shape is too irregular are susceptible to difficulties in the measure of $\mathrm{s}-\mathrm{Ol}$ in terms of hypervolumes. The measure using subregions provides insights about achievability, being recommended for situations in which the AOS presents a strong nonlinear and/or nonconvex behavior, or cannot be visualized due to four or higher dimensionalities. Conversely, if the entire volume of the DOS is desired to be achieved, then the measure of Ol that employs hypervolumes is recommended.

Subsection 5.2 includes a detailed DMA-MR case study in which additional OI calculations are performed. The consequences of choosing different $\mathrm{OI}$ measures are analyzed in the scope of classifying and ranking modular designs. The conflict between Ol improvement and other objectives is also discussed.

\subsection{Inclusion of Disturbances in Operability Index Calculations}

The multimodel representation in Subsection 3.1.2 is considered here to represent the system behavior with respect to disturbance changes. The tasks of rejecting disturbances are conventionally quantified by input regions DISop and $A I S_{o p} \cap D I S_{o p}$. Here, disturbance rejection is quantified by achievement of DOS' using hypervolumes or subregions.

First, the readily available sets AIS, EDS and DOS are converted to AIS' and DOS' corresponding to Equations (26) and (28), respectively. Then, AIS' is evenly divided into 
a grid, and simulations are carried out to obtain the achievable outputs. The set AOS' is formed using achieved outputs and EDS, corresponding to Equation (27).

Triangulation is performed on AIS', generating $P_{k}^{u, d}$. The connections between elements of AIS' are extended to AOS', and $P_{k}^{y, d}$ is obtained. Finally, the same procedure from Subsection 4.1 is performed using DOS' to obtain r-Ol or o-Ol in terms of subregions or hypervolumes. The definition of DOS as either a nominal point or a region of setpoints differentiates $\mathrm{r}-\mathrm{Ol}$ and $\mathrm{o}-\mathrm{O}$.

An application to the classic shower problem illustrates this methodology. The same operability sets from Subsection 2.6 are considered. The DOS is defined as DOS $=$ $y^{N}=(5,84)$ for the regulatory case, whereas it is defined as DOS $=\left\{y \in \mathbb{R}^{2} \mid 3 \leq y_{1} \leq\right.$ 7 and $\left.74 \leq y_{2} \leq 94\right\}$ for the overall case. The r-Ol and o-Ol are then calculated using the above methodology with subregions. Figures 15 and 16 show the multimodel representation and calculation of $\mathrm{r}-\mathrm{Ol}$ and $\mathrm{o}-\mathrm{OI}$ respectively.
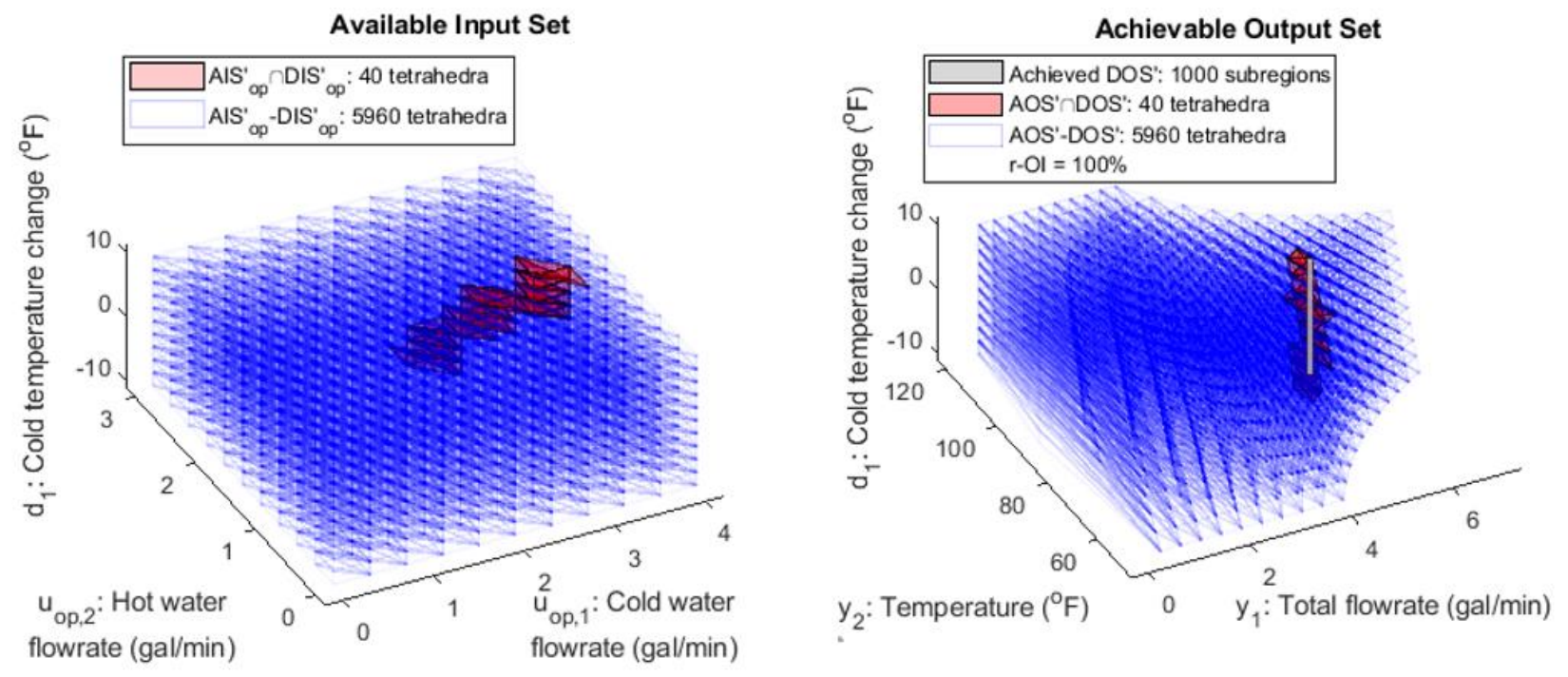

Figure 15. Multimodel calculations of $\mathrm{r}$-OI for the shower problem. 

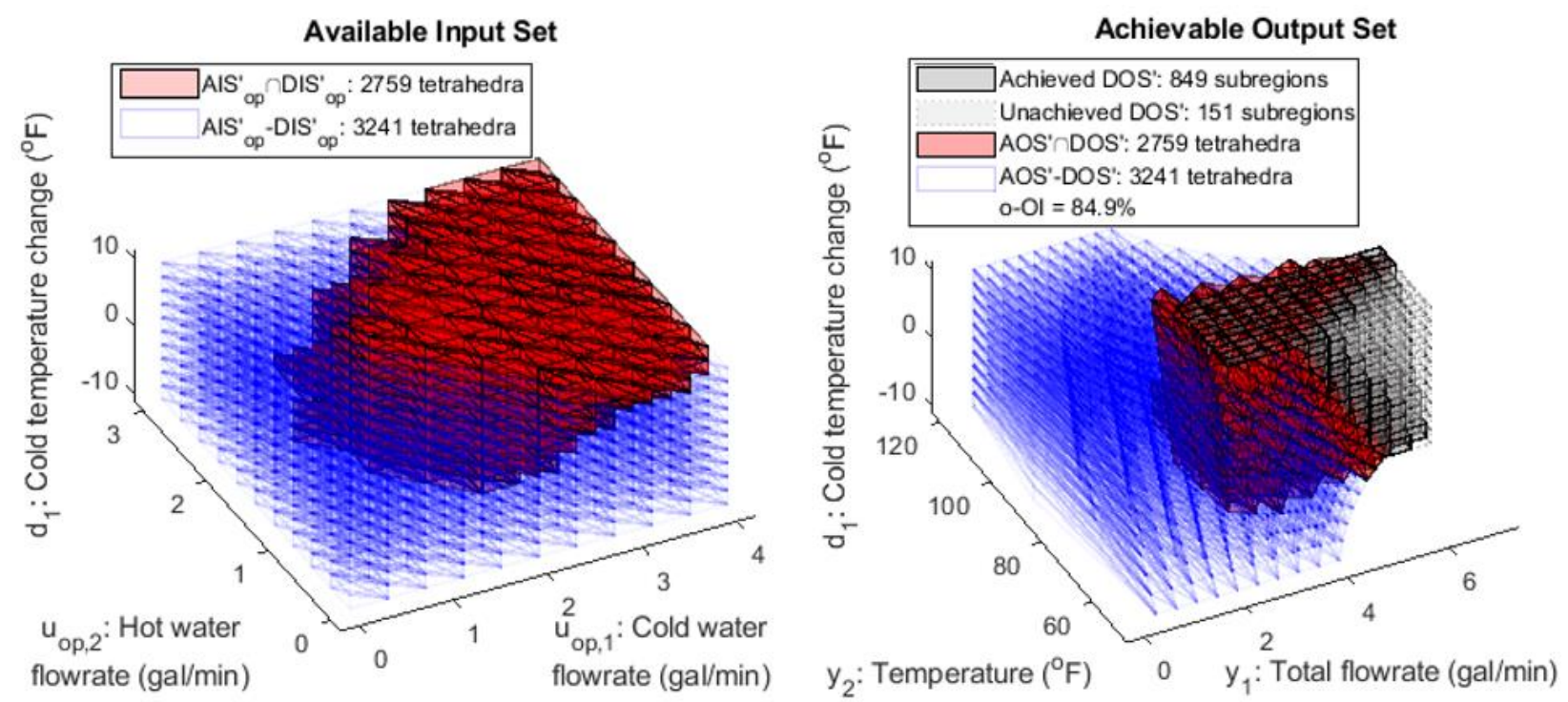

Figure 16. Multimodel calculations of o-OI for the shower problem.

These results indicate $\mathrm{r}-\mathrm{Ol}$ and $\mathrm{o}-\mathrm{OI}$ of $100 \%$ and $84.9 \%$, respectively. In Figure 15, DOS' corresponds to a line defined by $\operatorname{DOS}^{\prime}=$ $\left\{(y, d) \in R^{3} \mid y=y^{N}=(5,84)\right.$ and $\left.d_{1} \in[-10,10]\right\}$, which is completely inside AOS', indicating that $y^{N}$ is achievable in all EDS scenarios, i.e., all disturbances can be rejected. In Figure 16, the DOS' corresponds to a region defined by $\operatorname{DOS}^{\prime}=\left\{(y, d) \in \mathbb{R}^{3} \mid y \in\right.$ DOS and $\left.d_{1} \in[-10,10]\right\}$. The DOS' is divided into $10^{3}$ subregions from which 849 are achievable in various EDS scenarios.

Each EDS case can be analyzed as an $A O S^{\prime} \cap D O S^{\prime}$ cut in the $\mathrm{d}_{1}$ dimension. Figure 17 shows 3 cuts at $d_{1} \in\{-10,0,+10\}$. The disturbance $d_{1}=-10^{\circ} \mathrm{F}$ decreases the coldwater temperature, resulting in a wider range of final temperatures that the system can achieve, which corresponds to a larger $\operatorname{AOS}\left(d_{1}\right)$ across the $y_{2}$ axis in Figure 17a. The disturbance $d_{1}=+10^{\circ} \mathrm{F}$ limits the range of temperatures that the system can achieve because it brings the cold-water temperature closer to the hot-water temperature, producing a smaller $\operatorname{AOS}\left(d_{1}\right)$ across the $y_{2}$ axis in Figure 17c. The disturbance $d_{1}=0{ }^{\circ} \mathrm{F}$ is at the nominal disturbance value. Therefore, the cut in Figure 17b corresponds to the the servo case. Figure 17b presents similarities to Figures $13 \mathrm{a}$ and $13 \mathrm{~b}$ in Subsection 4.1 , where the shower problem is used to illustrate the calculation of s-OI in terms of subregions and hypervolumes. 
Here, for both $\mathrm{r}-\mathrm{OI}$ and o-OI, AOS' is not a result of the operation $\bigcap_{d_{1} \in E D S} \operatorname{AOS}\left(d_{1}\right)$, but a representation of $A O S \times E D S$. Therefore, achievability of the DOS is quantified for every disturbance scenario instead of being evaluated using the input perspective or the AOS region that is always achievable with respect to disturbances presented in Subsection 2.2.

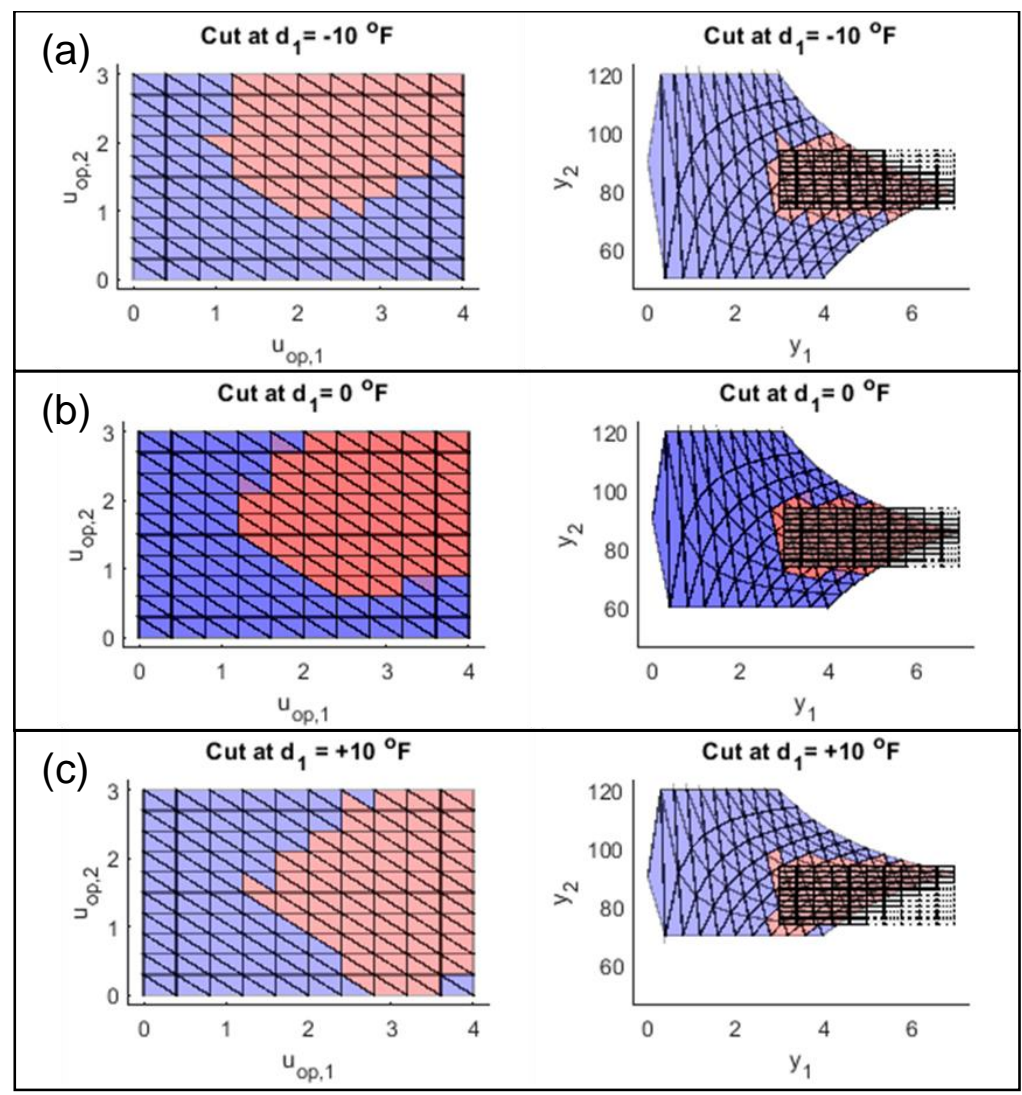

Figure 17. Cuts of the AISop'-AOS' mapping for the shower problem.

Note that the developed multimodel calculations of $\mathrm{Ol}$ in terms of subregions and hypervolumes rely only on the direct model, relaxing the need for $M^{-1}$ evaluations. An additional advantage is that the AOS is not modified using intersection operations, which eliminates details about behavior at each EDS scenario. The main limitations consist of restricting the system operation to the chosen AIS op and increasing the dimensionality of AIS'-AOS' mappings. In Subsection 7.3.4, this method is used for calculations of r-Ol and flexibility of a modular hydrogen unit. 


\section{Chapter 5 Multilayer Framework}

The developed multilayer operability framework tackles both the modular design and operational problems. The framework is developed with the assumption that the nonlinear system in focus is operating away from input-output singularities. In each layer, the original nonlinear model is substituted by a multimodel representation according to the adopted subsystem and definition of operability spaces as described above. The multilayer framework is explained in the subsection below, followed by a DMA-MR application.

\subsection{Multilayer Framework Description}

The proposed multilayer framework is entirely based on the multimodel representation. Candidate designs for SM are systematically selected and ranked according to operational performance. In the first layer, the iterative MILP-based algorithm is employed to find an MDR containing design candidates for SM and PI (modular design problem). In the second layer, the modular designs inside this region are systematically ranked using steady-state operability and the OI (operations problem). Figure 18 below summarizes the multilayer framework, which is comprised of 2 layers.

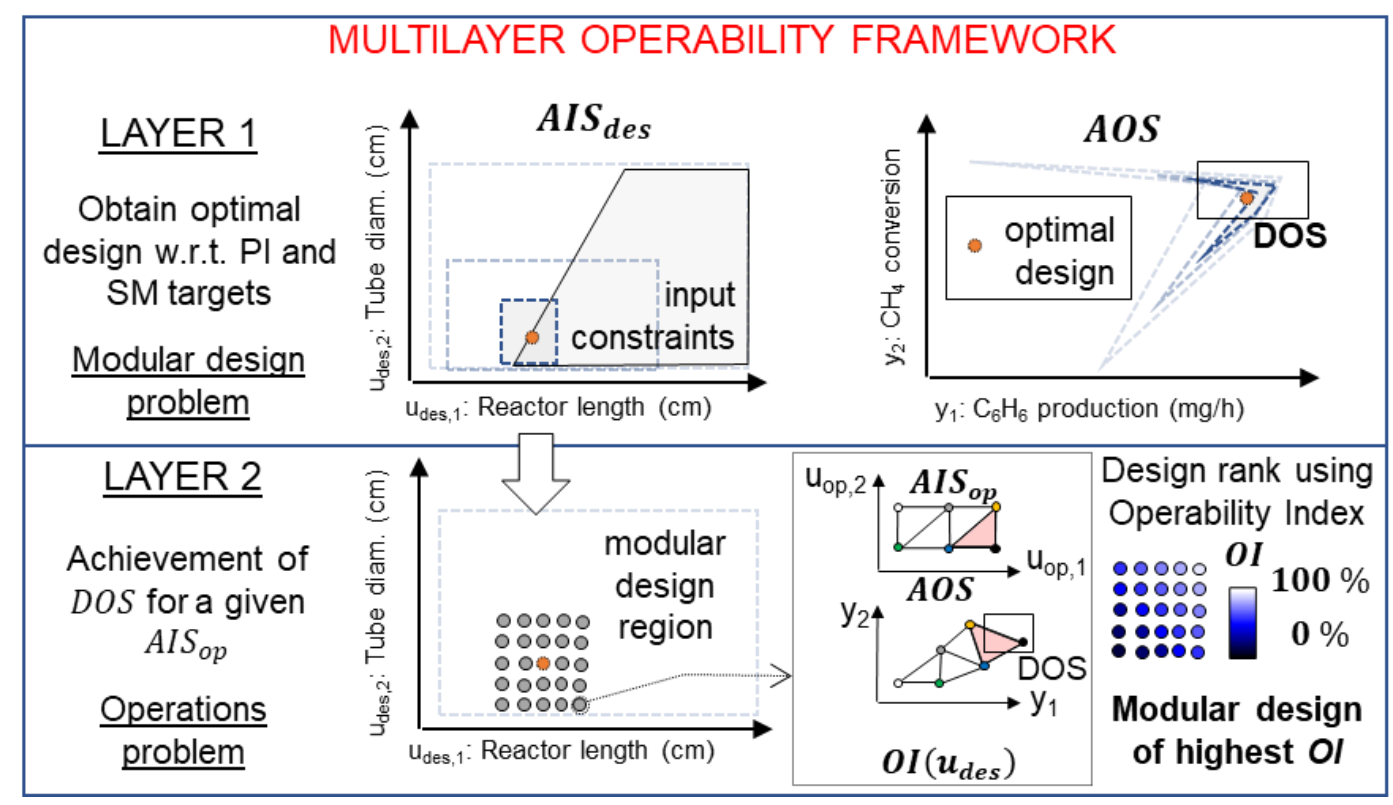

Figure 18. Multilayer framework scheme. 
At first, process operability sets are determined. The multilayer operability framework requires systems to have both design and operational inputs, and the presence of disturbance variables is optional. In the first layer, the MVs and, if present, disturbances, are fixed at a nominal operation for analysis of a AIS des $x$ AOS subsystem. A footprint objective function, PI targets and process constraints are included in the iterative MILP-based algorithm.

The iterative MILP-based algorithm calculates an optimal design with respect to PI and SM targets, and the MDR is built around the optimal design considering feasible construction values. Alternatively, the polytope of solution, $P_{s o l}$, or its bounds, $u_{i, P_{s o l}}^{\min }$ and $u_{i, P_{s o l}}^{\max }$, which are described in Subsection 3.2.2, can be selected as the MDR. This alternative is indicated for higher-dimensional systems in which convergence of the algorithm is likely to be computationally expensive. When adopted, criteria such as the number of iterations or the size of MDR should be employed.

In the second layer, the MDR is discretized, and the AIS op $\times$ AOS subsystem is analyzed for each considered design. For these analyses, the AIS op is strictly composed of MVs that would be used for control. Each design corresponds to one operational mapping described by the multimodel representation. If disturbances are present, the representation from Subsection 3.1.2 with AISop' x AOS' can be adopted.

One value of $\mathrm{Ol}$ is calculated for each $u_{d e s}^{M D R} \in M D R$. The Ol can be in terms of either subregions or hypervolumes and is obtained by employing the methods from Chapter 4. After the $\mathrm{Ol}$ is estimated for every design, the MDR is ranked according to operational performance. The OI quantifies the achievability of system objectives and capability of the design to reject disturbances. Higher values of Ols indicate designs with wider feasible operating regions and greater flexibility when disturbances are included. The multilayer framework is applied to a DMA-MR subsystem with 3 design inputs, 3 MVs and $3 \mathrm{CVs}$ below. The second layer of this framework is also applied in Subsection 7.3.4 to rank designs according to flexibility. 


\subsection{DMA-MR Application}

The multilayer framework is applied to a DMA-MR subsystem. The first layer finds the MDR, which is constructed from an optimal design point provided by the iterative MILPbased algorithm. The second layer classifies the MDR designs according to achievability of a DOS using the s-Ol. A comparison between the measures of $\mathrm{Ol}$ in terms of subregions and hypervolumes is established. Improvement of process operations and tradeoffs with footprint minimization are also analyzed.

The DMA-MR system from Subsection 3.2.3 is augmented by adding the input variable of membrane selectivity to the $A I S_{d e s}$, and the output variable of hydrogen production to the AOS. It is assumed here that the membrane selectivity could be improved in the lab if needed to achieve the desired process specifications. MVs are also included for classification of MDR, which configures an $A I S \in \mathbb{R}^{6}$ along with design variables. The sets $A I S_{\text {des }} \in \mathbb{R}^{3}$ and $A I S_{o p} \in \mathbb{R}^{3}$ are employed to distinguish the two types of inputs. The input variables are selected from Tables 2 and 3 and structured as follows:

$$
\begin{gathered}
u_{\text {des }, 1} \stackrel{\text { def }}{=} \text { Reactor length }(\mathrm{cm}) \\
u_{d e s, 2} \stackrel{\text { def }}{=} \text { Tube diameter }(\mathrm{cm}) \\
u_{d e s, 3} \stackrel{\text { def }}{=} \text { Selectivity }(-) \\
u_{o p, 1} \stackrel{\text { def }}{=} \text { Methane feed }\left(\mathrm{cm}^{3} / \mathrm{min}\right) \\
u_{o p, 2} \stackrel{\text { def }}{=} \text { Temperature }\left({ }^{\circ} \mathrm{C}\right) \\
u_{o p, 3} \stackrel{\text { def }}{=} \text { Sweep gas feed }\left(\mathrm{cm}^{3} / \mathrm{min}\right)
\end{gathered}
$$

where $u_{d e s}=\left(u_{d e s, 1}, u_{d e s, 2}, u_{d e s, 3}\right)$; and similarly, $u_{o p}=\left(u_{o p, 1}, u_{o p, 2}, u_{o p, 3}\right)$. For example, $(15,0.5,300) \in A I S_{\text {des }}$ is a design element associated with reactor length of $15 \mathrm{~cm}$, tube diameter of $0.5 \mathrm{~cm}$ and selectivity of 300 .

Using the variables and notation above along with the ranges from Tables 2 and 3 , the $A I S_{d e s}, A I S_{o p}$ and the complete AIS are sets, given, by

$$
\begin{aligned}
& A I S_{\text {des }}=\left\{u_{\text {des }} \in \mathbb{R}^{3} \mid(10,0.5,300) \leq u_{\text {des }} \leq\left(100,2.0,1 \times 10^{5}\right)\right\} \\
& A I S_{o p}=\left\{u_{o p} \in \mathbb{R}^{3} \mid(7,800,9) \leq u_{o p} \leq(9,1000,11)\right\} \\
& A I S=\left\{u \in \mathbb{R}^{6} \mid\left(u_{\text {des }}^{\min }, u_{o p}^{\min }\right) \leq u \leq\left(u_{\text {des }}^{\max }, u_{o p}^{\max }\right)\right\}
\end{aligned}
$$


where

$$
\left(u_{d e s}^{\min }, u_{o p}^{\max }\right)=(10,0.5,300,7,800,9) \quad \text { and }
$$

$\left(u_{d e s}^{\max }, u_{o p}^{\max }\right)=(100,2,1 \times$ $\left.10^{5}, 9,1000,11\right)$.

The AOS contains 3 outputs and is also a set in $\mathbb{R}^{3}$. The generation of the AOS can be obtained through direct mapping of the AIS elements using the process model $M$. Taking Table 4 as a reference and considering the AIS described by Equations (49) to (51), the following structure and definition of the AOS are obtained:

$$
\begin{gathered}
y_{1} \stackrel{\text { def }}{=} \text { Benzene production }(\mathrm{mg} / \mathrm{h}) \\
y_{2} \stackrel{\text { def }}{=} \text { Methaneconversion }(\%) \\
y_{3} \stackrel{\text { def }}{=} \text { Hydrogen production }(\mathrm{mg} / \mathrm{h}) \\
A O S=\left\{y \in \mathbb{R}^{3} \mid y=M(u) \text { and } u \in A I S\right\}
\end{gathered}
$$

where $y=\left(y_{1}, y_{2}, y_{3}\right)$.

Here, the multilayer framework focuses on the portions of the AOS that intersect the DOS. Considering the above structure of output variables and the desired ranges in Table 4, the DOS is given by

$$
D O S=\left\{y \in \mathbb{R}^{3} \mid(20,35,3) \leq y \leq(25,45,6)\right\}
$$

Given the dimensionality of the above defined sets, the utilization of the multilayer framework results in the sequential analysis of square systems. The following subsections contain the application of each layer of the framework and the comparison between classifications of MDR using subregions and hypervolumes.

\subsubsection{Modular Design Problem ( $1^{\text {st }}$ Layer)}

In the first layer of the framework, to obtain the MDR, the operational inputs are fixed at a nominal operation point $u_{o p}^{N} \in A I S_{o p}$, defined as $u_{o p}^{N}=(8,900,10)$. The AIS is thus limited in this layer, resulting in the subset $\left\{\left(u_{\text {des }}, u_{o p}^{N}\right) \in A I S \mid u_{d e s} \in A I S_{\text {des }}\right\}$, simplified to Equation (55) below.

$$
\text { AIS }_{\text {layer }_{1}}=A I S_{\text {des }} \times u_{o p}^{N}
$$

Note that $A I S_{\text {layer }_{1}}$ can be treated as a three-dimensional set, as only 3 coordinates associated with $A I S_{\text {des }}$ can be changed. As a result, a 3 × 3 square system containing 
design inputs and outputs associated with a fixed operation is analyzed in the first layer of this framework.

The same SM and PI targets from Subsection 3.2.3 are maintained, as well as the plug-flow constraint. The application of each of the steps of the iterative MILP-based algorithm is performed considering small changes from the previous case as described below. For illustration purposes, the presentation of the algorithm is simplified, containing only 3 iterations, the first, one intermediate $\left(4^{\text {th }}\right)$ and the last iteration $\left(8^{\text {th }}\right)$.

(1) Simulation: In the first iteration, with the addition of the membrane selectivity variable bounded as $u_{d e s, 3}=\left[3 \times 10^{2} \quad 1 \times 10^{5}\right], 3^{3}=27$ input-output points are obtained through simulation. In Figures 19 and 21, it is possible to see the formed grid for each iteration.

(2) Linearized Subsystems: Using the obtained grid, the Delaunay triangulation is performed and the set $S=\{1,2, \ldots, 48\}$ indicates the obtained set of polytopes, depicted in Figures 19 and 21. The six-dimensional joint multimodel representation is visually illustrated by two separate three-dimensional plots, resembling the disjoint representation.

(3) Quantification of Linear Spaces: The obtained multimodel representation in step 2 is used here. Similar to the two-dimensional case, a subset $S^{\prime} \subseteq S$ indicates the polytopes that satisfy both input and output constraints. The input and output regions can be found in Figures 20 and 22, respectively.

(4) Optimal Design: As the PI and SM targets and constraints are the same as in the previous case, the MILP formulation is changed solely by increasing its dimensionality from two to three dimensions as follows: 


$$
\begin{aligned}
& \chi \begin{array}{l}
\chi= \\
\qquad w_{j k}, b_{k}
\end{array} \\
& \text { Subject to } \\
& \quad 10 \leq u_{d e s, 1} \leq 100 \\
& 0.5 \leq u_{d e s, 2} \leq 2 \\
& 300 \leq u_{d e s, 3} \leq 1 \times 10^{5} \\
& 20 \leq y_{1} \leq 25 \\
& 35 \leq y_{2} \leq 45 \\
& 3 \leq y_{3} \leq 6 \\
& u_{d e s, 1} / u_{d e s, 2} \geq 30 \\
& \left.\left.b_{k} \in\{0,1\}, \quad k \in S_{d e s, 2}\right)\right) \\
& \sum_{k=1}^{K^{\prime}} b_{k}=1 \\
& \sum_{j=1}^{4} w_{j k}=b_{k}, k \in S^{\prime} \\
& 0 \leq w_{j k} \leq 1, \quad k \in S^{\prime}, \quad j \in\{1,2,3,4\} \\
& u_{i}=\sum_{k=1}^{K^{\prime}}\left(\sum_{j=1}^{4} u_{d e s, i, j k} \cdot w_{j k}\right), \quad i \in\{1,2,3\} \\
& y_{i}=\sum_{k=1}^{K^{\prime}}\left(\sum_{j=1}^{4} y_{i, j k} \cdot w_{j k}\right), \quad i \in\{1,2,3\}
\end{aligned}
$$

where $u_{d e s, 3}$ is the membrane selectivity, $y_{3}$ is the hydrogen production in $\mathrm{mg} / \mathrm{h}$ and the other variables follow the same notation adopted in the previous case.

Figures 20 and 22 show the selection of a tetrahedron in the $1^{\text {st }}, 4^{\text {th }}$ and $8^{\text {th }}$ iterations, as well as the calculation of the optimal points (plotted inside the figure).

(5) Stopping Criteria: As in the previous case, a tolerance error of $1 \%$ with respect to each variable is considered for convergence.

(6) New Bounds: Similar to the two-dimensional case, Figure 19 shows how the input bounds are reduced until convergence is obtained. 


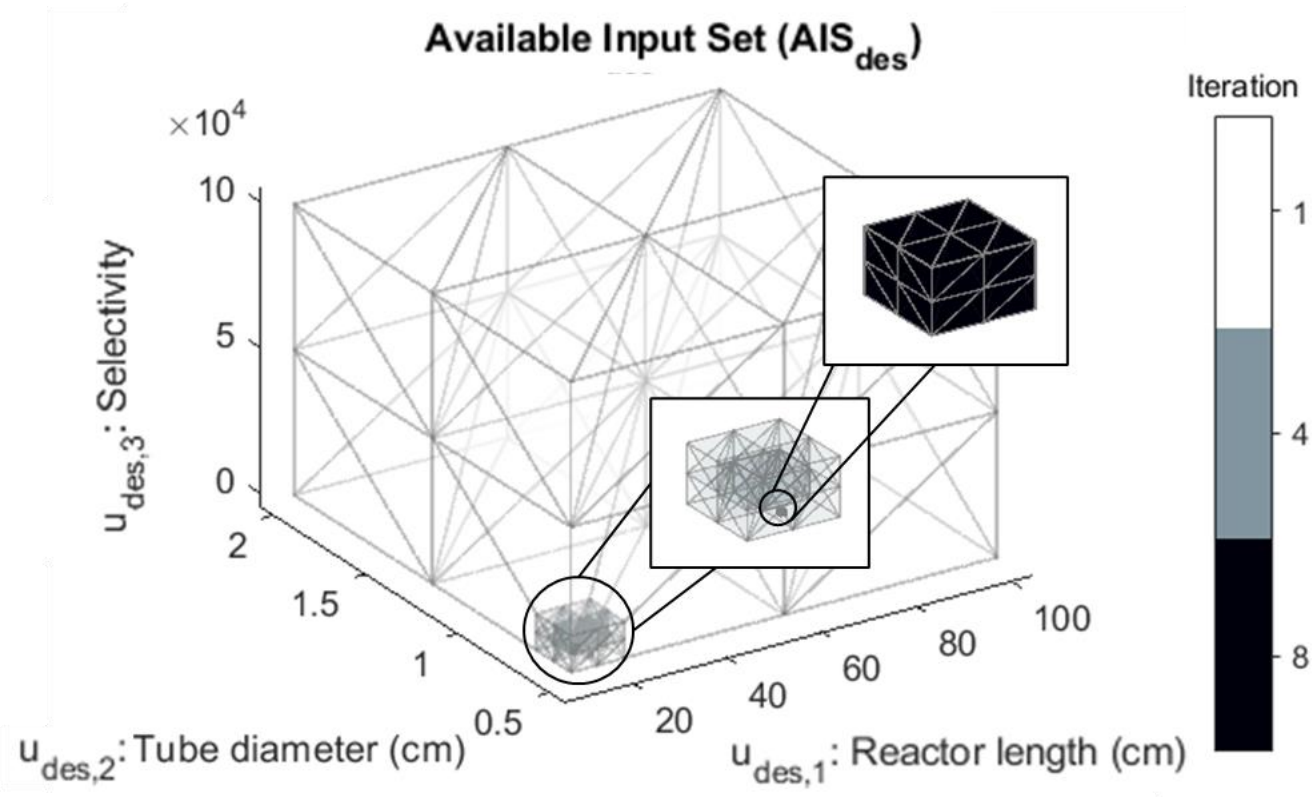

Figure 19. Three-dimensional: Input variable bounds and triangulations.

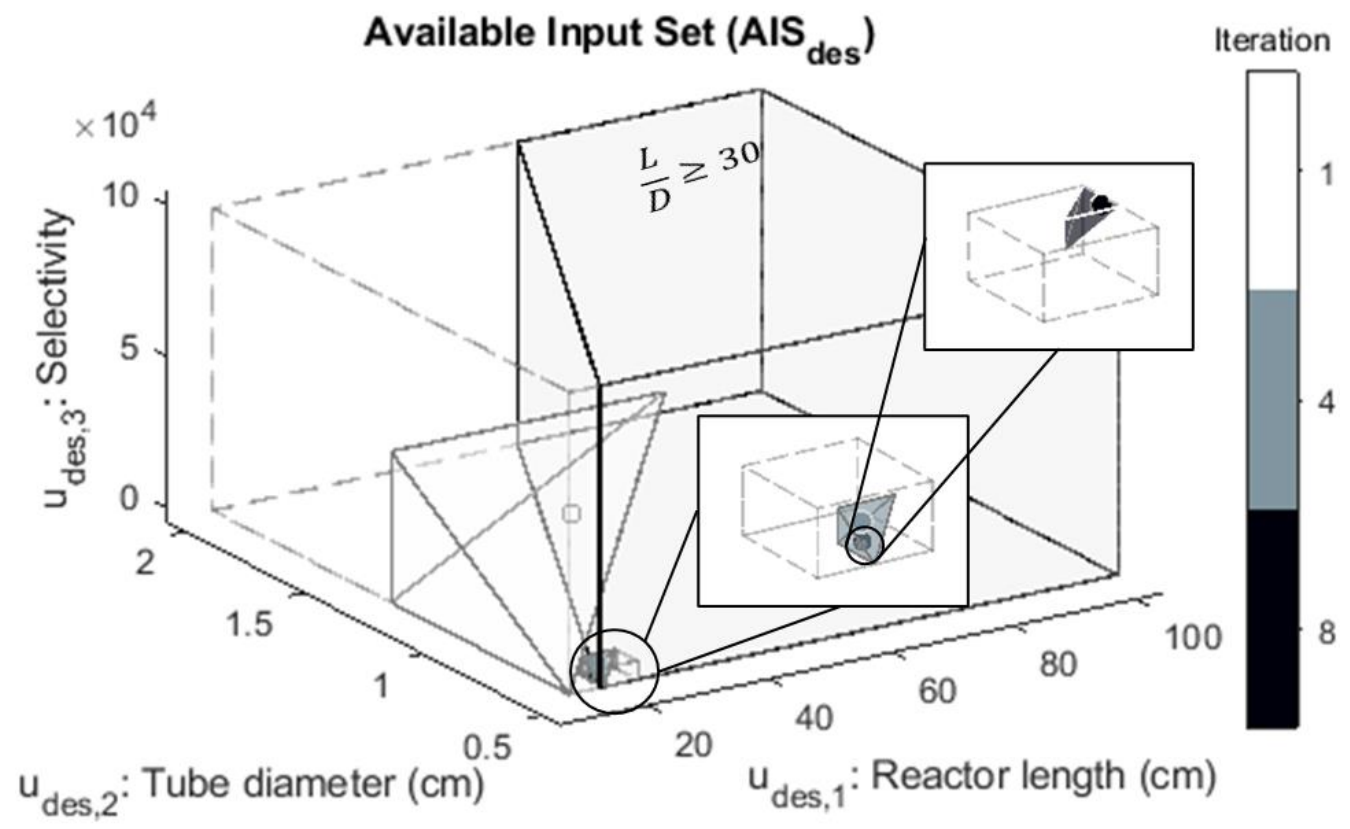

Figure 20. Three-dimensional: Selection of tetrahedra and optimal solution points. 

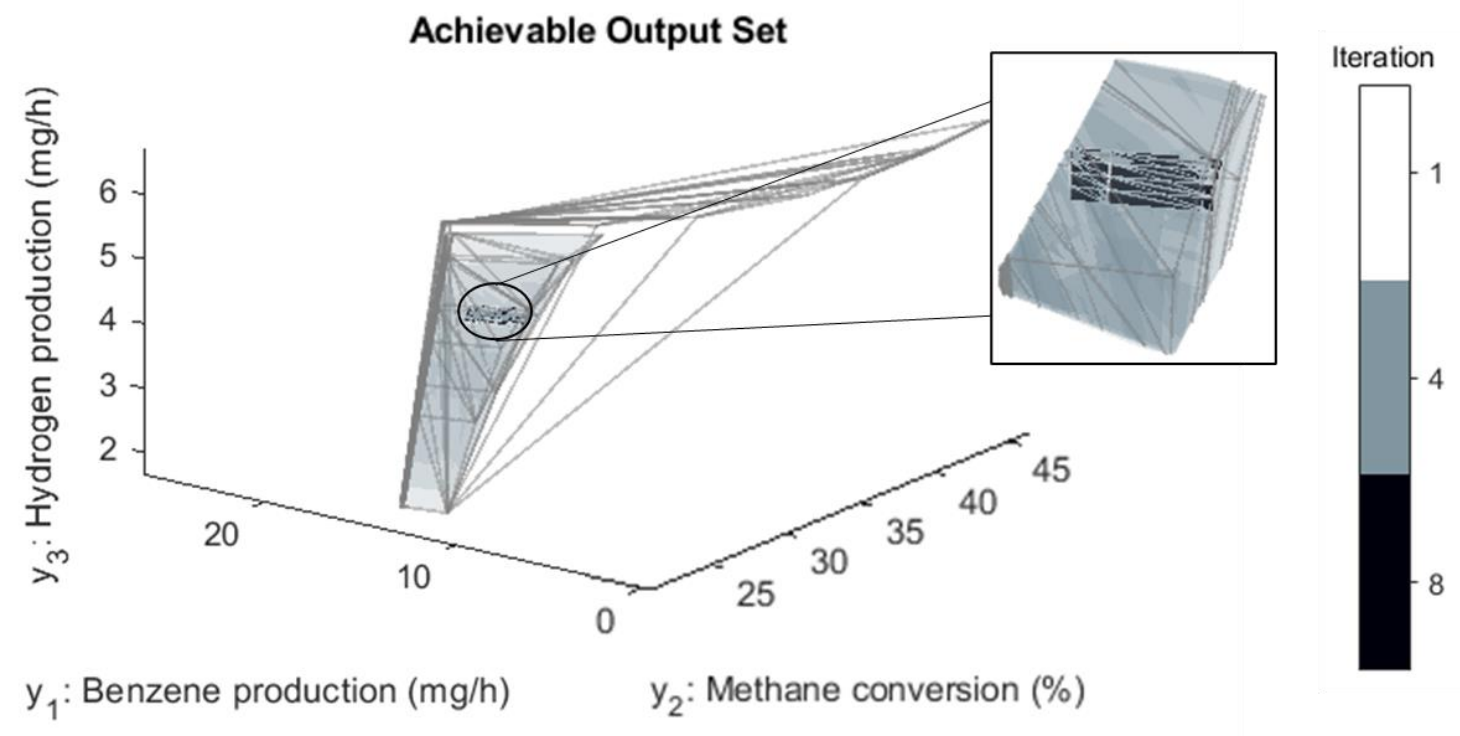

Figure 21. Three-dimensional case: Output mapping.
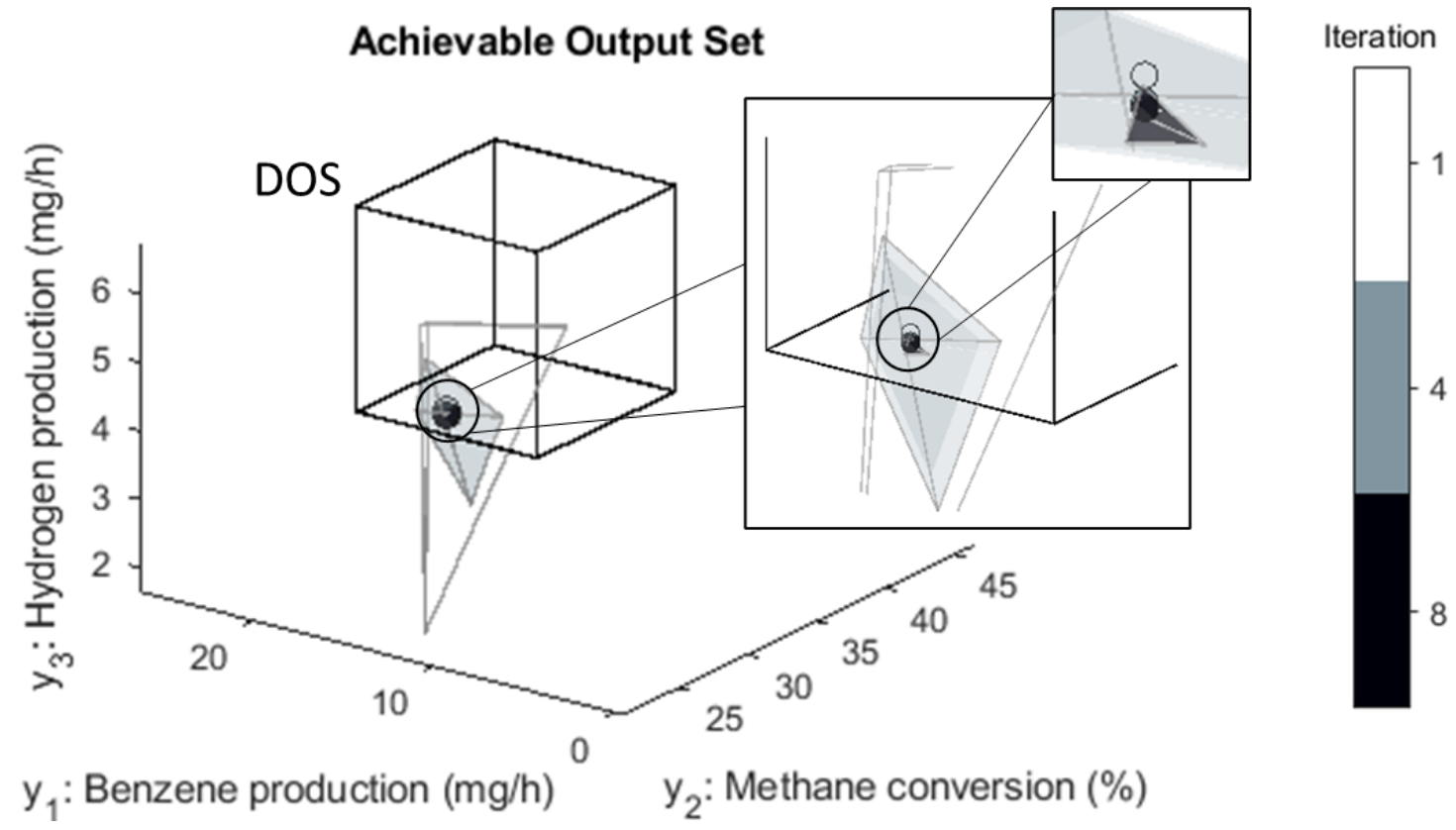

Figure 22. Three-dimensional: Selection of tetrahedra and optimal solution points.

The algorithm converges to a solution in 8 iterations and the result is a DMA-MR with reactor length of $16.99 \mathrm{~cm}$, tube diameter of $0.57 \mathrm{~cm}$ and membrane selectivity of 1037 that corresponds to a benzene production of $20 \mathrm{mg} / \mathrm{h}$, methane conversion of 0.35 
and hydrogen production of $3.29 \mathrm{mg} / \mathrm{h}$. The total computational time for the algorithm convergence is 34 seconds.

The MDR is built around the optimal point with values that would be more reasonable for construction: reactor lengths from 16 to $18 \mathrm{~cm}$, tube diameters from 0.5 to $0.6 \mathrm{~cm}$ and membrane selectivities from 500 to 1500 . The MDR is mathematically represented by

$$
M D R=\left\{u_{\text {des }} \in \mathbb{R}^{3} \mid(16,0.50,500) \leq u_{\text {des }} \leq(18,0.60,1500)\right\}
$$

\subsubsection{Operations Problem ( $2^{\text {nd }}$ Layer $)$}

First, the MDR is discretized. Steps of $0.2 \mathrm{~cm}, 0.01 \mathrm{~cm}$ and 100 are applied to reactor length, tube diameter and membrane selectivity, respectively, to obtain rounded values that would be suitable for manufacturing the possible modular designs. The result is a total of $11^{3}=1,331$ possible designs that cover approximately $\pm 5 \%$ around the obtained optimal design, representing a MDR constructed based on the outcome of the first layer of the framework.

For each of the 1,331 possible design points in the AIS des, the design is fixed, and an AISop-AOS operability analysis is performed keeping the same DOS listed in the $3 \times 3$ case above. Therefore, for each element $u_{d e s}^{M D R} \in M D R$, the subset of the AIS $\left\{\left(u_{\text {des }}^{M D R}, u_{o p}\right) \in A I S \mid u_{o p} \in A I S_{o p}\right\}$, is simplified to

$$
A I S_{\text {layer }}=u_{\text {des }}^{M D R} \times A I S_{o p}
$$

For each AISop-AOS mapping, a disjoint multimodel representation is obtained from 27 input-output simulated points. Similarly, the obtained mapping is given by a set of 48 paired tetrahedra, i.e., $\left\{P_{k} \mid k \in S\right\}$ and $S=\{1,2, \ldots, 48\}$. This multimodel representation can be seen in Figures 23 and 24.

To quantify the achievability of each design by the s-OI, the DOS is evenly divided into 125 subregions, represented by 125 parallelepipeds. The number $5^{3}=125$ is chosen to define parallelepipeds in which the sides have lengths of $1 \mathrm{mg} / \mathrm{h}$ for benzene 
production, 0.02 for methane conversion and $0.6 \mathrm{mg} / \mathrm{h}$ for hydrogen production. The s-OI corresponds to the percentage of these 125 subregions that a considered design can achieve, given the described AIS op Figures 23 and 24 contain examples of the divided DOS and calculations of the s-OI. In Figure 23, 22 of 125 subregions of the DOS are achieved, corresponding to an s-OI value of $17.6 \%$. Whereas, in Figure 24, only 2 of these subregions are achieved, corresponding to an s-Ol value of $1.6 \%$.

After the described operability analysis is completed for all points of the MDR, the values of s-Ol are used to rank the considered designs. From this ranking, the design of maximum s-Ol is then selected, consisting of a design with reactor length of $18 \mathrm{~cm}$, tube diameter of $0.6 \mathrm{~cm}$ and membrane selectivity of 1,500. Figure 25 shows the rankings using values of s-OI, the selected design of maximum s-OI and the previously obtained optimal design, rounded to reactor length of $17 \mathrm{~cm}$, tube diameter of $0.57 \mathrm{~cm}$ and membrane selectivity of 1,000 .

Figures 23 and 24 represent the individual AISop-AOS operability analysis of the design of maximum s-OI (17.6\%) and the previously obtained optimal design (with s-OI of $1.6 \%$ ), respectively. For both cases, the model inversion $M^{-1}[D O S \cap A O S]$ is performed using connected tetrahedra to produce additional comparative information. The resulting paired polytopes from this operation are identified in red.
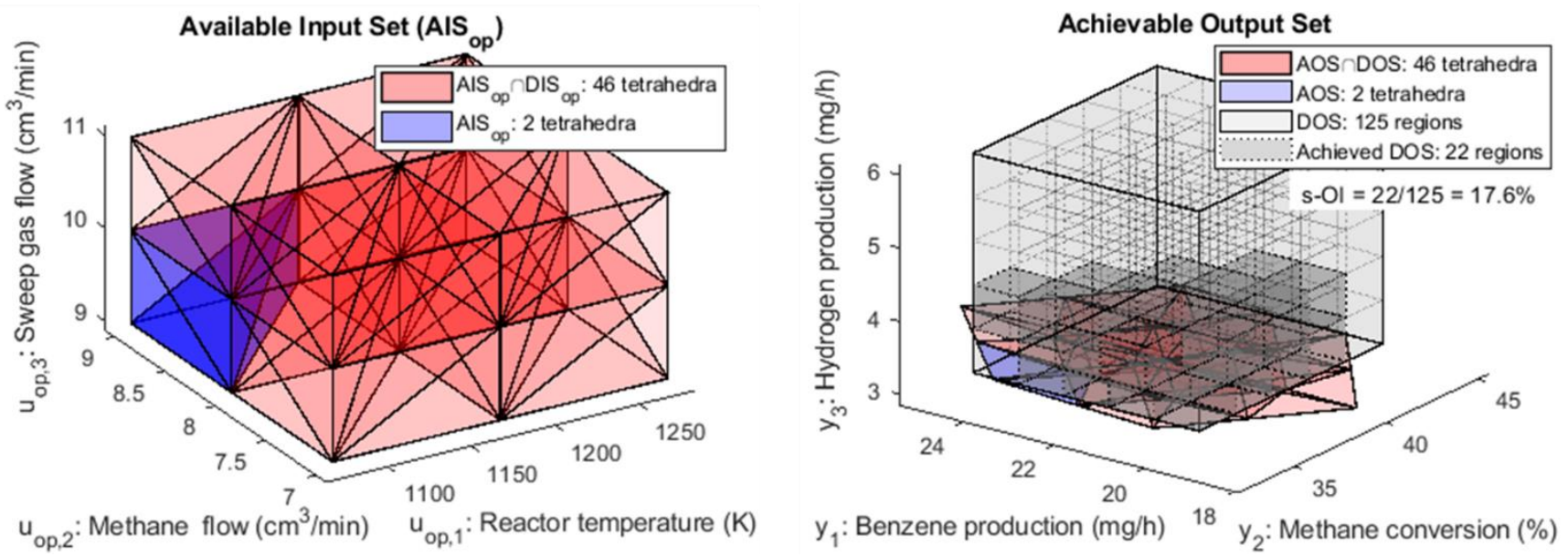

Figure 23. s-Ol and model inversion for design of highest s-Ol. 

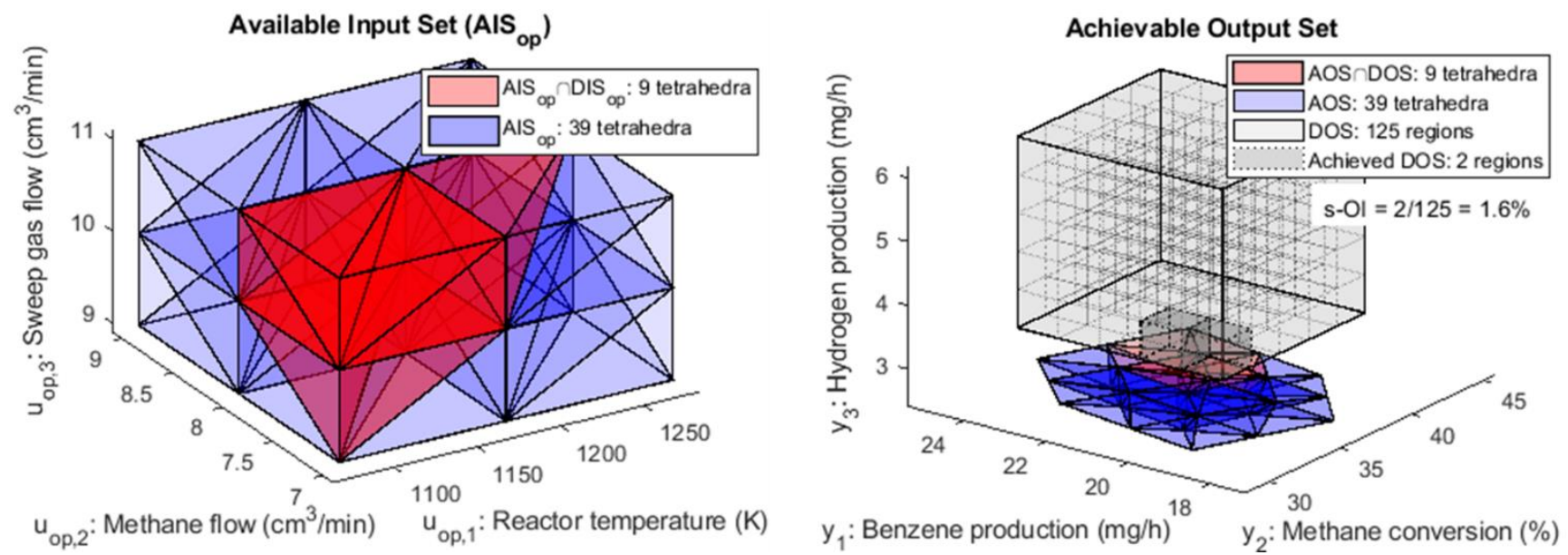

Figure 24. s-Ol and model inversion for previously obtained optimal design.

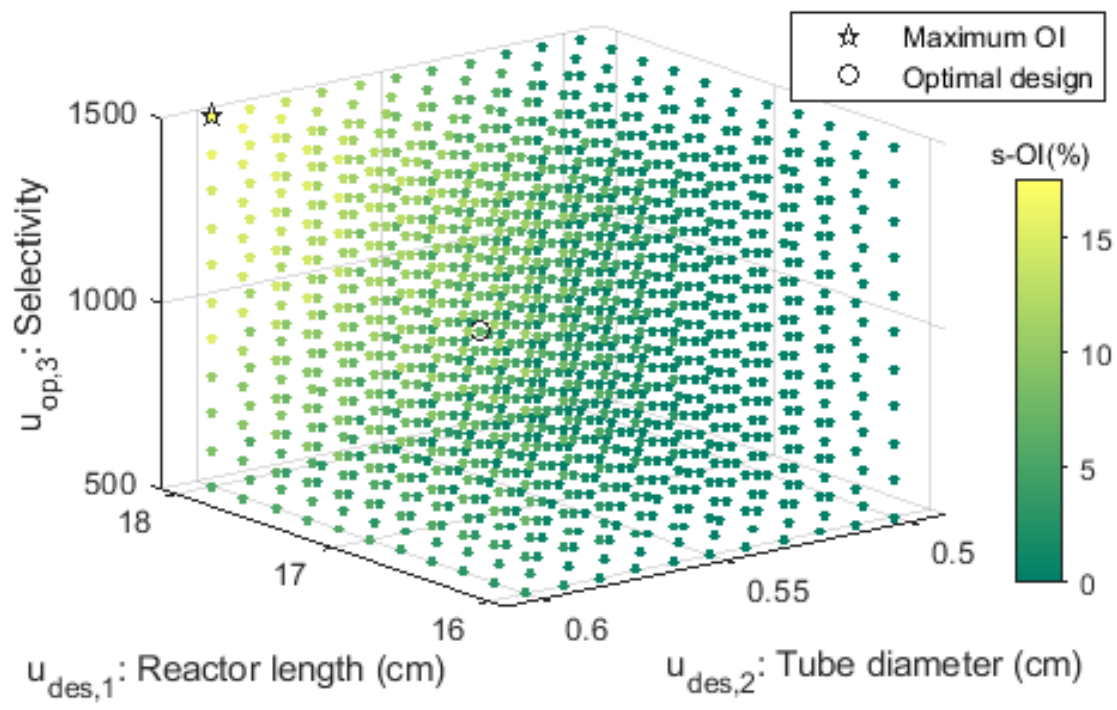

Figure 25. Achievability analysis of MDR designs using s-OI.

When contrasted with the design of maximum s-OI, the optimal design presents lower values for reactor length, tube diameter and membrane selectivity. However, the number of DOS achieved subregions is 2 , which consists of a relatively low achievability when compared to the best s-OI case, with 22 achieved subregions. Also, from the 48 tetrahedra that represent the available operational inputs of the AISop, the highest s-OI design covers 46 tetrahedra while the optimal design covers only 9, indicating a better exploitation of the AIS op for the achievement of the DOS in the highest s-OI case. 
The presented result can be attributed to the fact that the iterative algorithm computes an optimal point of AIS des for a fixed operating condition (a point of the AISop). The goal of the algorithm is solely to minimize the objective function, given by the linearized footprint, without considering process operation. The operability analysis presented above indicates that slightly bigger reactors are more operable with respect to the considered AIS op and DOS. Moreover, the trends in Figure 25 indicate that because higher values of $\mathrm{s}-\mathrm{Ol}$ are obtained for larger reactors, the objectives of minimizing size and maximizing s-Ol are conflicting in this case. Future work should consider other objectives such as a cost measure associated with membrane selectivity.

Taking the design of highest s-Ol as the most operable design, an estimation of a multitubular reactor is made. Assuming a shell of diameter of $50 \mathrm{~cm}$, length of $18 \mathrm{~cm}$ and approximate distance of $0.7 \mathrm{~cm}$ among adjacent tubes, a total amount of 1,111 tubes can be placed inside the shell. The obtained multitubular modular design can convert $113 \mathrm{ft}^{3}$ of methane to $574 \mathrm{~g}$ of benzene and $94 \mathrm{~g}$ of hydrogen per day.

A commercial truck as specified in Table 1 could transport the approximate maximum of 2,225 modules. In a hypothetical scenario in which all these modules are installed, a total consumption of about $252 \mathrm{Mcf} /$ day of methane would take place. A well in the Marcellus Shale Formation can produce from $500 \mathrm{Mcf} /$ day to $12 \mathrm{MMcf} /$ day of NG depending on the well maturity ${ }^{63,64}$. Thus, from 2 up to 24 trucks, if used to transport the modular units, could allow the onsite utilization of NG from a typical well in this region.

\subsubsection{MDR Ranking using Subregions and Hypervolumes}

The MDR rank using s-OI is compared for the measures in terms of subregions and volume. For each measure, the complete rank using $s-O I\left(u_{d e s}^{M D R}\right)$ is obtained for each element $u_{d e s}^{M D R} \in M D R$. Figures 26 and 27 below depict the classification of the modular region $M D R$ using $\mathrm{s}-\mathrm{O}$ in terms of subregions and volume, respectively. 


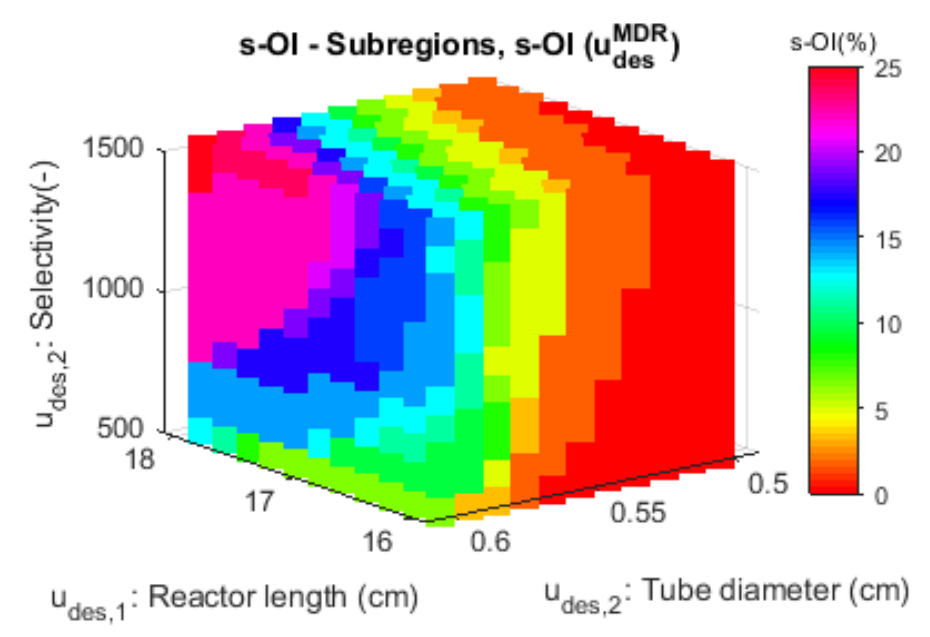

Figure 26. Classification of MDR using s-OI in terms of subregions.

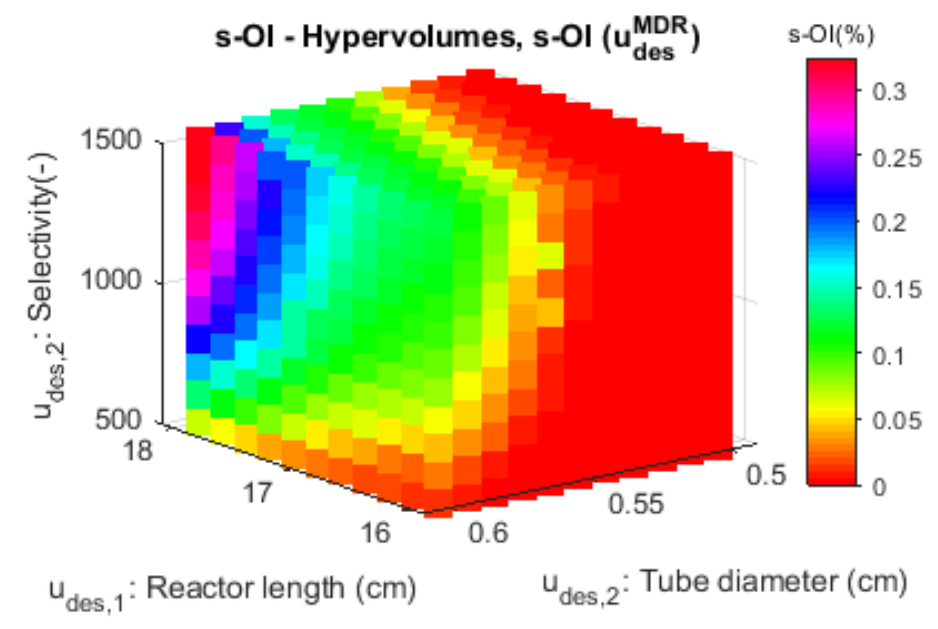

Figure 27. Classification of MDR using s-OI in terms of hypervolumes.

The s-OI measures using subregions are clearly higher, ranging from 0 to $25 \%$. The measures using volume range from 0 to approximately $0.3 \%$. This difference in magnitude happens because the subregions only require the presence of one point of the AOS to be considered achieved. Therefore, the proportion of achieved subregions tend to be higher than the proportion of achieved volume.

Analyzing the distribution of values of s-OI inside MDR, Figures 26 and 27 show that both measures present similar trends. Larger membrane reactors provide higher s- 
Ols and thus more achievability of the DOS. This classification using s-OI is in accordance with the previously applied $2^{\text {nd }}$ layer of the framework in Subsection 5.2.2.

The differences in the magnitude of s-OI do not significantly affect the selection of the design with maximum achievability for this application. However, they show that the interpretation of s-Ol in terms of volume may be misleading in some cases. The shape of the AOS is key to understand why this measure can have such low values. 


\section{Chapter 6 Process Operability App}

The software infrastructure contribution in this dissertation is an open-source platform named "Process Operability App Project". Algorithms described in this dissertation are structured and compiled in the form of a MATLAB app. A significant effort was dedicated to make the involved scripts as generic as possible by both addressing a variety of system dimensionalities and writing codes as functions of user-defined process models, sets and configurations.

In addition to the inclusion of the algorithms, a user-interface is developed to allow a versatile user-friendly utilization of the developed tools. The NLP-based and the multimodel approaches are accessible through functionalities such as: (i) generate inputoutput points; (ii) obtain the multimodel representation; (iii) calculate OI; (iv) find a feasible DIS; and (v) obtain an optimal design or a design region. All the computational geometry calculations in the app are performed using MPT. ${ }^{58}$ In terms of dimensionality limitations, preliminary tests indicate that the NLP-based approach is essentially restricted by computational time and the multimodel approach currently handles calculations involving polytopes with up to 5 dimensions. New releases will pursue the increase in system dimensionality and optimization of computational time with the objective of tackling problems of increased complexity. More information can be found in the app documentation provided in the website mentioned below.

This initiative aims to not only aid process systems applications with the use of operability approaches, but also to promote dissemination and discussion in academia and industry towards the improvement of the process operability field. The download of the Process Operability App and additional information are available at:

\section{https://fernandolima.faculty.wvu.edu/operability-app}

All the results presented in this dissertation were generated using the Process Operability App. Other applications are selected in this chapter to demonstrate the capability of app using the NLP-based and additional tools. The DMA-MR and the cycling of a carbon capture system (CCS) of a coal-fired power plant are complex energy systems considered for this task. The DMA-MR is a subsystem of the application presented in 
Subsection 3.2.3, and the CCS is part of an effort to integrate coal-fired power plants with renewable energy. 65

\subsection{Carbon Capture System Application}

With the increasing penetration of renewable energy into the power grid, traditional coalbased technologies have to be gradually integrated with wind, solar, and other clean energy sources. The retrofit of existing plants is an important example of how these new forms of energy can be coordinated with reliable conventional technologies.

For this application, a CCS unit is analyzed for implementation in a coal-fired power plant. Particularly, to achieve the required power demand, the intermittent behavior of solar and wind energy can be integrated with the energy generation from coal. A consequent cycling profile is needed from the perspective of the coal-fired power plant, producing variable amounts of flue gas in a day. The CCS unit receives and treats this flue gas, capturing and thereby limiting the emissions of $\mathrm{CO}_{2}$.

A candidate design of the CCS unit is analyzed here, considering the ranges of MVs and expected amounts of flue gas. The goal of this operability analysis is to determine the maximum $\mathrm{CO}_{2}$ capture for the employed design and provide insights for possible improvements in operation and design. Here, no quantification of $\mathrm{Ol}$ is performed, but instead, the input-output mapping with a focus on finding the AOS is explored through the Operability App.

For the CCS system, the selected inputs are exclusively operational, i.e., AIS = $A I S_{o p}$. Besides the flue gas flowrate from the coal-fired power plant, two streams of the CCS are selected. They are associated with the carbon absorption and stripping by the aqueous monoethanolamine (MEA) solvent. The outputs are variables associated with carbon capture, including the amount of employed solvent and overall work of the CCS unit. Table 5 shows the input variables and selected ranges. Equations (59) and (60) below describe the inputs' structure and the AISop, respectively. Equations (61) and (62) present the outputs' structure and the corresponding AOS. 
Table 5. Input variables and available ranges for CCS application.

$$
\begin{aligned}
& \text { operational input variable available range } \\
& \text { Flue gas flowrate }(\mathrm{kmol} / \mathrm{s}) \quad 3.34-3.70 \\
& \text { Lean MEA solvent flowrate (kmol/s) } \quad 9.51-10.75 \\
& \text { Low pressure steam flowrate }(\mathrm{kmol} / \mathrm{s}) \quad 1.33-1.47 \\
& u_{o p, 1} \stackrel{\text { def }}{=} \text { Flue gas flowrate }(\mathrm{kmol} / \mathrm{s}) \\
& u_{o p, 2} \stackrel{\text { def }}{=} \text { Lean MEA solvent flowrate }(\mathrm{kmol} / \mathrm{s}) \\
& u_{o p, 3} \stackrel{\text { def }}{=} \text { Low pressure steam flowrate }(\mathrm{kmol} / \mathrm{s}) \\
& A I S_{o p}=\left\{u_{o p} \in \mathbb{R}^{3} \mid(3.34,9.51,1.33) \leq u_{o p} \leq(3.70,10.75,1.47)\right\} \\
& y_{1} \stackrel{\text { def }}{=} \mathrm{CO}_{2} \text { capture rate (\%) } \\
& y_{2} \stackrel{\text { def }}{=} \text { Lean solvent } \mathrm{CO}_{2} \text { loading }\left(\mathrm{mol}_{\mathrm{CO}_{2}} / \mathrm{mol}_{\mathrm{MEA}}\right) \\
& y_{3} \stackrel{\text { def }}{=} \text { CCSoverallwork }(\mathrm{kW}) \\
& A O S=\left\{y \in \mathbb{R}^{3} \mid y=M\left(u_{o p}\right) \text { and } u_{o p} \in A I S_{o p}\right\}
\end{aligned}
$$

where $M$ refers to the process model, which is the reduced-order model briefly described below.

The three input variables are assumed to be within the ranges that are also adopted to obtain the reduced-order models for the coal-fired power plant. The reduced model for the CCS unit is obtained from previously developed work by employing system identification techniques. Details about these techniques, the process flowsheet, and the cycling operation can be found in ref. ${ }^{66}$.

For this study, the flue gas flowrate variable is assumed to be a measured disturbance as it comes from upstream units of the coal-fired power plant, and the other two inputs are manipulated variables. Here, $u_{o p, 1}$ is not assigned to the EDS as it is assumed to be controlled by other portions of the plant. Moreover, this variable has a specific expected profile that has higher values in the periods of morning and night when solar energy generation is low and energy demand is high. ${ }^{66}$

Using the Operability App, the reduced-order model is uploaded, and the input variable ranges are discretized. The plant behavior is simulated for several combinations 
of inputs within the formed grid, generating the input-output mapping shown in Figure 28 below. In this figure, the color code indicates the correspondence between the mapped input and output points.
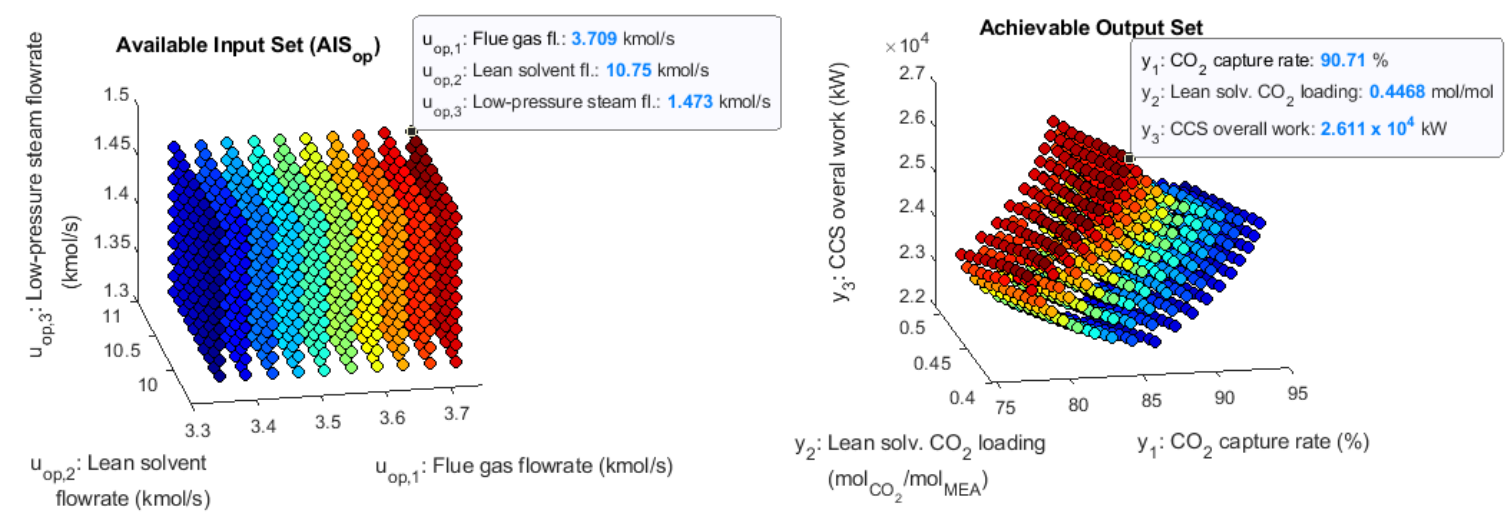

Figure 28. Input-output mapping of CCS unit.

For each value, uop, 1 , of the flue gas flowrate, the ranges of lean solvent flowrate and low-pressure steam flowrate can be combined to form a two-dimensional space of possibilities where these two manipulated variables can be set. In Figure 28, such spaces are represented in the AISop by "flat sheets" of blue, light blue, green, yellow, and so on.

The corresponding outputs are also sheet surfaces that are distributed in the AOS. For low values of the flue gas flowrate (dark blue points in Figure 28), the achievable space is a surface located in an AOS region of higher $\mathrm{CO}_{2}$ capture rate and smaller values for the lean solvent $\mathrm{CO}_{2}$ loading and CCS overall work. Conversely, high values of the flue gas flowrate (red points in Figure 28) generate an AOS surface in which the $\mathrm{CO}_{2}$ capture rate is lower and the lean solvent $\mathrm{CO}_{2}$ loading and CCS overall work are higher. This behavior indicates that the considered design is capable of achieving higher $\mathrm{CO}_{2}$ capture rates with smaller operating costs as expected when lower amounts of flue gas have to be processed; while higher amounts of flue gas limit the achievable $\mathrm{CO}_{2}$ capture rates and increase the operating cost of the unit associated with the lean solvent $\mathrm{CO}_{2}$ loading and CCS overall work.

To quantify the limited achievability for the $\mathrm{CO}_{2}$ capture rate, the worst-case scenario for the variable Uop, 1 (corresponding to the flue gas flowrate of around $3.7 \mathrm{kmol} / \mathrm{s}$ ) is analyzed. Figure 28 contains a highlighted point that corresponds to a possible 
maximum $\mathrm{CO}_{2}$ capture rate for this scenario. Note that when the CCS unit receives a flue gas flowrate at its highest expected value, the MVs of the lean solvent flowrate and lowpressure steam flowrate can be set to their corresponding upper limits to achieve a maximum $\mathrm{CO}_{2}$ capture of around $90.7 \%$.

To further verify the limitation on $\mathrm{CO}_{2}$ capture rates of the CCS design, a DOS is first set to a minimum value of $\mathrm{CO}_{2}$ capture rate of $90 \%$ and then slightly increased to a rate of $92 \%$. The input-output mapping is converted to the multimodel representation and, for each case, the achievability of the DOS is evaluated. The DISop is also calculated for each case, consisting of the input region that would be needed to achieve the analyzed DOS. For both DOSs, the Operability App is employed to perform the analysis and generate the plots.

Figure 29 shows the operability analysis for a $\mathrm{CO}_{2}$ capture rate equal to or higher than $90 \%$. In this figure, the red and blue regions refer to the portions that achieve and do not achieve the $\mathrm{CO}_{2}$ capture goal, respectively. The higher the flowrate of flue gas, the more restricted the available region is to achieve the desired $\mathrm{CO}_{2}$ capture rate of $90 \%$. Moreover, the needed values for the MVs of lean solvent flowrate and low-pressure steam flowrate are more limited and closer to their upper bounds as the flue gas flowrate increases.
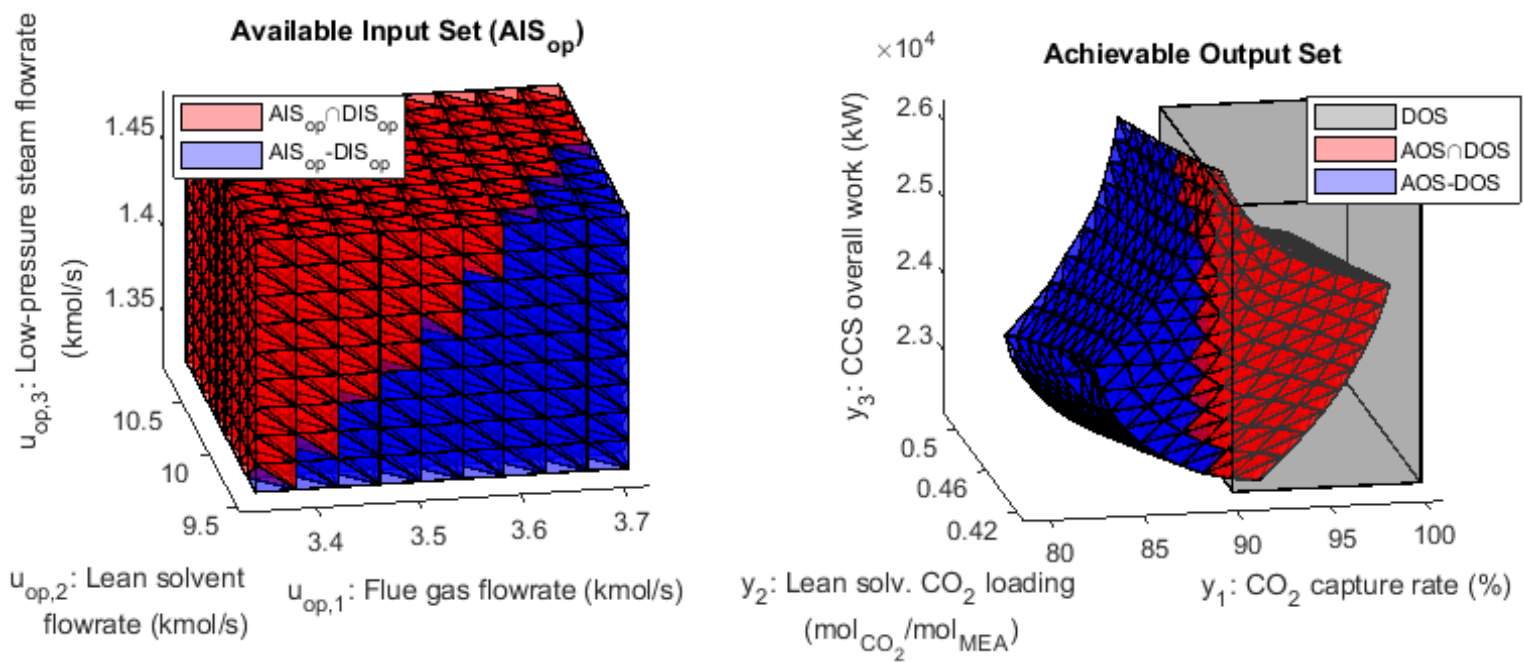

Figure 29. Steady-state achievability of $90 \%$ of $\mathrm{CO}_{2}$ capture rate. 
Figure 30 depicts a similar study for the desired $\mathrm{CO}_{2}$ capture rate of $92 \%$. By inspecting the AISop regions, it is possible to see that for values of flue gas flowrate in the range around $3.62-3.7 \mathrm{kmol} / \mathrm{s}$, there are no combinations of lean solvent flowrate and low-pressure steam flowrate that can take the system response to the DOS (all combinations are in blue, outside of the DIS $\mathrm{op}_{\mathrm{p}}$. Therefore, for this flue gas configuration, the CCS design is not capable of achieving the $\mathrm{CO}_{2}$ capture rate of $92 \%$.
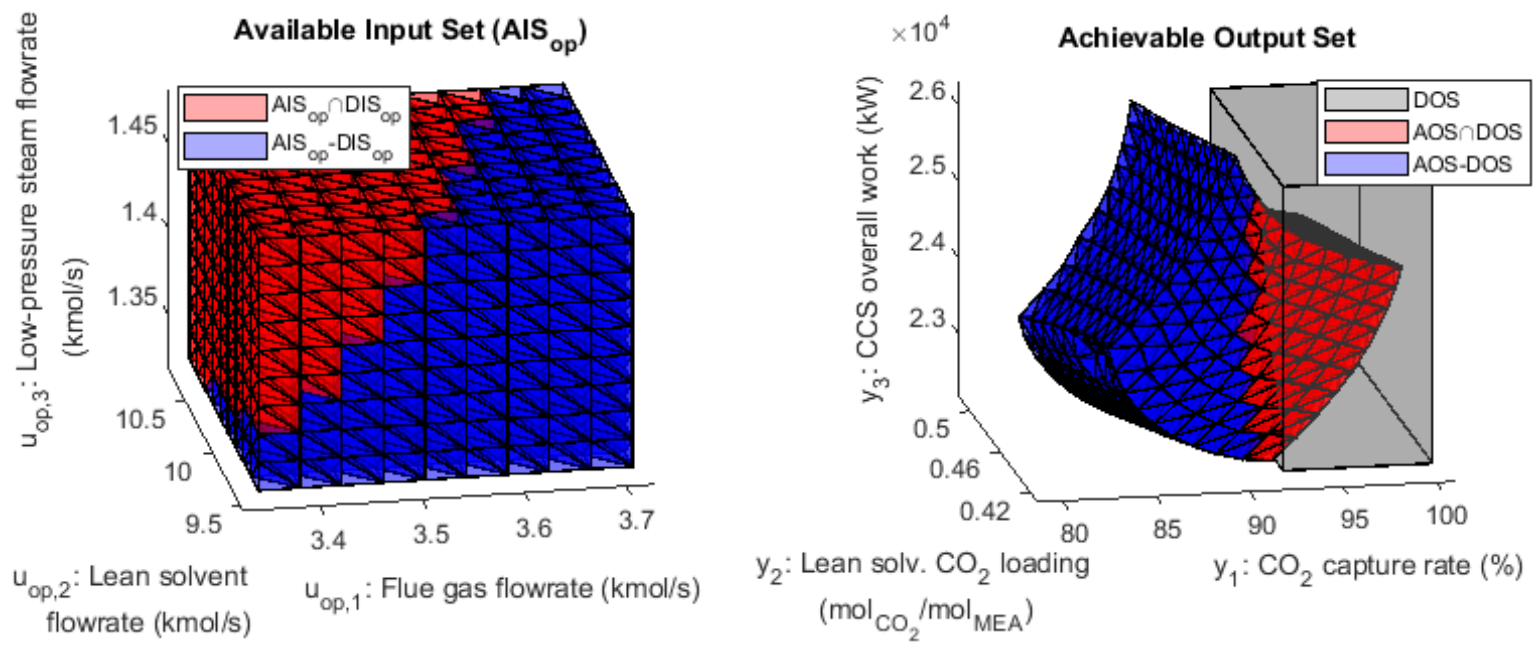

Figure 30 . Steady-state achievability of $92 \%$ of $\mathrm{CO}_{2}$ capture rate.

From the employed operability analysis, considering all flue gas flowrate scenarios, the current CCS design can achieve the standard $\mathrm{CO}_{2}$ capture rate of $90 \%$ with the available ranges of lean solvent and low-pressure steam flowrates. However, this CCS design is not able to achieve $\mathrm{CO}_{2}$ capture rates above $91 \%$ for all the flue gas flowrate scenarios. To enable higher $\mathrm{CO}_{2}$ capture rates, process design changes would have to be considered, such as enlarging the available ranges of MVs or changing the CCS design, e.g., by increasing the number of separation trains. The operability method and app can thus provide insights on how these modifications could be performed. 


\subsection{DMA-MR Application}

To select the design and the nominal operation that achieve PI targets and minimize process footprint, the NLP-based approach is applied to a $7 \times 3$ DMA-MR subsystem. The formulation $\mathrm{P} 1$ is employed for the determination of the feasible DOS (DOS*), and P2 for the selection of the best input-output point associated with the described goals. This example correspond to a modified case-study presented in ref. ${ }^{67}$, which addresses the same subsystem but with distinct objectives.

For building the $A I S \in \mathbb{R}^{7}, 4$ operational inputs and 3 design inputs are selected. Using the information from Tables 2 and 3 , the AIS is structured as follows:

$$
\begin{aligned}
& u_{\text {des }, 1} \stackrel{\text { def }}{=} \text { Reactor length }(\mathrm{cm}) \\
& u_{d e s, 2} \stackrel{\text { def }}{=} \text { Tube diameter }(\mathrm{cm}) \\
& u_{\text {des }, 3} \stackrel{\text { def }}{=} \text { Permeance }\left(\mathrm{mol} /\left(\mathrm{s} \cdot \mathrm{m}^{2} \mathrm{~atm}^{\frac{1}{4}}\right)\right) \\
& u_{\text {des }, 4} \stackrel{\text { def }}{=} \text { Selectivity }(-) \\
& u_{o p, 1} \stackrel{\text { def }}{=} \text { Methane feed }\left(\mathrm{cm}^{3} / \mathrm{min}\right) \\
& u_{o p, 2} \stackrel{\text { def }}{=} \text { Sweep gas feed }\left(\mathrm{cm}^{3} / \mathrm{min}\right) \\
& u_{o p, 3} \stackrel{\text { def }}{=} \text { Tube pressure (atm) } \\
& A I S_{\text {des }}=\left\{u_{\text {des }} \in \mathbb{R}^{4} \mid\left(10,0.5,1 \cdot 10^{-4}, 300\right) \leq u_{\text {des }} \leq\left(100,2.0,1 \cdot 10^{-2}, 1 \times 10^{5}\right)\right\} \\
& A I S_{o p}=\left\{u_{o p} \in \mathbb{R}^{3} \mid(7,9,1.00) \leq u_{o p} \leq(9,11,1.12)\right\} \\
& \text { AIS }=\left\{u \in \mathbb{R}^{7} \mid\left(u_{\text {des }}^{\min }, u_{o p}^{\min }\right) \leq u \leq\left(u_{\text {des }}^{\max }, u_{o p}^{\max }\right)\right\} \\
& \text { where }\left(u_{\text {des }}^{\min }, u_{o p}^{\max }\right)=\left(10,0.5,1 \times 10^{-4}, 300,7,9,1.00\right) \text { and }\left(u_{\text {des }}^{\max }, u_{o p}^{\max }\right)=(100,2.0,1 \times \\
& \left.10^{-2}, 1 \times 10^{5}, 9,11,1.12\right) \text {. }
\end{aligned}
$$




$$
\begin{aligned}
& y_{1} \stackrel{\text { def }}{=} \text { Benzene production }(\mathrm{mg} / \mathrm{h}) \\
& y_{2} \stackrel{\text { def }}{=} \text { Methane conversion }(\%) \\
& y_{3} \stackrel{\text { def }}{=} \text { Cost factor }(-) \\
\text { DOS }= & \left\{y \in \mathbb{R}^{3} \mid(20,35,0) \leq y \leq(25,45,100)\right\}
\end{aligned}
$$

In the Operability App, the above AIS and DOS are entered. The process model is uploaded in the form of a MATLAB script. The nonlinear objective function and the process constraints are input. To obtain elements in the DOS, a discretization is employed by informing the app of the size of the grid. For being a value that provides a good resolution, a desired grid of 10 elements in each dimension is entered in the app. As an additional configuration, the option of generating a solution inside the AIS and DOS is selected.

The operability app runs, generating the sets $\mathrm{DOS}^{*}$ and $\mathrm{DIS}^{*}$ as well as the inputoutput data point that minimizes the process footprint. Figure 31 depicts the DOS* and the selected intensified point as well as the color-coded footprint. The inputs and outputs of the selected intensified design and nominal operation correspond to $u=$ $\left(16.4,0.544,9.97 \times 10^{-3}, 2.44 \times 10^{4}, 7.40,10.95,1.002\right) \quad$ and $\quad y=(20.9,35.9,33.7)$, respectively.

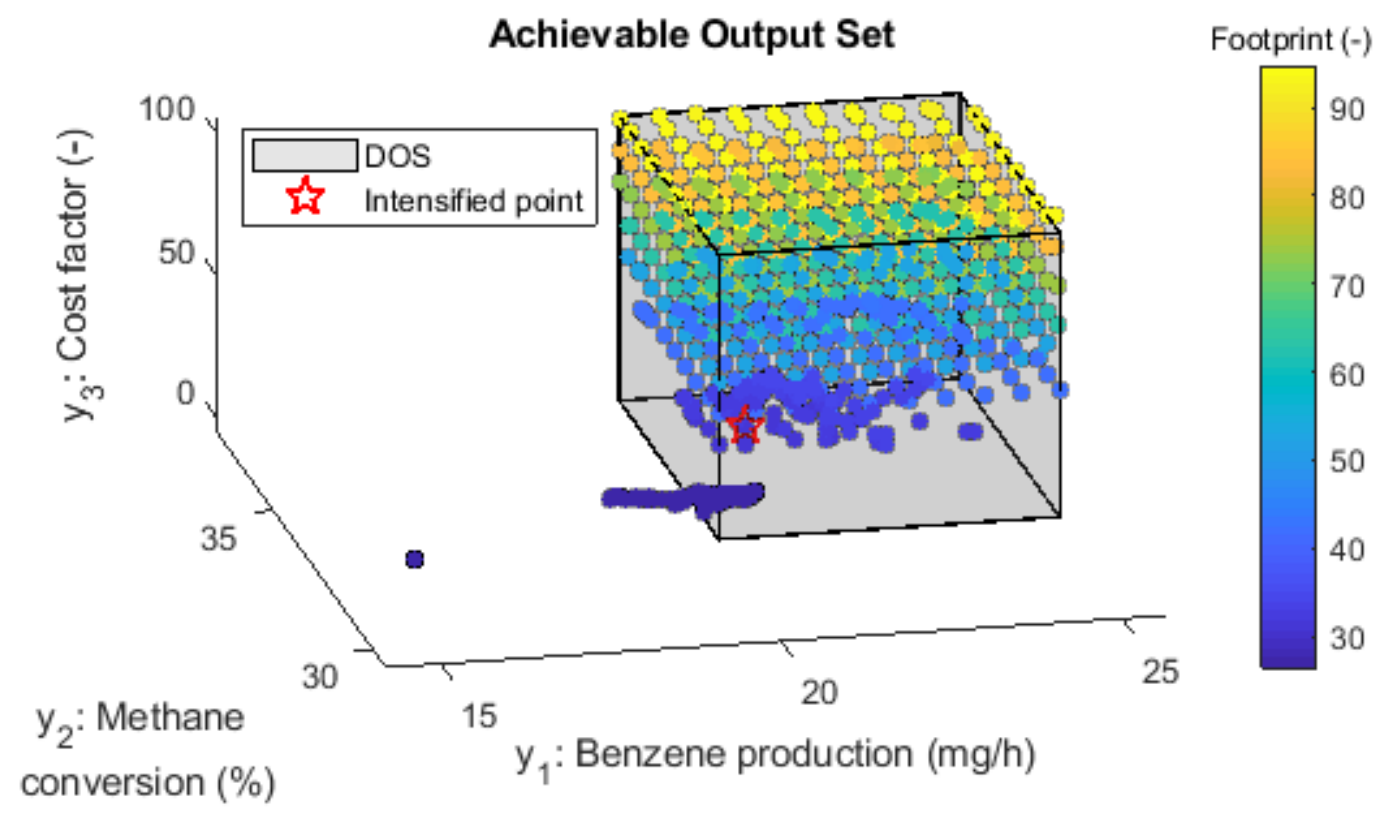

Figure 31. DOS* (color coded points) and intensified point for DMA-MR 7×3 application. 
An important observation from Figure 31 is that the complete DOS is not achievable. Combinations of high cost factor, benzene production and methane conversion can be achieved by reactors of large footprints. As the cost factor decreases, the achievability of the DOS is limited. This behavior is expected as the reduction in cost factor is associated with membranes of lower quality, less catalyst mass and smaller reactors. ${ }^{42}$ The direct relationship between cost factor and footprint derives from the fact that the size of the reactor proportionally affects its associated cost.

As a comparison with previously applied $2 \times 2$ and $3 \times 3$ similar subsystems that employed the same objectives, one can notice that the obtained modular design is even smaller. For both previous cases, reported values of reactor length and tube diameter consisted of about $17 \mathrm{~cm}$ and $0.57 \mathrm{~cm} .5,17$ The further reduction in size here is mainly due to the inclusion of operational variables, which present contrasting values with the nominal operation of previously addressed applications, fixed at $8 \mathrm{~cm} / / \mathrm{min}$ for methane feed, $10 \mathrm{~cm}^{3} / \mathrm{min}$ for sweep gas feed and 1 atm of tube pressure. 


\section{Chapter 7 Modular Cost and Operability Analysis for Technology Deployment}

This chapter addresses cost and operability challenges that are present in the technology deployment of intensified modular plants. A systematic TEA framework for modular systems is proposed for cost estimation and profitability with respect to conventional technologies. Classic concepts of engineering economic analysis of chemical processes are extended to include estimation of capital and operating costs of intensified modular plants based on available literature data. ${ }^{68}$ Process operability analysis is used to overcome operational challenges that may arise when laboratory designs are scaled up to commercial modular scale.

The concept of economy of learning is newly introduced to the cost analysis of modular systems. The economy of learning is based on experience curves that account for changes in purchase cost according to the number of manufactured units. Here, cost estimations are divided into two scenarios according to experience curve models: (i) the experience curve is modeled based on previous modular deployment data; or (ii) the experience curve parameters are varied to verify if distinct profitability targets can be achieved. The examination of the two cases provides insights on competitiveness and determines the situations in which the candidate modular technology is promising for future deployment or further research.

An application to a modular and intensified hydrogen production unit is considered. Process flowsheet synthesis is carried out around an intensified microchannel reactor for steam methane reforming (SMR), which consists of an integrated alternative to the conventional unit operations of pre-reforming, reforming furnace, and high-temperature shift reactors. Operability analysis is employed to study feasible process operating regions and determine the nominal modular operation. ${ }^{2,4}$

Then, TEA of the hydrogen unit is performed in comparison with an adopted conventional SMR plant. Measures of scaled equivalent annual operating cost (EAOC) are used to assess how competitive modular units are, independent of size and production capacity. A supplementary investigation is also presented supposing bulk, or massive, purchase of modular units to attain the same hydrogen production as the 
conventional plant. Another operability study is carried out to determine small design adjustments, and check flexibility of the developed modular unit with respect to disturbances on NG composition.

In this chapter, the economy of learning model is introduced at first. Then, the modular TEA framework is discussed, followed by the modular hydrogen unit application, which comprises cost and operability analysis.

\subsection{Economy of Learning}

Economies of learning rely on the concept of experience curves to account for the effects of the level of maturity on the cost of the manufactured technology. The economy of learning is also known as the economy of mass production and the economy of numbers because it considers the mass production behavior in manufacturing. Unit prices tend to decrease with expansion in cumulative production output due to continuous manufacturing improvements. ${ }^{69}$

The experience curve is a generalization of the learning curve concept. Learning curves are associated with reductions in cost of labor due to gained labor skills over worked hours. Experience curves consider overall cost savings due to improvement in various organizational routines including individual skills, manufacturing techniques, innovation, etc. While the learning curve is generally used to forecast labor costs, the experience curve is a strategic tool to manage cost reductions as units are manufactured..$^{52}$ Several shapes have been proposed for the experience curve. ${ }^{53}$ For example, the power law function has been used to represent the experience curve. Here, the power law function is adapted to explicitly include a plateau effect as follows: ${ }^{54}$

$$
\begin{gathered}
F_{n}=1-R_{n}=\left\{\begin{array}{l}
n^{-\alpha}, \quad \text { if } n^{-\alpha} \geq R_{\max } \\
R_{\max }, \quad \text { if } n^{-\alpha}<R_{\max }
\end{array}\right. \\
C_{n}=F_{n} \cdot C_{1}
\end{gathered}
$$

where $F_{n}$ is a purchase cost factor that represents the reduction in purchase cost, $R_{n}$ is the reduction in purchase cost, $n \in \mathbb{N}$ is the number of manufactured units, $\alpha$ is an experience rate exponent, $R_{\max }$ is the maximum reduction in purchase cost, and $C_{n}$ is the 
purchase cost of the $\mathrm{n}^{\text {th }}$ manufactured unit. $R_{\max }$ corresponds to a plateau in the experience curve, in which the technology achieves maturity. Note that the purchase cost factor $F_{n}$ is a multiplier that indicates no cost reduction for the first-of-a-kind unit, i.e., $F_{1}=$ 1 or $100 \%$ of initial cost and $R_{1}=0$ or $0 \%$ reduction.

Figure 32 illustrates the experience curve for a situation in which a maximum reduction in cost of $R_{\max }=30 \%$ and an experience rate exponent $\alpha=0.15$ are assumed. In this example, the experience curve plateau and technology maturity happen around the 11 th unit.

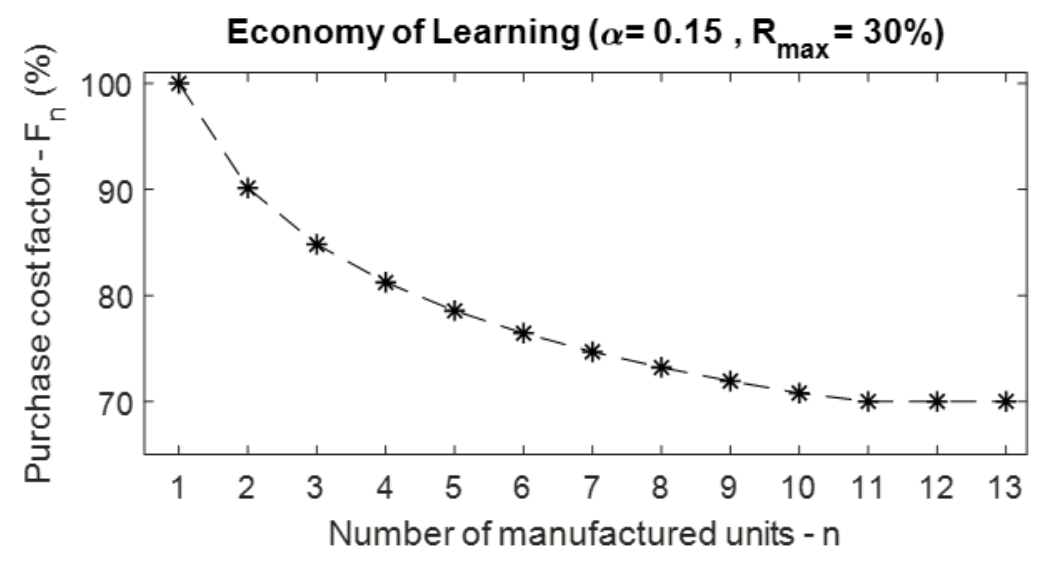

Figure 32. Illustration of the experience curve considering $\alpha=0.15$ and $R_{\max }=30 \%$.

In this work, $\alpha, R_{\max }$ and $n$ are referred to as experience parameters. The values of $\alpha$ and $R_{\max }$ are initially fitted to literature values of $F_{n}$ for evaluation of a base case scenario. Then, through further studies, the experience curve is shaped for competitiveness with respect to benchmark technologies.

\subsection{Modular Techno-Economic Analysis Framework}

This framework extends existing cost correlations to include intensified modular systems. At first, a compatible conventional technology is adopted as benchmark for cost comparison. Then, modular and conventional process flowsheet specifications are considered, and estimations of capital and operating costs of both technologies are performed. Adaptation of traditional costing methods are developed for the modular 
technology so that reference values associated with conventional plants can be employed with the economy of learning.

The cost estimation is divided into two scenarios: (i) a base case scenario, in which experience parameters are estimated using literature data; and (ii) profitability scenarios, in which experience parameters are varied together with other significant variables to achieve break-even and further cost reductions. The division into these scenarios is motivated by the fact that the experience curve should be managed as pointed out above. Therefore, the base case scenario is used for an initial cost performance analysis that determines if the economy of learning and other parameters should be considered for profitability. In case the unit price reduction due to economy of learning is significant for total cost, the profitability scenarios investigate which learning behavior the modular manufacturing should present to be competitive with respect to the conventional technology.

The base case and the profitability scenarios are bridged by a sensitivity analysis step. Conclusions from the initial cost performance analysis of the base case are used to determine which promising variables should be further analyzed. The sensitivity analysis screens these variables and checks which ones should be considered in the profitability scenarios.

The steps of the developed modular TEA framework are briefly summarized in Figure 33. The requirements of this framework are knowledge about nominal operating points and process flow diagram topologies for the technologies to be compared. The application of these steps is recommended for analysis of modular systems that have undergone process synthesis and, if applicable, process operability analysis. Each step is discussed in detail in the following subsections. Subscripts con, mod and MP are associated with conventional plant, modular units and assembled modular plant, respectively. 


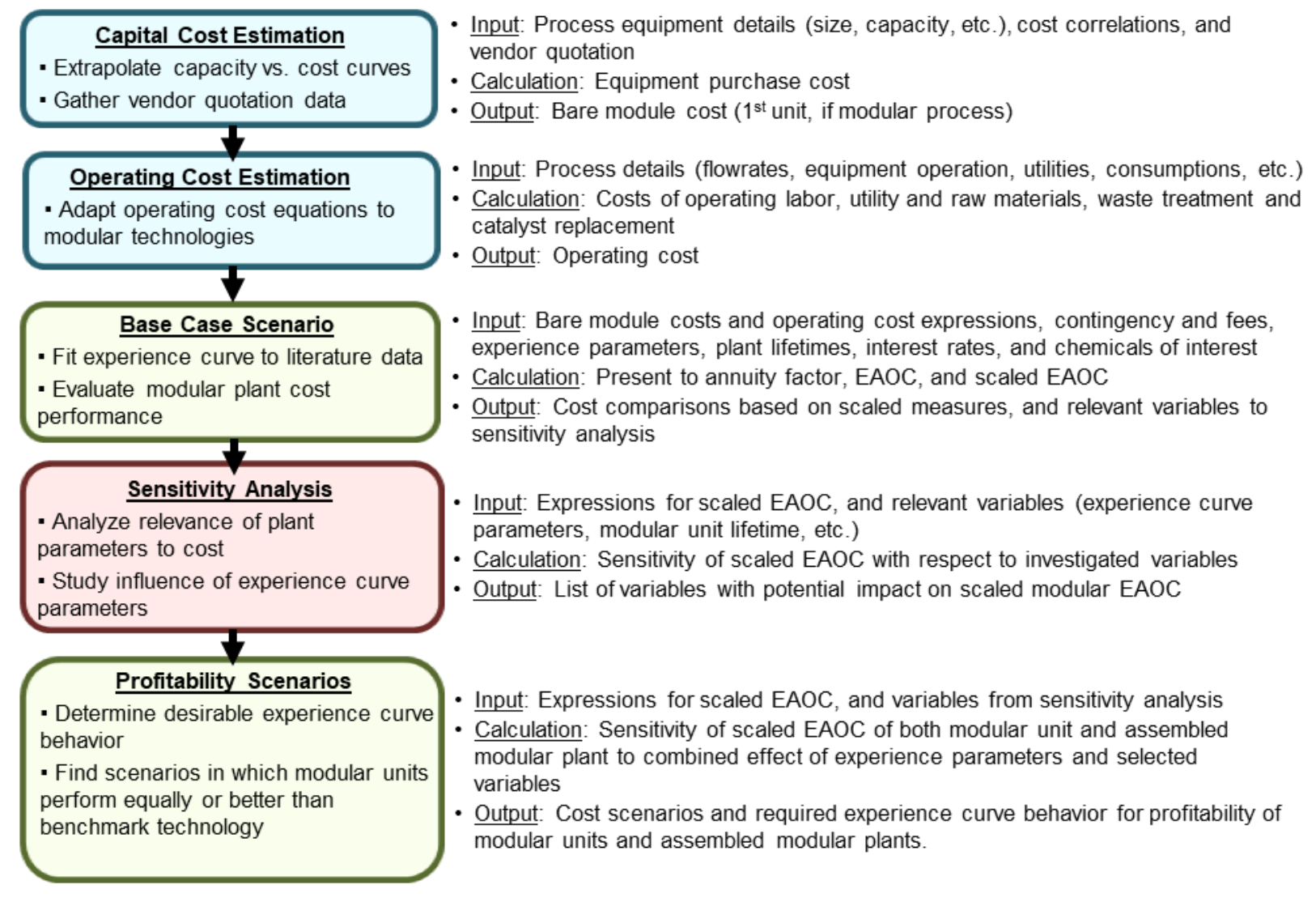

Figure 33. Summarized step-by-step modular TEA framework.

\subsubsection{Capital Cost Estimation}

In general, capital costs can be estimated by first calculating purchase cost at base conditions, followed by cost additions due to custom materials and operation, and finally by considering indirect costs, contingency, fees, and the presence of auxiliary facilities. The purchase cost is usually determined using process capacities (volume, diameter, area, power, flowrates, etc.), which are either associated with capacity vs. cost data or with the six-tenths rule. ${ }^{68}$ Here, these methods are applied to estimate the capital cost of conventional and modular technologies. New adaptations are developed to extend the estimation methods to modular processes.

The modular equipment is approximated to the closest possible conventional equipment type. Then, the purchase cost calculation is performed by extrapolating capacity vs. cost curves beyond minimum reported sizes. The consideration of custom 
conditions and other costs factors is replicated from the methodology for costing regular plants. While an estimation of modular capital cost is allowed, this method provides an upper bound price estimate, as the economy of scale benefits from large equipment capacities, and the modular construction projects are expected to be faster, safer and cheaper. ${ }^{41}$

Alternatively, quotation data for modular technologies is recommended when available. Nevertheless, the generation of capacity vs. cost data is hindered due to the degree of customization of new modular technologies. As a solution, when single cost data points are available, the six-tenth rule can be applied.

Bare module costs are defined as capital costs that include purchase cost and all direct and indirect costs, but contingency and fees. These values are estimated for both conventional and modular processes. When calculated, the conventional process presents a final value, represented by $C_{B M, c o n}$, because the economy of learning is reserved to the production of modular systems. The bare module cost estimation of the modular process, represented by $C_{B M, \bmod }$, is in turn associated only with the cost of the first produced modular unit. In this work, the cost of $\mathrm{n}^{\text {th }}$ manufactured modules is indicated by superscripts, and, therefore, the first modular unit presents $C_{B M, \bmod }^{1}$.

For subsequent modular units, assuming an experience curve described by Equation (70), the bare module cost can be estimated according to the level of maturity. In this case, Equation (72) is the result of adapting Equation (71) to the modular bare module cost case.

$$
C_{B M, \text { mod }}^{n}=F_{n} \cdot C_{B M, \text { mod }}^{1}=\left(1-R_{n}\right) \cdot C_{B M, \text { mod }}^{1}
$$

where $C_{B M, m o d}^{n}$ is the bare module cost of the $\mathrm{n}^{\text {th }}$ unit, $F_{n}$ is the multiplier for cost reduction for the $\mathrm{n}^{\text {th }}$ modular unit, $R_{n}$ is the actual cost reduction, and $C_{B M \text {,mod }}^{1}$ is the bare module cost of the first modular unit estimated above.

Another important consideration is related to values of contingency and fees. Typically, contingency and fees are lumped together and add about $3 \%$ and $15 \%$ to the total capital cost, respectively. Contingency represents reliability in cost data and completeness of the flowsheet. ${ }^{68}$ In this framework, the sum of $18 \%$ for contingency and 
fees is assumed for conventional technologies as cost data is likely to be available. For modular systems, cost data is notably scarce, and, therefore, the usual value of contingency of $15 \%$ may not be realistic. Therefore, values of contingency above $15 \%$ are considered for modular cases. Equation (73) shows the total module cost, or fixed capital investment, calculated as a function of bare module and contingency and fees. If desired, new site development could be included by adopting the measure of grassroots roots cost, which corresponds to an approximate increase of $50 \%$ to the bare module cost.

$$
C_{T M}=(1+a) \cdot C_{B M}
$$

where $C_{T M}$ is the total module cost, $a$ represents additional cost increase due to contingency and fees, and $C_{B M}$ is the bare module cost.

In this step, the two values of bare module costs associated with the conventional process and first modular unit are calculated. Total module costs are estimated in the base case scenario, sensitivity analysis, and profitability scenarios, where experience parameters and values of contingency and fees are analyzed in more depth.

\subsubsection{Operating Cost Estimation}

The operating cost includes direct costs, fixed costs, and general expenses. A detailed explanation of each of these terms correspond to the definition of "manufacturing costs" for traditional chemical process design costing from ref. ${ }^{68}$. Here, the term "operating cost" is chosen to establish distinction from the costs related to the manufacturing, or fabrication, of modular units. To systematically estimate the operating costs, the classification of operating cost as direct and indirect costs and general expenses is employed. These costs are in turn broken down as functions of the depreciation, fixed capital investment, operating labor, utilities, waste treatment and raw materials. ${ }^{68}$ Here, conventional operating cost equations are adapted for an adequate comparison between modular and conventional technologies.

For process integrated modular systems, straightforward distinction between raw materials and utilities may be impaired. New modular technologies are also likely to count 
on advances in the field of novel catalysts, thereby making catalyst replacement play an important role in the modular operating cost. To account for these particularities, the operating cost equation from ref. ${ }^{68}$ is modified. Raw materials and utilities are lumped in a single cost term, and the catalyst replacement cost is explicitly included as part of direct manufacturing costs. As a result, Equation (74) describes the estimated annual cost of operation as follows.

$$
C O M=0.280 \cdot C_{T M}+2.73 \cdot C_{O L}+1.23 \cdot\left(C_{U R M}+C_{W T}+C_{C A T}\right)
$$

where $C O M$ is the total operating cost; $C_{T M}$ is the total module cost; $C_{O L}$ is the cost of operating labor; $C_{U R M}$ refers to costs of utilities and raw materials; $C_{W T}$ is the cost of waste treatment; and $C_{C A T}$ is the cost of catalyst replacement. A depreciation of $10 \%$ of fixed capital investment was assumed.

For both modular and conventional technologies, total module costs are calculated using obtained values of bare module cost from the previous step and Equation (73). Here, the cost of operating labor is assumed to be the same as in a regular plant of similar scale. First, the cost of operating labor is estimated for the conventional technology, then it is linearly scaled for the modular unit using production capacities. Equation (75) corresponds to the estimation of modular operating cost.

$$
C_{O L, \text { mod }}=\frac{T_{i, \text { mod }}}{T_{i, \text { con }}} \cdot C_{O L, \text { con }}
$$

where $C_{O L}$ refers to the costs of operating labor, $T_{i, c o n}$ and $T_{i, m o d}$ correspond to productions of a chemical of interest of the conventional plant and modular unit respectively. Note that the above assumption also provides an upper bound price estimate, since modular systems are expected to be more autonomous and less staffed. ${ }^{41}$ This assumption allows the estimation of operating cost, as there is a lack of estimation methods for costing modular operating labor in the available literature.

Stream flowrates of utilities, waste treatment and raw materials are determined using process simulation if plant data is not available. The cost of raw materials is estimated using historical market values and projections. The costs of utilities and waste treatment are calculated either: (i) using reference tables, assuming utility generation and 
waste treatment happen as in large-scale industries; or (ii) by synthesizing and costing modular equipment that perform those tasks.

Utilities bought at the plant's boundary limits such as water, air and power are likely not to be affected by modularization. However, generated utilities and treatments that require substations depends on the modularity considerations. The inclusion of modular substations results in a cost that is based on utilities (or raw materials) bought at the boundary limits, rather than a cost representation based on reference tables.

The outcomes of this step are the expressions for $C O M_{\text {con }}$ and $C O M_{\text {mod }}$ as functions of bare module cost, contingency, and, for the modular case, experience parameters and number of manufactured modules. The dependency of the operating cost functions with respect to these variables can be expressed as $\operatorname{COM}_{\text {con }}\left(C_{T M, c o n}\right)=$ $\operatorname{COM}_{\text {con }}\left(C_{B M, \text { con }}, a_{\text {con }}\right)$ and $\operatorname{COM}_{\text {mod }}\left(C_{T M, \text { mod }}\right)=\operatorname{COM}_{\text {mod }}\left(C_{B M, \text { mod }}^{1}, a_{\text {mod }}, \alpha, R_{\text {max }}, n\right)$.

\subsubsection{Base Case Scenario Cost Estimation}

In this step, a base case scenario provides insights about the overall cost performance of the analyzed modular system in comparison with a benchmark technology of traditional scale. The objective of this study is to identify which influencing cost parameters should be further investigated using sensitivity analysis. Particularly, the relevance of the economy of learning is evidenced, indicating whether experience parameters should be further studied as well.

At first, experience parameters are estimated using literature data so that both $C O M_{\text {con }}$ and $C O M_{\text {mod }}$ can be estimated. Then, capital and operating costs are merged in a single EAOC profitability indicator. Equation (76) below depicts the calculation of EAOC by converting the capital costs to annuity. ${ }^{68}$

$$
E A O C=C_{T M} \cdot P A+C O M
$$

where $E A O C$ is the equivalent annual operating cost, $C_{T M}$ and $C O M$ follow the previous descriptions, and $P A$ is the present to annuity factor. $P A$ is a function of the plant lifetime and interest rate. Equation (77) shows how $P A$ can be calculated. 


$$
P A=\frac{i r(1+i r)^{l f}}{(1+i r)^{l f}-1}
$$

where ir is the interest rate and $l f$ is the plant lifetime.

The $E A O C$ is also scaled with respect to production of a chemical of interest so that the comparison can be performed independently of production scale. Equation (78) scales the $E A O C$ with respect to annual production of the chosen chemical.

$$
E A O C^{\prime}=\frac{E A O C}{T_{i} \cdot O P}
$$

where $E A O C^{\prime}$ is the scaled $E A O C$ in US $\$ / \mathrm{kg}$ of chemical of interest, $T_{i}$ refers to productions of a chemical of interest in $\mathrm{kg} / \mathrm{h}$ and $O P$ is the annual plant operating time in $\mathrm{h} / \mathrm{yr}$.

Finally, the scaled cost estimates are directly compared for the selection of variables that will be further investigated. The value of $E A O C^{\prime}$ indicates which technology is more profitable given the employed parameters. Specific terms are scaled and compared to show possible bottlenecks of the modular technology. For example, cost of waste treatment can be scaled using $C_{W T} / T_{i} \cdot O P$ to show how significant this cost is for composition of the $E A O C^{\prime}$.

\subsubsection{Sensitivity Analysis}

Screening of variables with a focus on economy of learning is performed through sensitivity analysis. Parameters that are uncertain in modular deployment projects are investigated, including contingency, experience parameters and modular unit lifetime. Other relevant cost variables may be included in case the cost performance from the previous step indicates potential. This step provides guidelines on which variables should be studied to find scenarios that are favorable to the modular technologies.

\subsubsection{Profitability Scenarios}

In this study, profitability is analyzed in two distinct cases: (i) a modular plant is constructed assuming fixed capital and operating costs, and the technology is evaluated at maturity; and/or (ii) a modular plant is scaled-up supposing bulk purchase of modular 
units at learning stage, and, therefore, consider a gradual decrease in costs due to the economy of learning. The first case indicates future competitiveness of the modular technology, whereas the second case analyzes profitability of the complete construction process, including higher initial costs of the first units.

This step consists of an extension of sensitivity analysis to check in which conditions modular systems may present advantages over the conventional plant. As outcome, the first case shows what conditions of cost reduction are needed for competitiveness, independently of the experience rate exponent. The second case shows how the experience rate exponent can be included in the analysis by adopting a measure of profitability that comprises deployment and operation of several modular units that compose a modular plant with production rates equivalent to the conventional scale.

\subsection{Application Process: Modular SMR}

A modular hydrogen unit is considered for the application of the developed framework. Intensification strategies are employed to provide enhanced heat transfer to the endothermic steam reforming reactions. The process model includes pretreatment, purification and generation of power and steam. The modular unit operations are integrated to minimize consumption of utilities, aiming system self-sufficiency.

If deployed, modular SMR units could be applied to monetize unused NG resources. Shale gas consists of a geologically distributed NG source that can be recovered in short term wells, and therefore require dynamic drilling and production. Landfill and associated gas in oil recovery are other examples of stranded NG, which are usually flared due to the lack of infrastructure for processing and utilization. NG in remote locations is another example of unrecovered gas linked to costly transportation and associated construction work (pipelines, etc.).

Transportability and cheaper maintenance costs are promising features to address the challenges of untapped NG. Intensified modular systems can also significantly decrease process footprint, reducing the requirements associated with groundwork and infrastructure. 
The proposed framework is applied to systematically evaluate economic feasibility of the considered modular hydrogen unit. Modular profitability is studied with a focus on the comparison with existing large-scale SMR plants. Process development and multimodel operability analysis are presented, followed by the application of the modular TEA framework and operability for flexibility analysis.

\subsubsection{Process Development}

The synthesis of the modular hydrogen unit starts with equipment that represent the most drastic changes. The remaining parts of the process are then designed by creating modular versions of the necessary unit operations to complete the flowsheet synthesis.

Initially, a microchannel reactor is considered as an intensified modular equipment that substitutes the conventional unit operations of pre-reforming, reforming furnace, and high-temperature shift. The microchannel reactor is scaled up from literature experimental data. ${ }^{70}$ Figure 34 depicts a simplified scheme corresponding to the literature experimental design. ${ }^{70}$

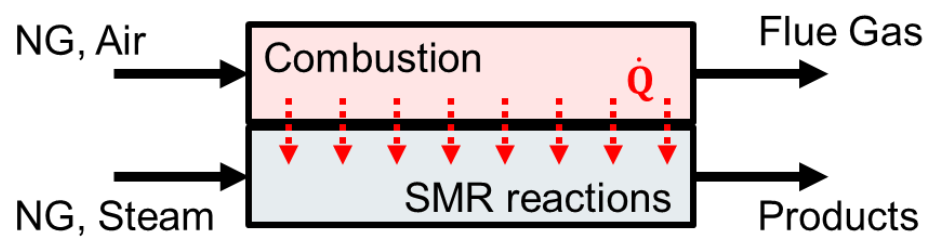

Figure 34. Experimental scheme of consecutive microchannels.

NG undergoes combustion in the combustion microchannel. Then, the increased contact area between microchannels is responsible for enhanced heat transfer from the combustion microchannel to a reaction microchannel, where hydrogen production is facilitated by endothermic reactions. Particularly, the following reactions take place in the reaction microchannel:

$$
\begin{gathered}
\mathrm{CH}_{4}+\mathrm{H}_{2} \mathrm{O} \leftrightarrow \mathrm{CO}+3 \mathrm{H}_{2} \\
\mathrm{CO}+\mathrm{H}_{2} \mathrm{O} \leftrightarrow \mathrm{CO}_{2}+\mathrm{H}_{2}
\end{gathered}
$$




$$
\mathrm{CH}_{4}+2 \mathrm{H}_{2} \mathrm{O} \leftrightarrow \mathrm{CO}_{2}+4 \mathrm{H}_{2}
$$

Equations (79) to (81) represent steam-methane reforming, water-gas shift and reverse methanation, respectively. The microchannels are filled with specific types of catalyst. Details on catalyst distribution and geometry of microchannels can be found in ref. ${ }^{70}$.

Scale up is carried out for the design of a microchannel reactor using experimental data. As a result, about 3,000 paired microchannels compose the design of a reactor that produces approximately $0.36 \mathrm{Sm}^{3} / \mathrm{s}$ of hydrogen corresponding to expected commercial performances. ${ }^{70}$ Heat losses drop from $45 \%$ at experimental scale to less than $5 \%$ for the commercial scale design, as consequence of placing a high number of microchannels together in an optimal manner. ${ }^{70}$ Subsection 7.3.2 shows how operability analysis is employed to adjust the ratio between fuel and combustion fed to the system, resulting in a new nominal process design and operation. Subsection 7.3.4 verifies possible design modifications and how flexible the developed process is with respect to changes in NG composition.

Process synthesis is then performed to include unit operations of pretreatment of NG, steam production and hydrogen separation and purification. The NG passes by desulphurization beds, where sulfur removal takes place. Then, it is mixed with steam, and converted to a mixture of hydrogen, carbon monoxide, carbon dioxide, steam, and unconverted methane in the microchannel reactor. Water is knocked out from the product stream and treated for reuse in the steam production. Pressure swing adsorption (PSA) finalizes the process by separating about $90 \%$ of all produced hydrogen. Figure 35 shows a simplified block flow diagram of the proposed modular hydrogen unit.

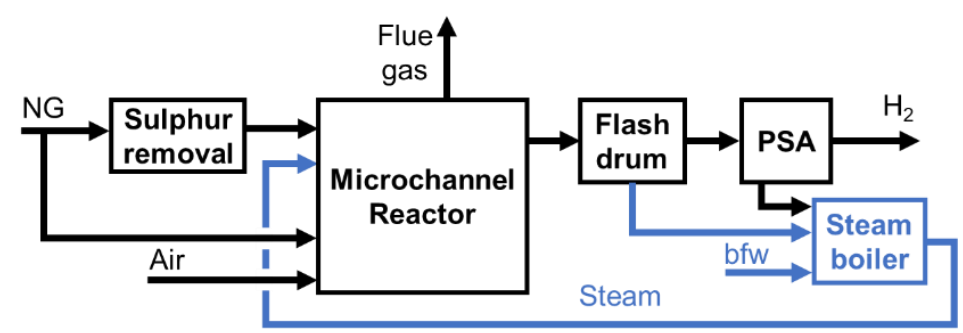

Figure 35. Simplified block flow diagram: modular hydrogen unit. 
The modular SMR process is modeled in Aspen Plus® Version 9.0. For the microchannel reactor model, combustion is employed in a stoichiometric reactor and reaction in a plug-flow reactor. Multipliers account for scaling-up and heat transfer follow a custom model in which a fraction of the produced heat is dissipated, and the remaining heat is transferred to the reaction microchannel.

Utility heat exchangers are initially placed for conditioning streams. Aspen Process Economic Analyzer is employed to integrate the heat exchangers and minimize utility usage. Figure 36 shows the final hydrogen unit process model in Aspen Plus. Details on model assumptions and specific flowrates can be found in Appendix A.

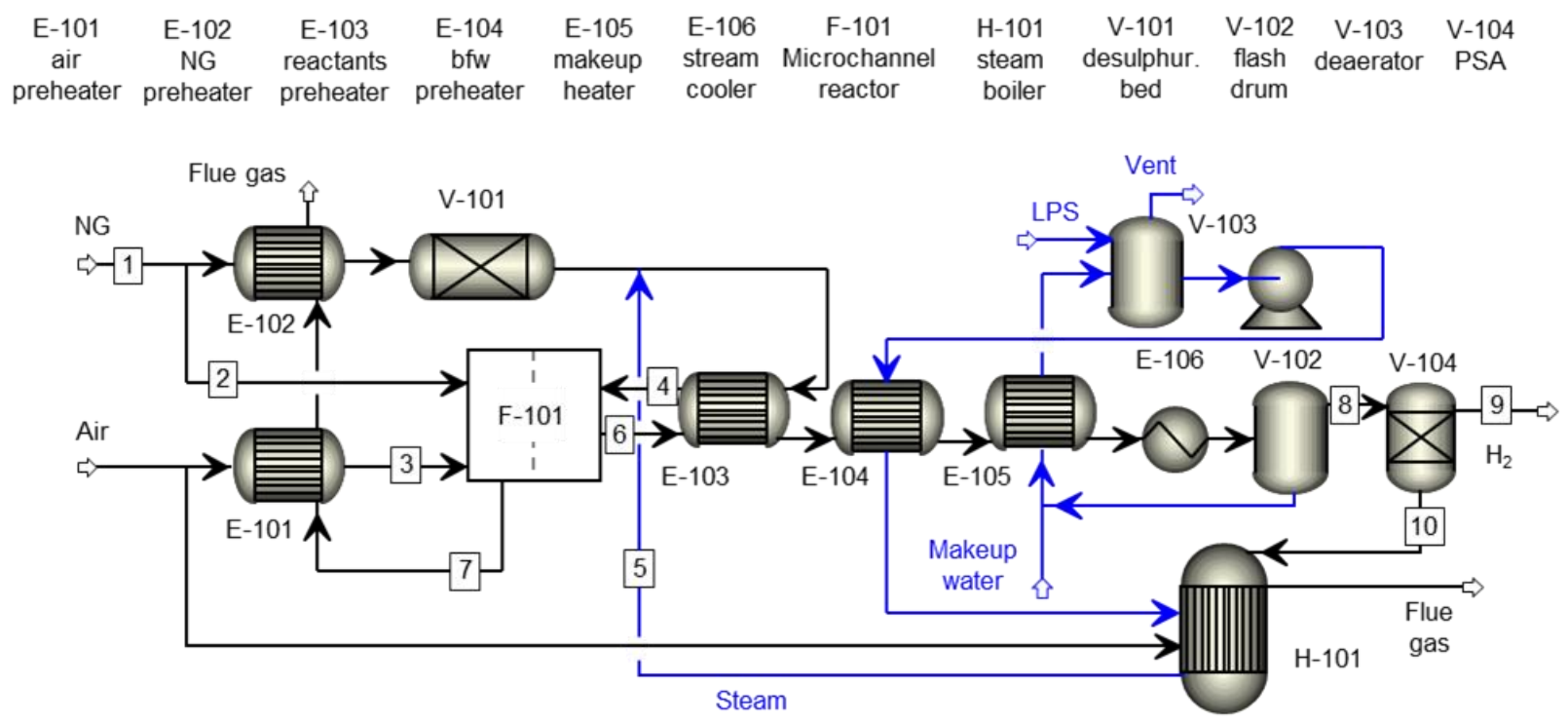

Figure 36. Process flow diagram: modular hydrogen unit (adapted from Aspen Plus).

\subsubsection{Selection of Nominal Design and Operation}

The experimental design of the microchannel reactor operates in accordance with a heat loss of about $45 \%$ as pointed out in Subsection 7.3.1. When scale up is completed, and the commercial scale design presents a heat loss of less than $5 \%$, the heat production in the combustion microchannels must be regulated. Process operability analysis concepts are employed to determine a feasible microchannel reactor operation in which heat is generated according to reaction needs. ${ }^{2,4}$ The flowrates of NG as fuel, NG as feedstock 
and steam are systematically changed to regulate the production of heat and the consumption by endothermic reaction. Although the operability analysis focuses on the operation of the microchannel reactor, input and output variables associated with the entire unit are selected.

The described process model is integrated with MATLAB for the analysis of process operation. ActiveX automation server is used to allow a MATLAB script to open, change variables, simulate, collect results, and register logs from the Aspen Plus simulations. For this application, the selected MVs are the NG flowrate directed to reaction microchannels, NG flowrate directed to combustion microchannels and steam to carbon ratio. The $\mathrm{CV}$ s are methane conversion, hydrogen flowrate to unit's boundary limit $(B L)$ and required heat efficiency. A MATLAB script is written to change MVs and collect CVs after the simulations are run.

The expected ranges in which MVs are changed compose the AISop. The simulations of all operation points define the AOS. The ranges of NG flowrate sent to reaction microchannels and steam to carbon ratio are based on reported values from the experimental microchannels. ${ }^{70}$ The range of NG flowrate employed as fuel is determined by adopting a typical experimental value as upper bound and a value close to zero as lower bound since fuel usage decreases with the scale up due to low heat losses.

The desired ranges of CVs describe the DOS. Methane conversion experimental values are assumed as the lower limit and the maximum mathematically possible conversion (100\%) as the upper limit. Similarly, desired amounts of produced hydrogen have their lower and upper limits determined by reported experimental performance and maximum production assuming full methane conversion. The required heat efficiency is defined as the complement of heat loss, and, as the heat loss is expected to be lower than $5 \%$, the required heat efficiency should be above 95\%. Tables 6 and 7 contain the adopted ranges for AISop and DOS sets. 
Table 6. Operational input variables and available ranges.

\begin{tabular}{ll}
\hline operational input variable & available range \\
\hline \hline NG - SMR $(\mathrm{kmol} / \mathrm{h})$ & $16.46-17.24$ \\
$\mathrm{NG}$ - fuel (kmol/h) & $0.50-9.52$ \\
Steam to carbon ratio (-) & $3.0-4.8$ \\
\hline
\end{tabular}

Table 7. Output variables and desired ranges.

\begin{tabular}{ll}
\hline output variable & desired range \\
\hline $\mathrm{CH}_{4}$ conversion (\%) & $92.48-100$ \\
Hydrogen to $\mathrm{BL}\left(\mathrm{Sm}^{3} / \mathrm{s}\right)$ & $0.32-0.41$ \\
Required heat efficiency (\%) & $95-100$ \\
\hline
\end{tabular}

The composition of NG is considered as a system disturbance. For the selection of nominal design and operation, NG is assumed to be at a nominal point, i.e., there is no perturbation and therefore $E D S=d^{N}$. An average between the methane concentration of 95.8\% from the experimental microchannel design and typical methane concentrations of at least $99.3 \%$ for pipeline quality is adopted. ${ }^{70,71}$ Therefore, the nominal NG composition is defined as $d_{1}^{N}=97.56 \%$, in which $d_{1}$ is the methane concentration in $\mathrm{mol}_{\mathrm{CH}_{4}} / \mathrm{mol}_{\mathrm{NG}}$.

The inverse multimodel algorithms from Subsection 3.2.1 are run to find the combinations of MVs that achieve the described DOS. Particularly, multimodel algorithms include interpolations to better define the resulting regions. Figure 37 shows the AIS op and calculated feasible operating region. Figure 38 shows the AOS, the DOS and their intersection, which determines the feasible operating region. 


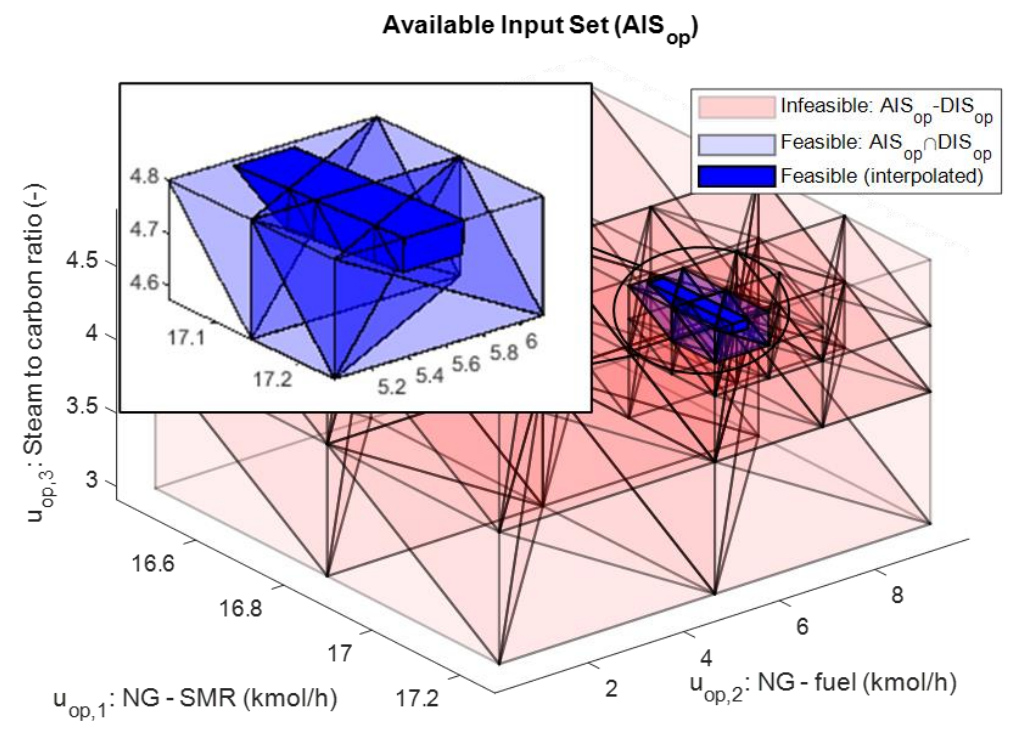

Figure 37. AISop and feasible operating input region.

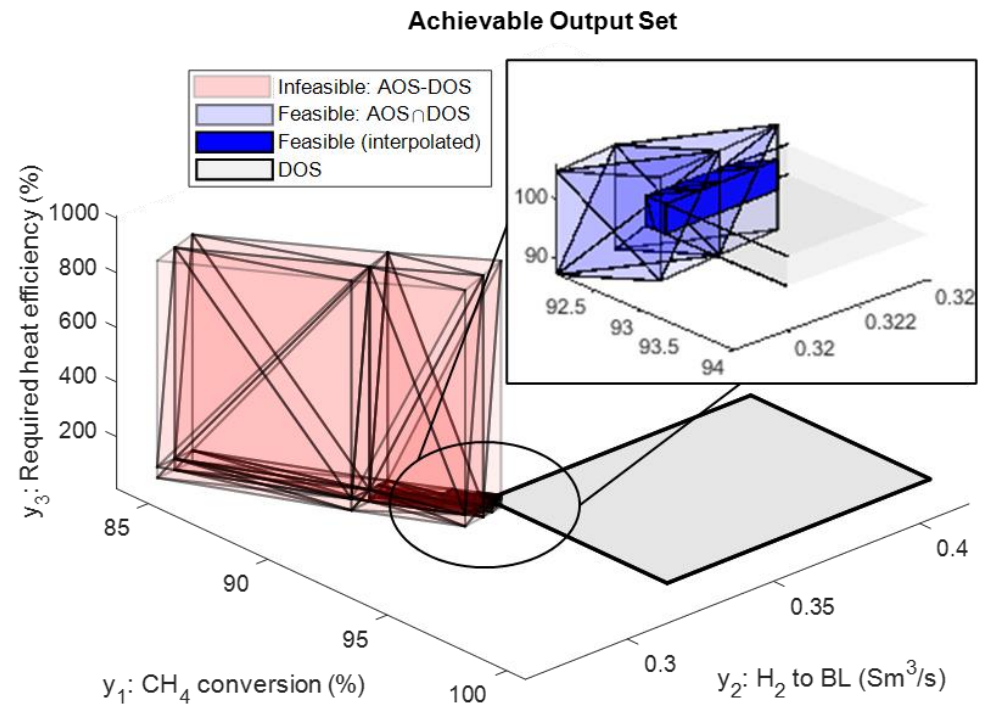

Figure 38. AOS, DOS and feasible operating output region.

The feasible operating region is approximated by parallelepipeds and the nominal operation is assumed to be the point in the center of that region. The selected nominal operation is defined by NG flowrates to reaction and combustion microchannels of $u_{o p, 1}^{N}=$ $17.15 \mathrm{kmol} / \mathrm{h}$ and $u_{o p, 2}^{N}=5.52 \mathrm{kmol} / \mathrm{h}$, respectively, and steam to carbon ratio of $u_{o p .3}^{N}=$ 
4.76. This nominal point takes the CVs to assume the values of $y_{1}^{N}=92.57 \%$ for methane conversion, $y_{2}^{N}=0.32 \mathrm{Sm}^{3} / \mathrm{s}$ for final hydrogen production, and $y_{3}^{N}=96.56 \%$ for required heat efficiency. Note that the chosen MVs take the CVs inside the designed DOS.

Finally, using the nominal point, the entire plant is checked for possible constraint violations and compliance with specified temperatures. Heat exchanger areas are obtained using design mode of Aspen Plus and nominal operation. Subsection 7.3.3.1 contains design specification of the developed process. In Subsection 7.3.4, the operability analysis is complemented with a flexibility study, considering disturbances in methane composition and achievability of the nominal operation. Minor design changes are also considered for improvement of system flexibility.

\subsubsection{Application of Modular Techno-Economic Analysis Framework}

A conventional SMR hydrogen plant that produces $29.5 \mathrm{Sm}^{3} / \mathrm{s}$ of hydrogen is adopted as a benchmark technology for the application of the modular TEA framework. The conventional SMR presents regular equipment sizes and noticeable differences to the modular unit. Besides converting methane through several unit operations, a reactive furnace provides excess heat that is used for steam production. The considered SMR is based on ref. ${ }^{72}$, where specific details regarding the process flow diagram and flowrates can be found. Figure 39 shows the simplified blow flow diagram of the conventional SMR.

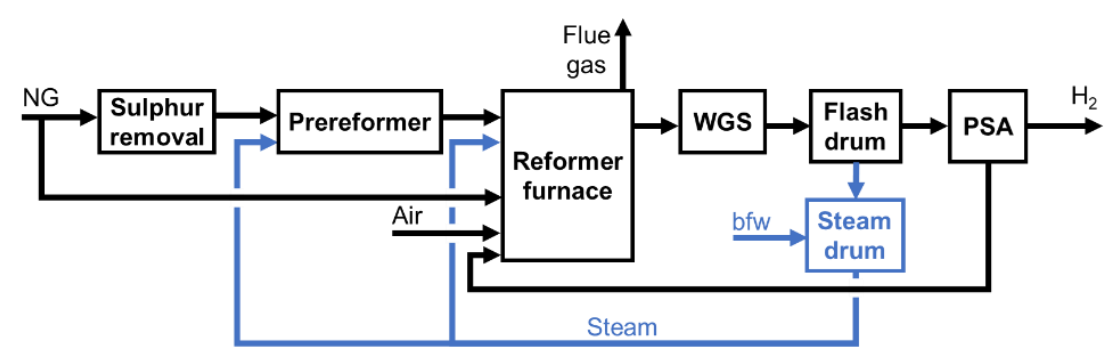

Figure 39. Simplified block flow diagram: conventional SMR plant.

Considering the obtained modular unit and the adopted conventional counterpart, the same steps from Subsection 7.2 are applied in the following subsections. For fairness, 
cost techniques and assumptions are performed on both modular and conventional systems whenever possible.

\subsubsection{Capital Cost Estimation}

For the considered benchmark SMR plant, capital cost is obtained through adjustments using reported values. ${ }^{72}$ After inclusion of location factor and currency adjustments, the bare module cost of the considered SMR plant is about $C_{B M, c o n}=\mathrm{US} \$ 78,300,000 .^{73}$

The capital cost of the modular hydrogen unit is estimated by combining the two techniques described in Subsection 7.2.1. For most modular equipment, the capital cost is obtained from conventional equipment approximation and extrapolation of capacity vs. cost curves. For equipment associated with PSA, desulfurization and deaeration, modular processes from the literature are scaled using the six-tenths rule.

For the approximation of modular equipment as conventional, all the heat exchangers are considered as shell and tube of floating head type; the microchannel reactor as a reformer furnace; the boiler as a conventional steam boiler; and the flash drum as a vessel. To extrapolate the capacity vs. cost curves associated with each type of equipment, the methodology from ref. ${ }^{68}$ is repeated, but allowing the estimation of purchase cost to be calculated outside of expected limits when needed. Factors associated with design pressure, materials of construction and other indirect costs are included to convert the purchase cost estimation into bare module cost estimations.

The PSA and desulfurization beds have their purchase cost scaled using modular equipment literature data from ref. ${ }^{74}$. The six-tenths rule is applied with total feed mass flowrate as attribute. Then, the bare module costs are estimated by considering the pieces of equipment as process vessels. The same procedure is used to cost the deaerator but using data associated with regular-scale deaerators.

Table 8 includes the calculated bare module costs of the modular equipment considered in the modular hydrogen unit. The process flow diagram nomenclature from Figure 36 is also adopted here. Details on individual cost calculation (material, design conditions, purchase cost, etc.) can be found in Appendix B. 
Table 8. Estimated modular bare module cost.

\begin{tabular}{|c|c|c|}
\hline Id. & scaling attribute & bare module cost \\
\hline E-101 & Area, $29.5 \mathrm{~m}^{2}$ & US\$ 160,366 \\
\hline$E-102$ & Area, $22.5 \mathrm{~m}^{2}$ & US\$162,922 \\
\hline$E-103$ & Area, $71.7 \mathrm{~m}^{2}$ & US\$ 198,737 \\
\hline E-104 & Area, $19.2 \mathrm{~m}^{2}$ & US\$163,205 \\
\hline E-105 & Area, $1.2 \mathrm{~m}^{2}$ & US\$ 517,480 \\
\hline$E-106$ & Area, $15.4 \mathrm{~m}^{2}$ & US\$163,965 \\
\hline F-101 & Duty, $1,050 \mathrm{~kW}$ & US\$ 682,216 \\
\hline $\mathrm{H}-101$ & Duty, 830 kW & US $\$ 851,823$ \\
\hline$V-101$ & Inlet flowrate, $285 \mathrm{~kg} / \mathrm{h}$ & US\$153,794 \\
\hline$V-102$ & Volume, $0.007 \mathrm{~m}^{3}$ & US\$11,854 \\
\hline$V-103$ & Inlet flowrate, $1,630 \mathrm{~kg} / \mathrm{h}$ & US\$14,064 \\
\hline$V-104$ & Inlet flowrate, $716 \mathrm{~kg} / \mathrm{h}$ & US $\$ 518,649$ \\
\hline$C_{B M, \bmod }^{1}$ & & US\$ 3,599,075 \\
\hline
\end{tabular}

In Table $8, C_{B M \text {,mod }}^{1}=$ US $\$ 3,599,075$ is the sum of all individual bare cost module costs and consists of the total bare module cost estimation for the first modular unit. The scale of this modular unit is associated with the nominal operation obtained in Subsection 7.3.2 and corresponds to a hydrogen production of about $0.32 \mathrm{Sm}^{3} / \mathrm{s}$. Note the differences in scale and cost: the modular unit has a hydrogen production and a bare module cost of $0.32 \mathrm{Sm}^{3} / \mathrm{s}$ and about US $\$ 3,600,000$, respectively, whereas the benchmark plant presents $29.5 \mathrm{Sm}^{3} / \mathrm{s}$ and US $\$ 78,300,000$, respectively. The obtained values of bare module cost will be employed for calculation of total modules costs, operating costs and scaled economic measures.

\subsubsection{Operating Cost Estimation}

For both SMR processes, total module costs remain a function of bare module costs and contingency. The total module cost for the modular hydrogen unit is also dependent on 
experience parameters and number of manufactured modules. As a result, the total module costs are expressed as $C_{T M, c o n}=C_{T M, c o n}\left(C_{B M, c o n}, a_{c o n}\right)$ and $C_{T M, \bmod }=$ $C_{T M, \text { mod }}\left(C_{B M, \text { mod }}^{1}, a_{\text {mod }}, \alpha, R_{\text {max }}, n\right)$. The values that allow for estimation of total module costs are explored in the following subsections.

The conventional plant has its cost of operating labor, $C_{O L, c o n}$, estimated considering that 22 operators are required for the operation of the hydrogen unit alone. The considered wage of an operator is the annual mean wage in chemical plants located in WV, in the range of the Marcellus Shale formation - a shale gas formation that would benefit from dynamic modular NG utilization. ${ }^{75}$ Table 9 shows the considered annual wage along with of prices adopted in the operating cost estimation such as raw materials, utilities, electricity and so on.

$C_{O L, m o d}$ is then estimated using Equation (75). The hydrogen productions of 29.5 $\mathrm{Sm}^{3} / \mathrm{s}$ for the conventional SMR and $0.32 \mathrm{Sm}^{3} / \mathrm{s}$ for the modular unit are converted to mass flow and assigned to as productions of chemical of interest $T_{H_{2}, \text { con }}$ and $T_{H_{2}, \text { mod }}$, respectively.

The cost of utilities and raw materials is comprised of the costs of air, cooling water, NG, and steam. The costs of air, cooling water and NG are determined by considering the respective flowrates in each process. The cost of steam, however, is estimated not only by accounting for the direct intake of chemicals, but also by calculating indirect consumptions such as power and circulating water treatment. To estimate the cost of steam, the costs of heating, treating and circulating water, air blowing, makeup boiler feedwater, and pumping boiler feedwater are considered. An electricity credit is applied to compensate for the presence of a steam turbine.

For the conventional SMR, the cost of heating can be neglected, since enough heat is supplied from the process streams and SMR furnace, which is fueled by PSA tail gas and NG - already considered. The costs of treating and circulating water, air blowing, and pumping are estimated using mass flowrates obtained from balances on the reference plant. Electricity credit is estimated using reported power production. 
For the modular unit, the cost of heating is also neglected because process heat is used to preheat boiler feedwater and the steam boiler is fueled solely by PSA tail gas. The costs of treating and circulating water and air blowing are estimated using the mass flowrates from the Aspen Plus simulation. Pumping cost and electricity credit are calculated using simulation values of pump net power and excess of heat from the steam boiler. For the latter, a turbine that presents $90 \%$ of thermal efficiency is considered.

The cost of waste treatment is simplified to the cost of wastewater treatment. For both processes, a unit for secondary wastewater treatment is considered available. Most of the wastewater is assumed to come from a blowdown or water purge, which is estimated to be about $10 \%$ of all circulated water.

Catalyst is considered for both cases, assuming yearly replacement. For the conventional SMR, reference values are employed. Whereas, for the modular hydrogen plant, the presence of catalysts is considered inside microchannel reactor, for combustion and various SMR reactions, and desulfurization unit. Both technologies leave catalyst replacement of PSA units out.

Table 9. Adopted market values.

\begin{tabular}{ll}
\hline item & value \\
\hline \hline Natural Gas & US $\$ 0.1119 / \mathrm{Sm}^{3}$ \\
Air & US $\$ 0.5 / 100 \mathrm{Sm}^{3}$ \\
Cooling water & US $\$ 15.7 / 1000 \mathrm{~kg}$ \\
Wastewater treatment & US $\$ 43 / 1000 \mathrm{~kg}$ \\
Circulating water treatment & US $\$ 0.156 / 1000 \mathrm{~kg}$ \\
Electricity & US $\$ 0.0674 / \mathrm{kWh}$ \\
High-purity water for process use & US $\$ 0.177 / 1000 \mathrm{~kg}$ \\
Microchannel reactor catalyst & US $\$ 0.2206 / \mathrm{pair}$ of microchannels \\
Desulfurization catalyst & US $\$ 355 / \mathrm{ft}^{3}$ \\
Average operator wage & US $\$ 67,350$ \\
\hline
\end{tabular}


As outcome of this step are values for the expressions $\operatorname{COM}_{\text {con }}\left(C_{B M, c o n}, a_{c o n}\right)$ and $C O M_{\text {mod }}\left(C_{B M, \text { mod }}^{1}, a_{\text {mod }}, \alpha, R_{\text {max }}, n\right)$. Assumptions regarding contingencies and experience curve are further studied in the following subsections for analysis of profitability.

\subsubsection{Base Case Scenario}

The experience curve is fit to literature values that indicate a maximum price decrease of around $42 \%$ at the $10^{\text {th }}$ produced modular unit. ${ }^{56}$ Using Equation (70), $R_{\max }$ is fixed at $42 \%$ and $\alpha$ is varied to reduce the mean square error between literature data points and model data points. Literature data is from ref. ${ }^{56}$ and refers to construction of modular nuclear reactors. Figure 9 shows both data points and the obtained model. The experience rate exponent that minimized the mean square error is $\alpha=0.24$, and along with $R_{\max }=42 \%$ defines a base case economy of learning for modular systems.

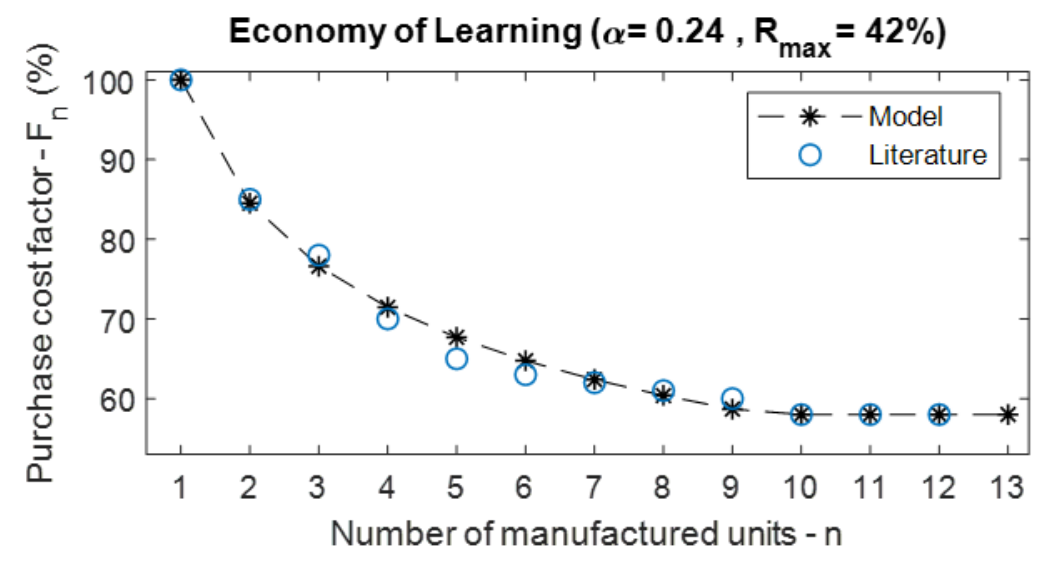

Figure 40. Experience curve: model fitting. Literature literature data points from ref. ${ }^{56}$.

Using the modeled experience curve and above results, total module and operating costs are calculated as well as scaled economic measures. For this scenario, the technology maturity is assumed for modular units. Therefore, values of $n \geq 10$ are considered, providing constant results for the $C_{T M, \bmod }$ and $\mathrm{COM}_{\bmod }$ calculations.

For both technologies, the following cost assumptions are considered:

- Standard of $18 \%$ for contingency and fees $\left(a_{c o n}=a_{m o d}=18 \%\right)$

- Plant lifetime of 25 years $\left(l f_{\text {con }}=l f_{\text {mod }}=25 \mathrm{yr}\right)$ 
- Interest rate of $6 \%$ per annum ( $i r_{c o n}=i r_{m o d}=6 \%$ per annum $)$

Then, comparison of scaled EAOC and its specific scaled terms, $C_{T M} \cdot P A$ and COM, is performed. Figure 41 contains the three economic measures. While the conventional SMR presents a scaled EAOC of about $1.3 \mathrm{US} \$ / \mathrm{kg}_{\mathrm{H}_{2}}$, scaled annuitized capital cost of $0.1 \mathrm{US} \$ / \mathrm{kg}_{\mathrm{H}_{2}}$ and scaled operating cost of $1.2 \mathrm{US} \$ / \mathrm{kg}_{\mathrm{H}_{2}}$, the modular unit has values of $1.9 \mathrm{US} \$ / \mathrm{kg}_{\mathrm{H}_{2}}, 0.24 \mathrm{US} \$ / \mathrm{kg}_{\mathrm{H}_{2}}$ and $1.7 \mathrm{US} \$ / \mathrm{kg}_{\mathrm{H}_{2}}$, respectively. This result indicates a trend of the modular unit to present a higher overall hydrogen cost due to both capital and operating costs.

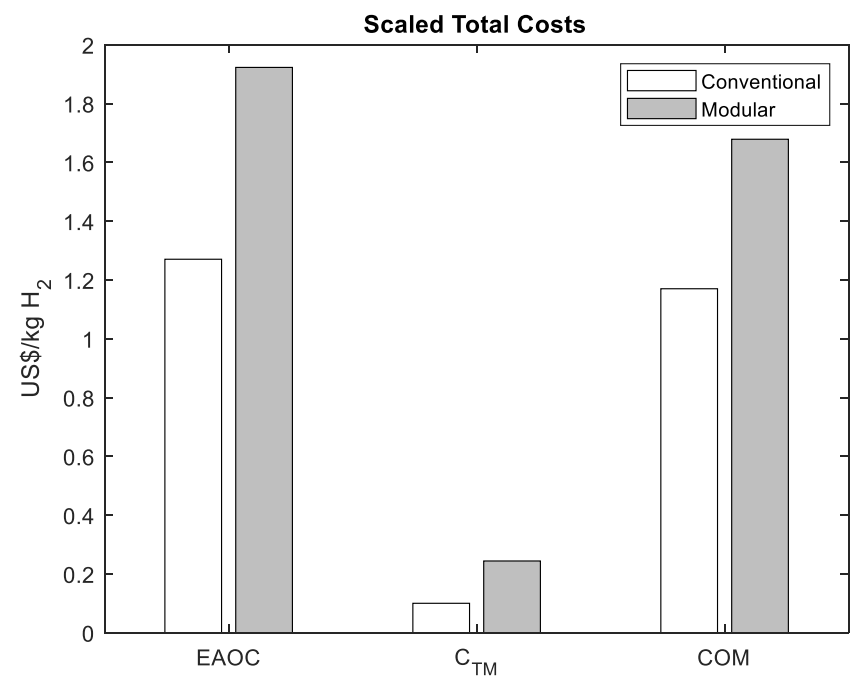

Figure 41. Comparison of scaled costs: EAOC, total capital cost and operating cost. 


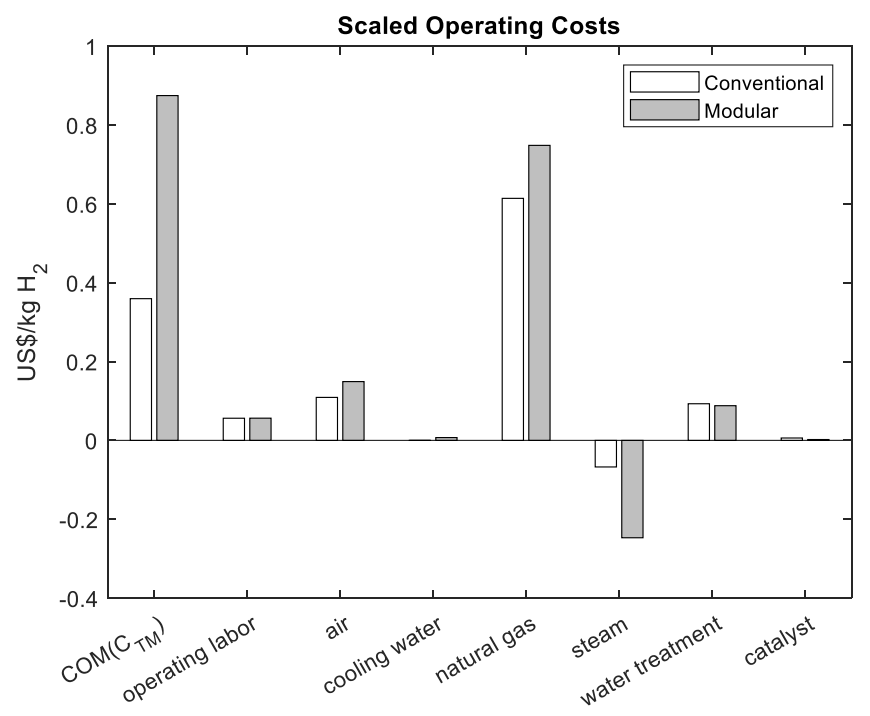

Figure 42. Detailed comparison of scaled operating costs.

As modular intensified technologies are expected to have better efficiencies, the scaled operating costs are further detailed using the following terms: (i) depreciation, maintenance and repairs, operating supplies and other operating costs that depend on total capital cost, which is represented by $\operatorname{COM}\left(C_{T M}\right)=0.280 \cdot C_{T M}$; (ii) the operating labor, represented by $2.73 \cdot C_{O L}$; and (iii) specific terms associated with raw materials, utilities, waste treatment and catalyst replacement, which are represented by $1.23 \cdot C_{i}$, where $C_{i}$ refers to each of the specific costs. These terms are scaled and illustrated in Figure 42.

In Figure 42, the main differences in operating costs are due to the terms associated with capital cost, $\operatorname{COM}\left(C_{T M}\right)$. Identical scaled costs of operating labor are a direct result of assumptions adopted using Equation (75). A slightly higher consumption of air is identified in the modular unit, mainly because of the occurrence of combustion in two unit operations consisting of a microchannel reactor and steam boiler as opposed to just one in the conventional process, the reforming furnace. The increased NG intake in the modular unit is a consequence of fueling the combustion microchannel with just methane. A lower steam cost in indicated for the modular unit, which is caused by a relatively higher production of electricity. The magnitude of the costs in Figure 42 indicate that terms associated with capital cost and cost of NG are the most significant factors for increasing the cost of hydrogen for the modular system. 
Because capital cost is relevant in this comparison, experience parameters, modular contingency and fees, interest rate, and lifetime should be further investigated. Differences in cost of air, cooling water, water treatment and catalyst are slim and low in magnitude when compared to NG costs. As variations in price of NG are prone to happen in certain scenarios - particularly where natural is currently flared, NG price is also considered for further investigation.

\subsubsection{Sensitivity Analysis}

Here, potential improvements in the scaled EAOC of the modular hydrogen unit are analyzed. Investigated variables are changed within an expected range, while other parameters are all held at the values described in Subsection 7.3.3.3. The sensitivity of the scaled modular EAOC is studied with respect to purchase cost reduction due to economy of learning $\left(R_{n}\right)$, modular project contingency and fees $\left(a_{\text {mod }}\right)$, modular unit lifetime $\left(l f_{\text {mod }}\right)$, interest rate of modular project $\left(i r_{m o d}\right)$ and price of NG where modular unit is operated $\left(P_{N G, m o d}\right)$.

For the economy of learning, variation in the experience rate exponent, $\alpha$, can be only be accurately investigated in conjunction with the analyzed number of manufactured modular units and maximum reduction in cost, $R_{\max }$. The simplification to purchase cost reduction $R_{n}$ is adopted as alternative to the study of the 3 experience parameters. Values of $R_{n}$ are the reduction in purchase cost observed for the entire experience curve, representing either cost reductions during the learning phase or $R_{\max }$ at technology maturity. For the sensitivity, $R_{n}$ is tested within the range of $20-90 \%$.

Regarding other variables, modular unit lifetime is analyzed within a range in which the lower bound of 5 years corresponds to a relatively low lifetime for a chemical plant, and the upper bound of 60 years corresponds to longer lifetimes, based in extreme cases such as nuclear modular reactors. The contingency and fees range is based on the expectation of contingency between $15 \%$ and $55 \%$ for technologies that follow an experience curve. ${ }^{56}$ Supposing changes in fees from fiscal incentives, the minimum value for modular contingency and fees, $a_{\text {mod }}$, is at $15 \%$ and maximum, $58 \%$. 
Similarly, the interest rate is varied between 1 and $10 \%$ p.a., corresponding to situations of low and high interests. NG price is analyzed for situations in which it is bought close to its market value and situations in which price declines due to stranded locations. For the first situation, the NG price upper bound is rounded up to US $\$ 0.012 / \mathrm{Sm}^{3}$, based on the price depicted in Table 9. For the lower bound, free NG is considered. For none of the investigated parameters, financial stimulus - which would turn certain costs into credits, is considered.

All system parameters are held at the values described in Subsection 7.3.3.3. Then $R_{n}, a_{\text {mod }}, l f_{\text {mod }}, i r_{\text {mod }}$, and $P_{N G, \text { mod }}$ are changed individually within the expected ranges described above. The changes in scaled EAOC are monitored as each variable is changed. As a result, a sensitivity curve is obtained for each parameter: $R_{n}, a_{\text {mod }}, l f_{\text {mod }}$, $P_{N G, \text { mod }}$ and $i r_{\text {mod }}$ vs. scaled EAOC. Figure 43 shows each of these curves. The base case values from Subsection 7.3.3.3 are represented as data points in the respective sensitivity curve for each analyzed variable. The scaled EAOC values corresponding to both modular and conventional technologies are included as horizontal lines.

The price of NG and reduction due to economy of learning display higher impacts on the $E A O C$. Price of NG alone presents potential to bring the modular unit to break-even scenarios when significantly decreased. The economy of learning, however, requires reductions of above $75 \%$ in purchase cost to do the same, which may not be realistic.

Contingency and fees come next in order of relevance. Higher values of contingency and fees may drift the modular technology away from competitive scenarios, if not compensated by other variables. Interest rate changes from the base case are insufficient to cause significant influence on final cost. Modular unit lifetime only plays a relevant role to around 18 years.

The assumption that a modular unit has a lifetime of at least 18 years is thus adopted. Therefore, both lifetime and interest rate are ruled out in further analysis. The combined effect of cost reduction due to the economy of learning, price of NG and contingency and fees is studied next. The following step analyzes cases in which these three variables are changes simultaneously for competitiveness of the modular process. 


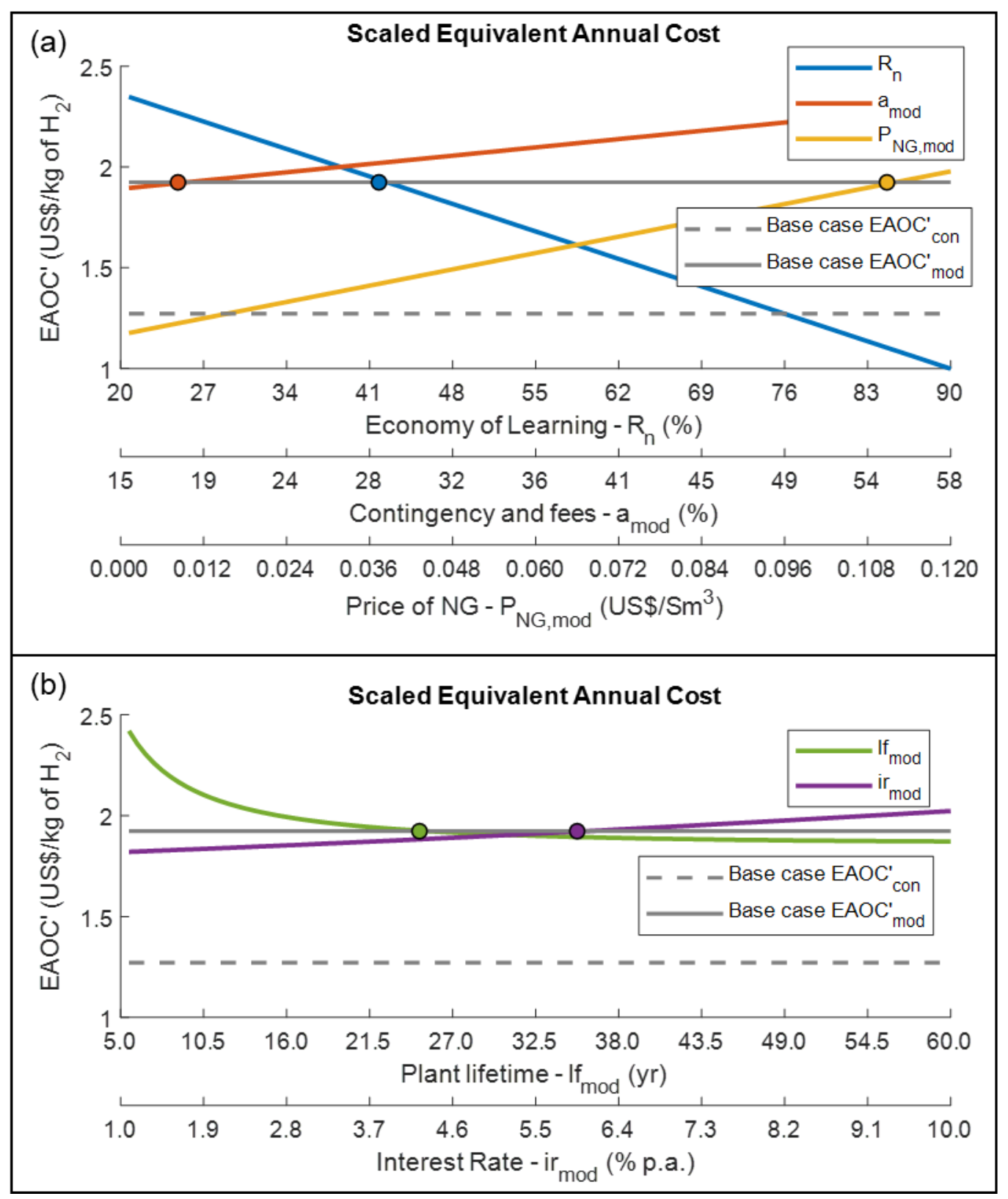

Figure 43. Sensitivity analysis of scaled EAOC of modular unit: (a) $R_{n}, a_{\text {mod }}$ and $P_{N G, \bmod }$ and (b) $l f_{\text {mod }}$ and $i r_{\text {mod }}$. 


\subsubsection{Profitability Scenarios}

\section{Case (i): Profitability of a modular hydrogen unit}

The first profitability case is associated with individual modular units. NG price, contingency and fees and reduction due to economy of learning have their combined effect mapped to profitability scenarios. The variables are changed within the same ranges as in Subsection 7.3.3.4.

The aimed scenarios consist of achievement of break-even scaled EAOC and more aggressive reductions in price in comparison with the conventional SMR. Therefore, the variables $P_{N G, \text { mod }}, a_{\text {mod }}$ and $R_{n}$ are mapped to $E A O C_{m o d}^{\prime}$. The studied profitability scenarios correspond to the following:

- Break-even cost of hydrogen: $E A O C_{m o d}^{\prime}=E A O C_{c o n}^{\prime}$

- Reduction of $25 \%$ in cost of hydrogen for the modular case: $E A O C_{m o d}^{\prime}=75 \%$. $E A O C_{\text {con }}^{\prime}$

- Reduction of $50 \%$ in cost of hydrogen for the modular case: $E A O C_{m o d}^{\prime}=50 \%$. $E A O C_{c o n}^{\prime}$

- Reduction of $75 \%$ in cost of hydrogen for the modular case: $E A O C_{\text {mod }}^{\prime}=25 \%$. $E A O C_{\text {con }}^{\prime}$

Combinations of $P_{N G, m o d}, a_{m o d}$ and $R$ that map to each scenario consist of a set in $\mathbb{R}^{3}$ that maps to a value in $\mathbb{R}$, corresponding to the aimed profitability scenario. To find a representation of those sets, Equation (78) is further developed for the modular unit. By substituting $C_{B M, \text { mod }}^{n}, C_{T M, \text { mod }}, C O M_{\text {mod }}$ and $E A O C_{m o d}$, by Equations (72), (73), (74) and (76), respectively, setting the cost of NG expression apart from the cost of other utilities/raw materials, Equation (82) is obtained.

$$
E A O C_{m o d}^{\prime}=\frac{\left(1+a_{m o d}\right) \cdot\left(1-R_{n}\right) \cdot C_{B M, m o d}^{1} \cdot(P A+0.280)+2.73 \cdot C_{O L, m o d}+1.23 \cdot\left(P_{N G, m o d} \cdot V_{N G, m o d}+X\right)}{P_{H_{2}, m o d} \cdot O P}
$$

where $X=\tilde{C}_{U R M}+C_{W T, \text { mod }}+C_{C A T \text {,mod }}$ corresponds to the sum of $\tilde{C}_{U R M}$, which is the cost of utilities and raw materials, excluding the cost of NG; $C_{W T, m o d}$ is the cost of waste treatment; and $C_{C A T, \text { mod }}$ is the catalyst replacement cost. 
The modular unit is analyzed for a nominal operation and a certain design. Therefore, bare module cost and variables associated with operating cost are fixed. Supposing cases for which $a_{m o d}$ is fixed at particular values, Equation (82) can be interpreted as a linear equation of the following type:

$$
E A O C_{\text {mod }}^{\prime}=K_{1} \cdot R_{n}+K_{2} \cdot P_{N G, \bmod }+K_{3}
$$

where $K_{1}, K_{2}$ and $K_{3}$ are constants that depend on the adopted value of contingency and fees.

Contingency and fees are divided into extreme cases, i.e., $a_{\text {mod }} \in$ $\{15 \%, 18 \%, 58 \%\}$. Then, for each case, 4 points of the complete economic model are simulated and values of $K_{1}, K_{2}$ and $K_{3}$ are found. The range of $P_{N G, m o d}$ is divided into 100 points, $E A O C_{m o d}^{\prime}$ is set to a target value, and Equation (83) is solved for $R_{n}$. As a result, if achievable, combinations of $R_{n}$ and $P_{N G, m o d}$ that result in the target $E A O C_{m o d}^{\prime}$, given $a_{m o d}$, are obtained.

This procedure is repeated for each $a_{m o d}$ and each profitability scenario. The results are plotted in Figure 44, in which each color represents a profitability scenario. 


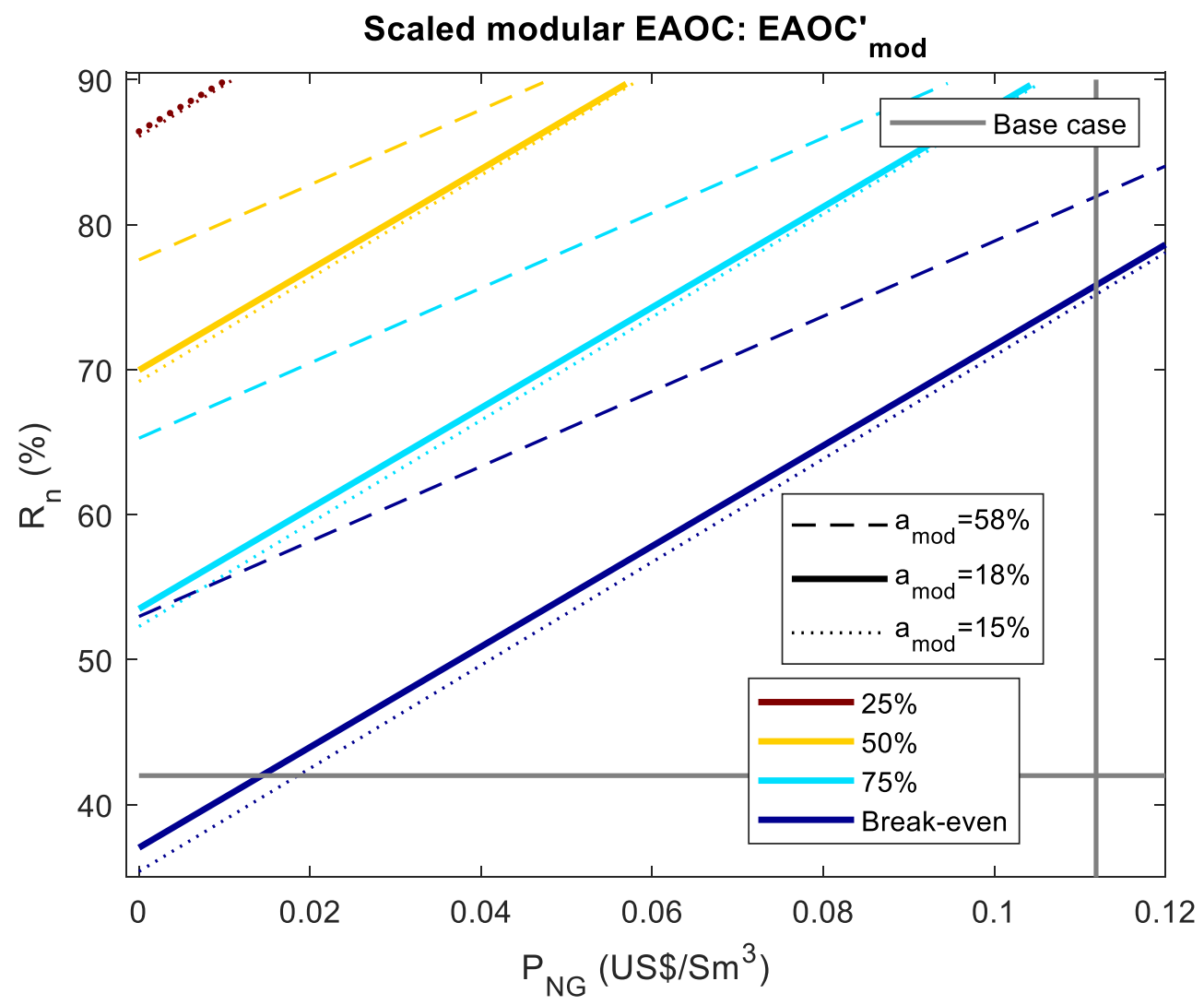

Figure 44. Achievability of profitability scenarios. Faded gray lines represent values from the base case.

Figure 44 indicates that NG price is the most decisive factor for economic feasibility of the modular hydrogen unit. The considered NG market value of US $\$ 0.1119 / \mathrm{Sm}^{3}$ is indicated by a vertical line related to the base case. For NG at market value, a purchase cost reduction due economy of learning between around $77 \%$ and $83 \%$ is required, depending on the contingency and fees. Even with low fees and high reliability on cost data, the required reductions seem unrealistic.

The break-even and $75 \%$ of $E A O C_{c o n}^{\prime}$ scenarios display potential when NG price decreases below $0.02 \mathrm{US} \$ / \mathrm{m}^{3}$. In this case, required reductions in purchase cost due to economy of learning are lower and approach the reference of $42 \%$, implying that the modular unit can become competitive and slightly more profitable. More aggressive scenarios are likely to be unattainable because they require not only lower NG prices, but also higher reductions in purchase cost. The most aggressive scenario is of $25 \%$ of 
$E A O C_{c o n}^{\prime}$ and would also require no fees and standard values of contingency, which is only observed in well-known technologies.

There is a tradeoff between contingency and fees and required $R_{n}$, suggesting that increase in reliability on flowsheet completion and modular cost data may result in more profitability. Naturally, first-of-a-kind modular designs should be associated with the upper cost situation, where $a_{m o d}=58 \%$. Similarly, modular designs at maturity would be associated with lower cost situations, where $a_{\text {mod }}$ approaches $15 \%$ without fees and $18 \%$ for standard fees of $3 \%$.

This study indicates that the developed modular hydrogen unit competes with the conventional SMR only when NG prices fall below $0.02 \mathrm{US} \$ / \mathrm{Sm}^{3}$. Placement of modular hydrogen units where NG is abundant (remote locations and/or when flared) would describe the situation in which NG price is low for the modular technology but is at market value for the regular plant - installed where infrastructure is more robust and NG demand is higher. The economy of learning suggests that the modular technology has potential to be more profitable as it approaches maturity. The effects of both reduction in price due to accrued knowledge and reliability in cost data and flowsheet play in favor of the modular technology.

\section{Case (ii): Profitability of a modular hydrogen plant}

The bulk or massive purchase of modular units is studied to link the experience curve phenomena and modular deployment. The modular hydrogen units are placed in parallel to produce the same amount of hydrogen as the considered conventional SMR plant. Bulk purchase is assumed, and the units should start-up at the same time at a standard nominal operation. The cost model must be adapted to accommodate purchase costs that change with the experience curve.

The capital and operating costs are adapted to include differences between each purchased modular unit. Equation (73) is modified to represent the cost of the entire modular plant rather than the total cost of a modular unit. Equation (84) contains the total 
module cost expression for the modular plant. It is assumed that all units present the same value of contingency and fees.

$$
C_{T M, M P}=\left(1+a_{m o d}\right) \cdot C_{B M, \text { mod }}^{1} \sum_{i=1}^{n} F_{i}
$$

where $C_{T M, M P}$ is the total module cost of the modular plant, $a_{m o d}$ are the contingency and fees, $C_{B M, \text { mod }}^{1}$ is the bare module cost of the first hydrogen unit, $F_{i}$ is the multiplier for cost reduction for each ith modular unit, and $n$ is the total number of purchased modular units.

Equation (74) is modified for calculation of the operating costs. Assuming all units operate at the same nominal point, Equation (85) shows the operating cost of the entire modular plant.

$$
\operatorname{COM}_{M P}=0.280 \cdot C_{T M, M P}+n \cdot\left[2.73 \cdot C_{O L}+1.23 \cdot\left(C_{U R M}+C_{W T}+C_{C A T}\right)\right]
$$

in which $C O M_{M P}$ is the operating cost of the modular plant, $C_{T M, M P}$ is the total module cost, $n$ is the total number of purchased modular units, and the other variables follow the notation of Equation (74) and refer to the operation of an individual unit.

Equations (84) and (85) are then used for estimation of capital and operating costs. Then, Equations (76), (77) and (78) are applied for calculation of the scaled EAOC for the modular hydrogen plant.

The number of purchased units is calculated considering the productions of hydrogen of a modular unit and conventional SMR. As a result, a total of 92 modular units composes the modular hydrogen plant. This modular plant achieves a similar production of hydrogen, which consists of $29.55 \mathrm{Sm}^{3} / \mathrm{s}$.

The scaled EAOC for the modular plant is contrasted with the scaled EAOC of individual modular units. Assuming an experience rate exponent of $\alpha=0.15$, maturity at $R_{\max }=40 \%$ and free NG, the values of scaled EAOC are calculated for the first 100 modular units, and, thus, for modular plants that contain a total number of units that range from 1 to 100. Figure 45 explores the differences between the two scaled EAOC curves. The curve associated with the modular units represents the scaled EAOC for individual units. For example, the $22^{\text {nd }}$ and $30^{\text {th }}$ produced units have scaled costs of 1.27 and 1.21 
US $\$ / \mathrm{kg} \mathrm{H}_{2}$, respectively. The curve associated with the scaled $E A O C$ for the modular plant represents the cumulative cost of the assembled plant with a given number of units. For example, a modular plant that is bulk purchased and contains the first 22 modular units costs about $1.46 \mathrm{US} \$ / \mathrm{kg} \mathrm{H}_{2}$. Similarly, a modular plant that assembles the first 30 modular units has a total of $1.4 \mathrm{US} \$ / \mathrm{kg} \mathrm{H}$.

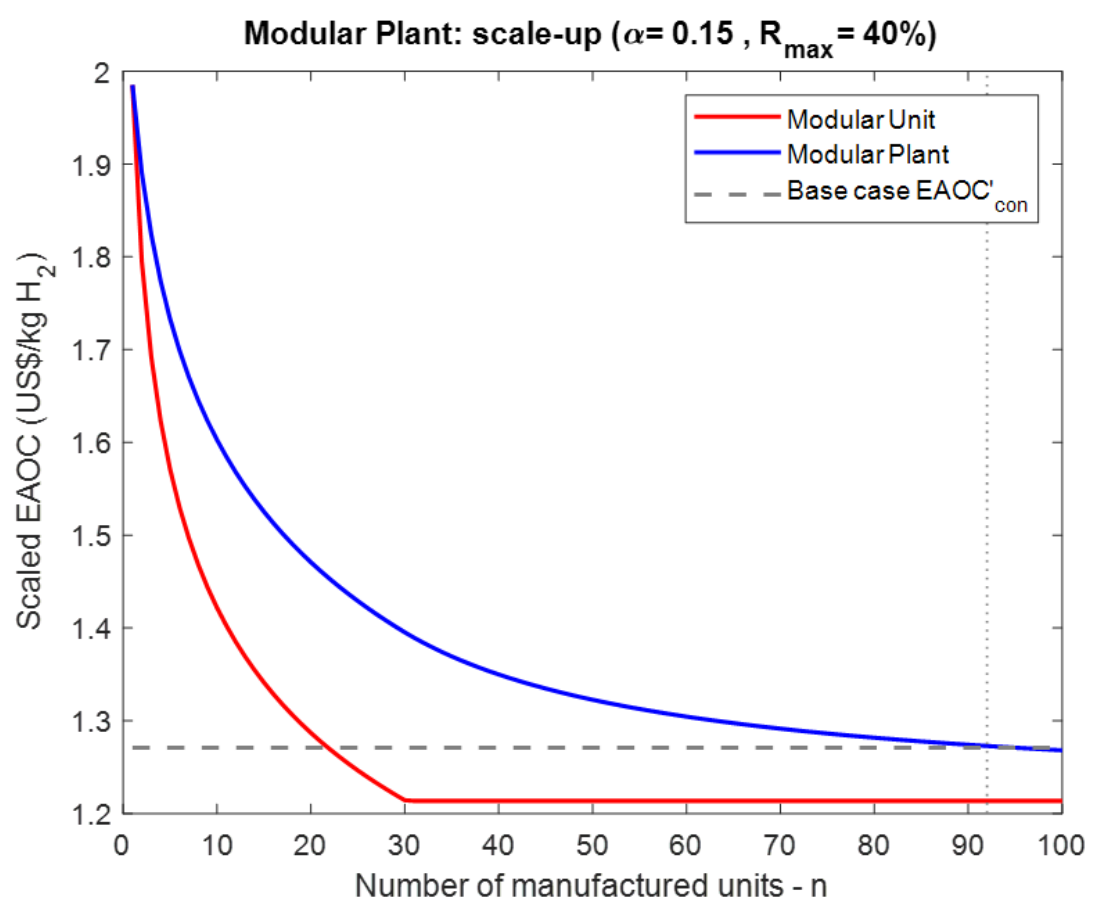

Figure 45. Differences between scaled EAOC of a unit and of the plant (cumulative) $P_{N G}=0 \mathrm{US} \$ / \mathrm{Sm}^{3}$. Dotted faded lines correspond to the number of units 92 , required to achieve regular hydrogen production.

The trend from Figure 45 shows that, in general, for the adopted parameters, the modular units achieve break-even faster, at around the $21^{\text {st }}$ produced modular unit. For the modular plant, however, break-even only occurs when the modular plant has around 90 modules. This happens because the price reduction from the second and subsequent units compensates for the higher purchase costs of the beginning of the learning process.

Values of $\alpha$ and $R_{\max }$ that provide break-even cost for the modular plant are determined. Two extreme scenarios of NG price are considered: free NG and market value. The scaled EAOC function is written as a function of NG price, $\alpha$ and $R_{\max } .100$ points are obtained by discretizing the entire range of $\alpha$. Finally, for each NG price 
scenario, each point is solved to obtain the value of $R_{\max }$ that provides break-even, or $E A O C_{c o n}^{\prime}$, given $\alpha$.

The MATLAB routine Isqnonlin is employed to find each value $R_{n}$. It corresponds to nonlinear least-squares optimization problem. The minimized objective function is set as the difference $E A O C_{M P}^{\prime}-E A O C_{c o n}^{\prime}$, in which $E A O C_{M P}^{\prime}$ is the scaled $E A O C$ of the modular plant. $R_{\max }$ is limited by upper and lower bounds corresponding to the range of $20 \%$ $90 \%$. The range of $\alpha$ of $0.1-0.9$ is selected to model a reasonable experience curve without drastic decreases in cost.

The routine is solved with the default "trust-region-reflective" algorithm for each point, providing values of $R_{\max }$ that bring $E A O C_{M P}^{\prime}$ the closest to $E A O C_{c o n}^{\prime}$. The residual values are only used to select $\alpha$ and $R_{\max }$ points that actually provide $E A O C_{M P}^{\prime}=$ $E A O C_{c o n}^{\prime}$. Points with residuals greater than $1 \cdot 10^{-6}$ are discarded.

Figure 46 shows the mapping of leaning parameters for the NG price scenarios. When NG is free, the experience curve can behave in a wider range of $\alpha$ and $R_{\max }$ values. Whereas when NG is bought at market value, more aggressive learning phenomena should be followed to provide break-even.

The required regions of experience parameters are bounded by minimum requirement of experience rate exponent and maximum reduction in price for both price scenarios. The boundaries correspond to curves that suggest tradeoff between $R_{\max }$ and $\alpha$. Compromise between the two parameters appear towards the left bottom corner, and beyond this region, $\alpha$ and $R_{\max }$ grow rapidly. Therefore, balanced and desired learning phenomena are represented by these portions of the limiting curves. Note that breakeven is only achieved for combinations of $\alpha$ and $R_{\max }$ that fall on the boundary of the illustrated regions. Any point inside the depicted regions provides situations that are better than the break-even.

For free NG, less aggressive conditions are related to minimum requirements. An experience rate exponent of around 0.15 and a maximum reduction in price of about $38 \%$ are sufficient to achieve break-even. For NG at market price, minimum values of about 0.45 and $80 \%$, respectively, are obtained, which corresponds to a much more aggressive 
learning behavior. If NG price falls in between the analyzed scenarios, the required learning behavior is expected to be bounded by a curve that is also located between the ones associated with each scenario.

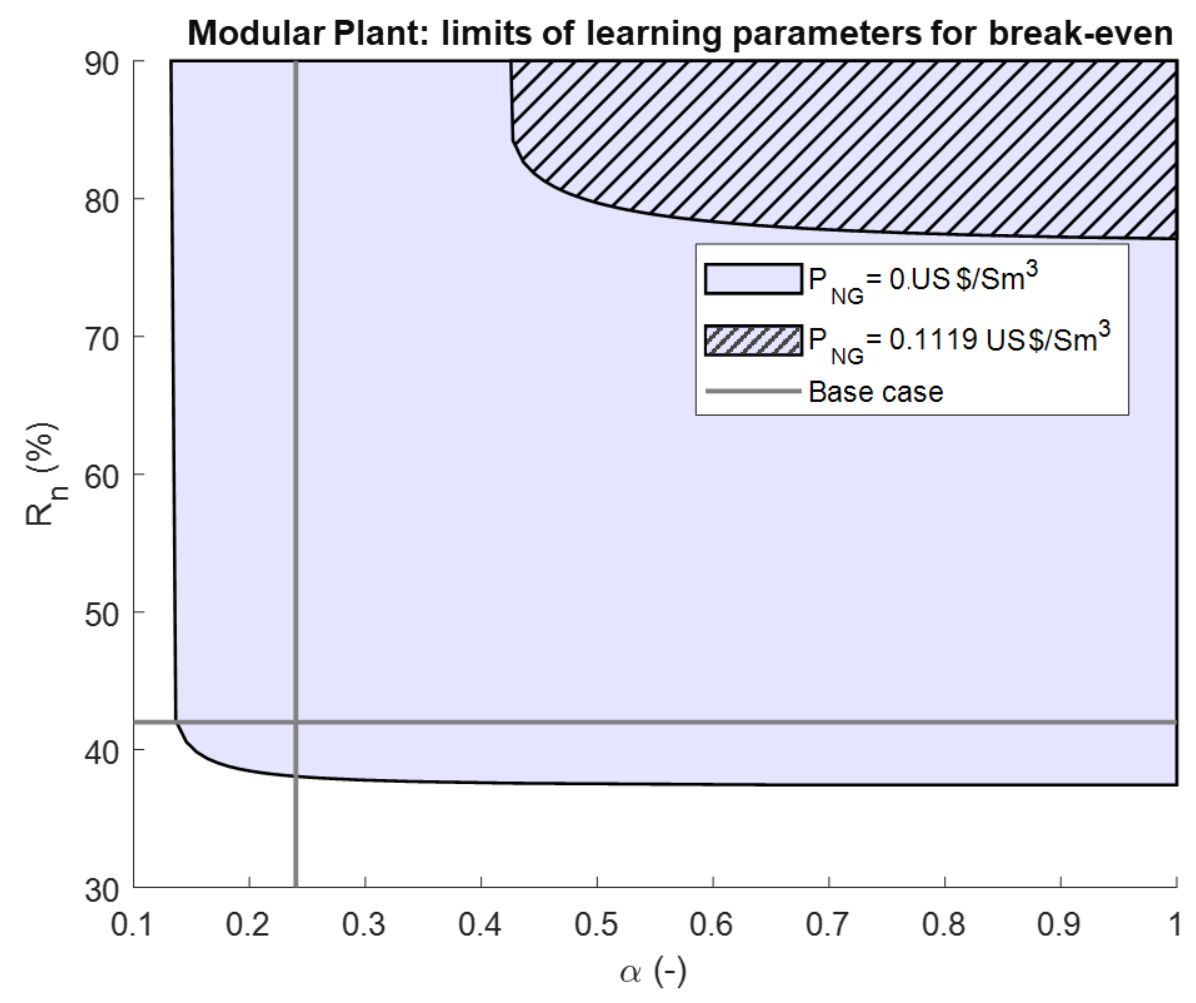

Figure 46. Mapping of leaning parameters for NG price scenarios. Base case lines represent values of $\alpha=0.24$ and $R_{\max }=42 \%$, modeled after literature.

Feasible values for both NG price scenarios are chosen to illustrate the outcome of this analysis. Experience rate exponent and maximum price reductions of 0.15 and $40 \%$, respectively, are both greater than the minimum values of 0.15 and $38 \%$ for breakeven when NG is free. Figure 45 depicts this situation, and as previously stated, modular plants constituted by 90 modular units or more present $E A O C_{M P}^{\prime}$ that at least break-even with $E A O C_{\text {con }}^{\prime}$.

An experience rate exponent of 0.5 , and a price reduction of $80 \%$ are values that fit the requirement for break-even cost when NG is at market value. Figure 47 shows the different scaled EAOC curves for individual modular units and modular plant in this situation. In comparison with the trend of Figure 45, a steeper and faster decrease in price 
is required. Technology maturity is achieved at around the $30^{\text {th }}$ manufactured modular unit for the previous case due to a slow experience rate exponent. Here, maturity happens at around 25 modular units, because of a more aggressive experience rate exponent is needed to achieve doble of the reduction in price.

In general, the bulk purchase of modular units can be used to offset higher purchase costs until technology reaches maturity. A modular plant can be competitively assembled to produce the same amounts of hydrogen as the conventional SMR process when NG prices are closer to zero. When NG is at typical market values, high reductions in price must be present for a longer time, which might not be feasible in practice.

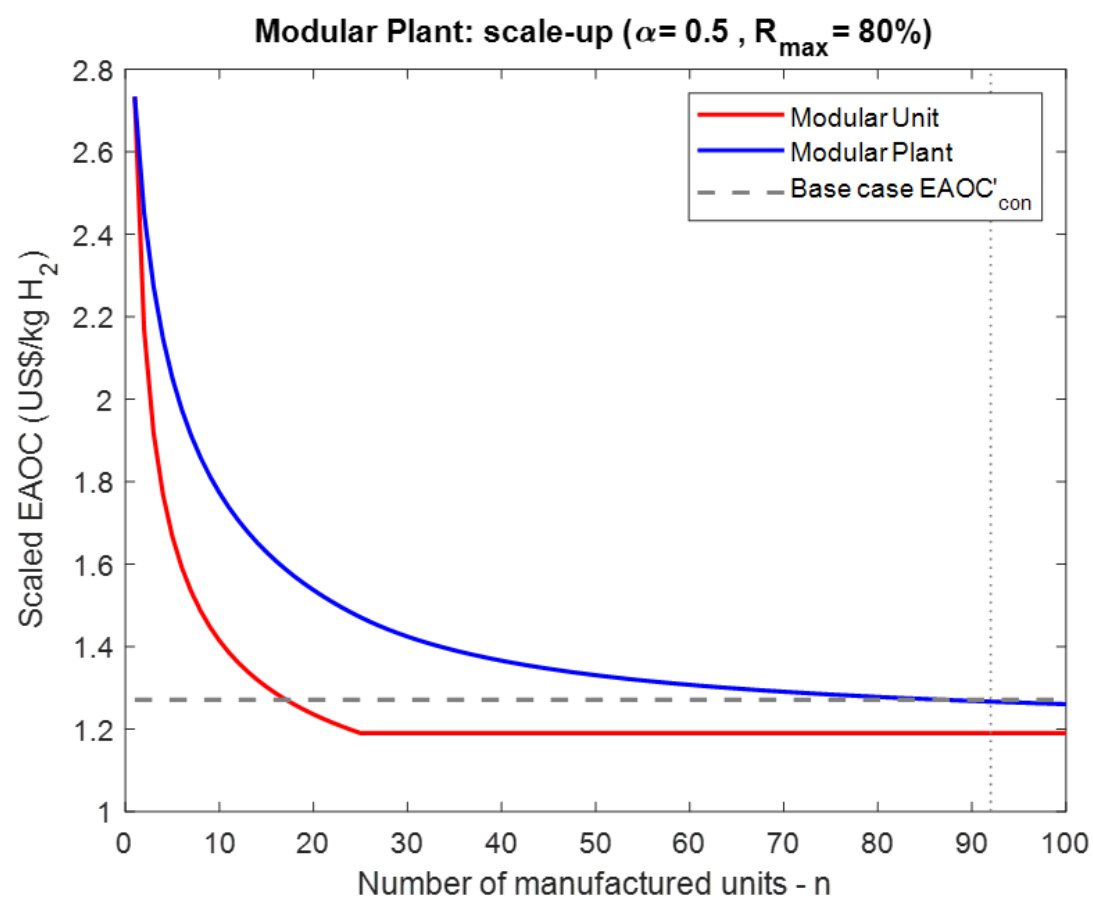

Figure 47. Differences between scaled EAOC of a unit and of the plant (cumulative) $P_{N G}=0.1119 \mathrm{US} \$ / \mathrm{Sm}^{3}$. Dotted faded lines correspond to the number of units of 92 , required to achieve regular hydrogen production. 


\subsubsection{Flexibility Analysis}

The nominal operation and equipment design were previously determined considering a certain amount of produced heat, process streams specifications, and so on. The developed modular process presents a strong heat integration scheme that relies on these nominal specifications. Changes to the modular unit operation can take the system to infeasible situations such as: (i) a temperature cross inside a heat exchanger, (ii) not enough PSA tail gas to fuel the steam boiler and produce steam, and (iii) water cannot be knocked out at the flash drum because the stream temperature is too high. Here, operability analysis is used to make sure the developed modular process is flexible enough to withstand changes in operation caused by disturbances in the NG feed composition.

The same definitions of the inputs and outputs from Subsection 7.3.2 are adopted. The EDS and DOS are then redefined for calculation of $\mathrm{r}-\mathrm{O}$. A range of methane concentration in the inlet $\mathrm{NG}$ of $90 \%$ to $100 \%$, defining the EDS as $E D S=$ $\left\{d_{1} \in \mathbb{R} \mid 90 \leq d_{1} \leq 100\right\}$. The methane content in NG widely varies and is dependent on particularities of the corresponding reservoir and recovery techniques. The chosen range represents rounded values that are reasonably close to $\mathrm{NG}$ at pipeline quality - methane concentrations higher than $99.3 \%$. The DOS is modified to DOS $=y^{N}$, in which $y^{N}=$ $\left(y_{1}^{N}, y_{2}^{N}, y_{3}^{N}\right)=(92.57,0.32,96.56)$ is the nominal operation described in Subsection 7.3.2.

All the blocks in the Aspen Plus model that were in design mode are converted to simulation mode using equipment information from Table 8 . This change causes the simulator to stop redesigning the equipment and simulate actual system behavior. The modular unit is designed for nominal operation. Therefore, the system behaves in the same way when operated at nominal point in simulation mode. However, if disturbances and MVs are different than $d^{N}$ and $u_{o p}^{N}$, respectively, an operation different than nominal is evaluated for equipment at a fixed design.

The set $A I S_{o p}^{\prime}=A I S_{o p} \times E D S=\left\{\left(u_{o p}, d_{1}\right) \mid u_{o p} \in A I S_{o p}\right.$ and $\left.d_{1} \in E D S\right\}$ is initially pre-screened to provide insights about infeasible situations. The set $A I S_{o p}^{\prime}$ is evenly divided into $4^{2}=16$ extreme points corresponding to combinations of lower and upper 
bounds of $A I S_{o p}$ and $E D S$. Each of these points is simulated and analyzed in Aspen Plus. As a result, violations related to heat exchangers E-104 and E-106 are observed due to temperature cross. The other unit operations are simulated without warning or errors. Process specifications are respected for all situations, except for a high temperature in the stream directed to the flash drum V-102. The amount of heat produced from tail gas is always enough for the required steam production at the steam boiler.

The prescreening results motivate an analysis of heat exchangers E-104 and E106. The AIS is augmented to include two design parameters: $u_{\text {des }, 1} \stackrel{\text { def }}{=}$ Area of $\mathrm{E}-$ $104\left(\mathrm{~m}^{2}\right)$ and $u_{d e s, 2} \stackrel{\text { def }}{=}$ Area of $\mathrm{E}-106\left(\mathrm{~m}^{2}\right)$. In order to define the ranges of the AIS des, the blocks E-104 and E-106 are turned into design mode. Then, simulations are run using the 16 extreme points of $A O S_{o p}^{\prime}$, providing 16 design combinations $\left(u_{d e s, 1}, u_{d e s, 2}\right)$. The ranges of obtained values define the lower and upper bounds of AIS des as shown in Table 10 below.

Table 10. Design input variables and available ranges.

\begin{tabular}{ll}
\hline design input variable & available range \\
\hline \hline Area of $\mathrm{E}-104\left(\mathrm{~m}^{2}\right)$ & $16-23$ \\
Area of $\mathrm{E}-106\left(\mathrm{~m}^{2}\right)$ & $11-20$ \\
\hline
\end{tabular}

The AIS comprises both design and operational variables within available ranges. The EDS contains the expected ranges of perturbations in methane inlet concentration. The DOS is assumed as the nominal point. These operability sets are used in the $2^{\text {nd }}$ layer of the multilayer framework, which was introduced in Subsection 5.2.2. The goal is to find designs that maximize the value of $\mathrm{r}-\mathrm{Ol}$, and therefore, provide more flexibility under the presence of disturbances.

A MATLAB script for connection to the Aspen Plus simulation is created to change these inputs and disturbance and collect outputs, and the log with status of the simulations. Simulations that present errors or warning are considered unachievable. The corresponding data points are removed from the input-output mapping. The Process Operability App is used to automatically calculate the values of $r$-OI as a function of each 
design combination, i.e., $r-O I\left(A I S_{d e s}\right)$. The even discretization of AIS des, AISop' and DOS' are defined by divisions of 5,3 and 5 , respectively. As a result, $5^{m_{\text {des }}}=5^{2}=25$ designs are analyzed and, for each design, $3^{m_{o p}+q}=3^{4}=81$ AIS'op $\times$ AOS' points are used to obtain one multimodel representation. Note that these calculations of $\mathrm{r}$-OI employ concepts presented in Subsections 3.1.2 and 4.2.

Figure 48 depicts the obtained designs ranked according to $r$-OI. Smaller heat exchanging areas provided $r$-Ol values of $100 \%$, indicating that the nominal output point represented by $D O S=y^{N}$ can be achieved for all disturbance scenarios. Bigger heat exchangers present lower $\mathrm{r}$-OI values., indicating that at certain disturbance scenarios, the nominal setpoint is unachievable, given the available MVs.

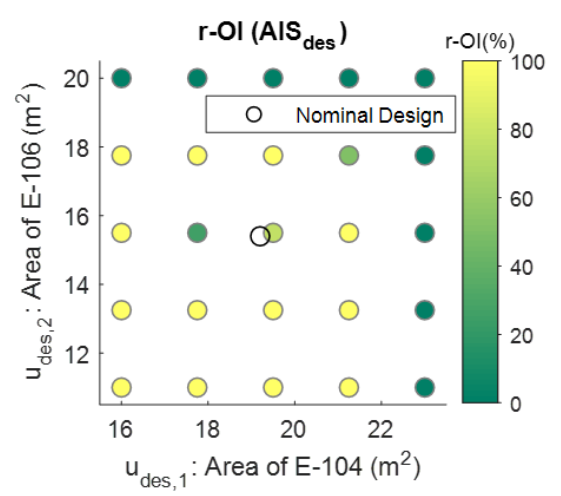

Figure 48. Operability analysis of AIS des using r-OI.

The behavior of $r$-Ol in terms of achieving the set $\operatorname{DOS}^{\prime}=\left\{\left(y^{N}, d\right) \in \mathbb{R}^{4} \mid d \in E D S\right\}$ is demonstrated for two designs: $u_{\text {des }}=(16,11)$ and $u_{\text {des }}=(23,20)$ with $r$-Ol values of $100 \%$ and $0 \%$ respectively. The input and output spaces are appended with the disturbance dimension in the multimodel representation. Therefore, the AIS 'op-AOS' mapping is of type $\mathbb{R}^{4} \rightarrow \mathbb{R}^{4}$. Projections of AIS' op on dimensions $\left(u_{o p, 1}, d_{1}\right),\left(u_{o p, 2}, d_{1}\right)$ and $\left(u_{o p, 3}, d_{1}\right)$ are used to illustrate input polytopes, whereas projection of AOS' and DOS' on dimensions $\left(y_{1}, d_{1}\right),\left(y_{2}, d_{1}\right)$ and $\left(y_{3}, d_{1}\right)$ are used to illustrate the corresponding output polytopes.

Figures 49 and 50 show the projections for the design $u_{\text {des }}=(16,11)$. In Figure 49 , projected polytopes in red represent how each MV is employed according to the specific values of methane concentration. Figure 50 contains corresponding output 
polytopes and their projections. As the DOS' represents achievability of $y^{N}$ at all disturbance scenarios, its projections are constant values in each output dimension, which is illustrated by the straight vertical line. This result indicates that the design $u_{\text {des }}=$ $(16,11)$ can be operated within the given MVs ranges to maintain the system's CVs at their nominal point at all disturbance scenarios.
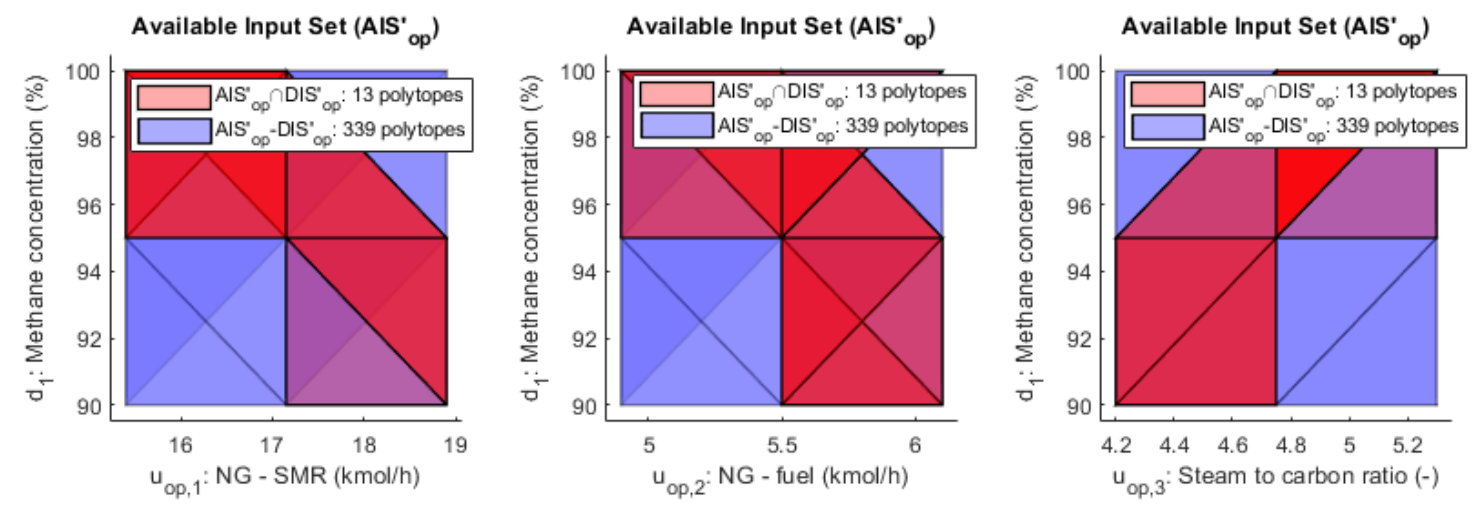

Figure 49. Projections of AIS' for the design $u_{\text {des }}=(16,11)$.
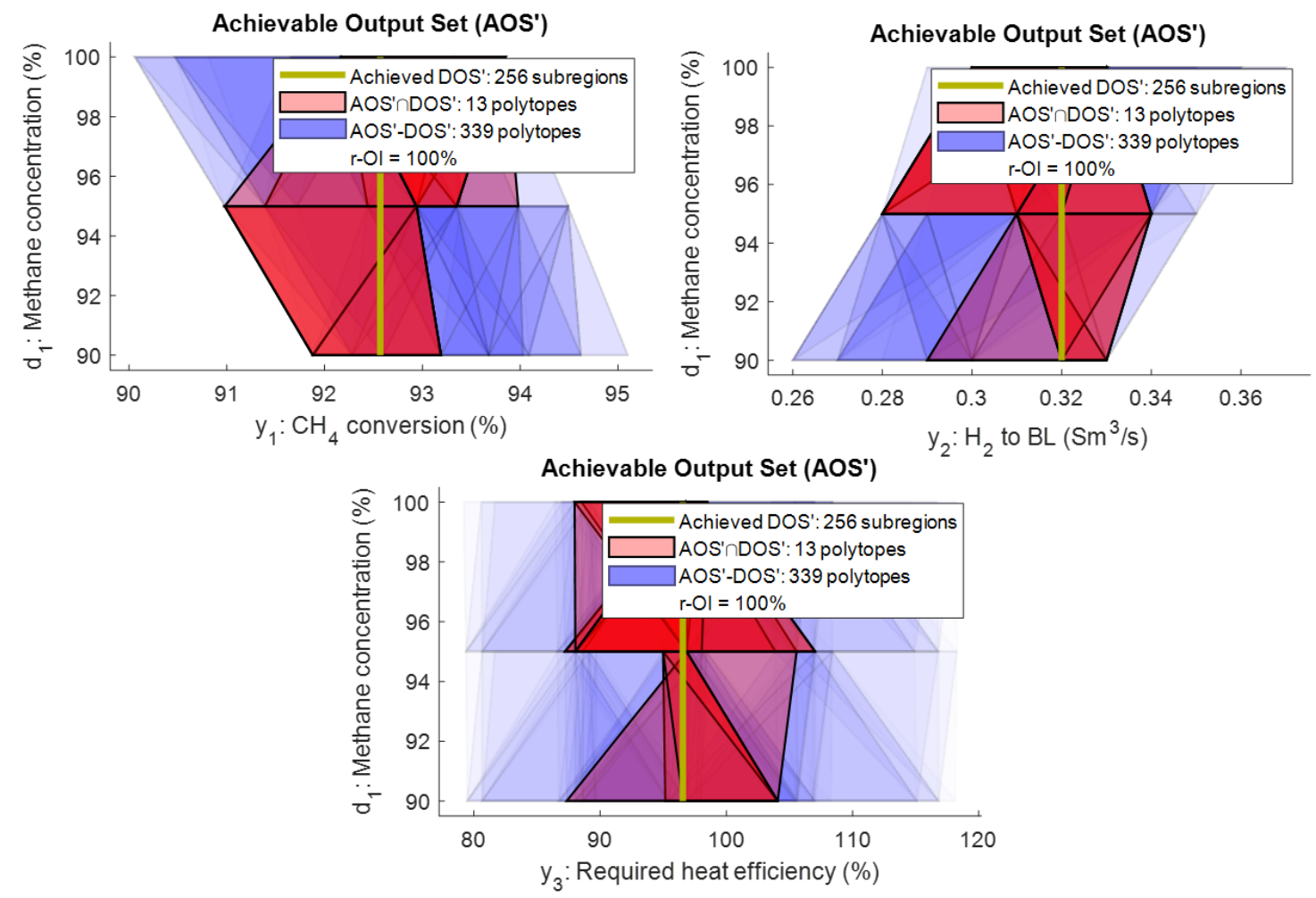

Figure 50. Projections of AOS' for the design $u_{d e s}=(16,11)$. 
Figures 51 and 52 show the projections for the design $u_{\text {des }}=(23,20)$. Similarly, Figures 51 and 52 contain projected polytopes according to the specific values of methane concentration. In this case, the considered design is not flexible enough to keep the system at the desired setpoint in any disturbance scenario. The lower number of connected polytopes is a result of infeasibilities in the operation of this design. The simulator log indicates temperature cross in at least one of the heat exchangers, and the multimodels associated with such infeasible operation are not included. The nonempty intersection between projections of AOS' and DOS' in Figure 52 indicate that certain CVs can be achieved individually, but never simultaneously. Note that the required heat efficiency $y_{3}^{N}=96.56 \%$ is unachievable for all disturbance scenarios. Therefore, there are no combinations $\left(y_{1}, y_{2}, y_{2}\right) \in A O S$ that achieve the setpoint $y^{N}$ at any disturbance scenario.
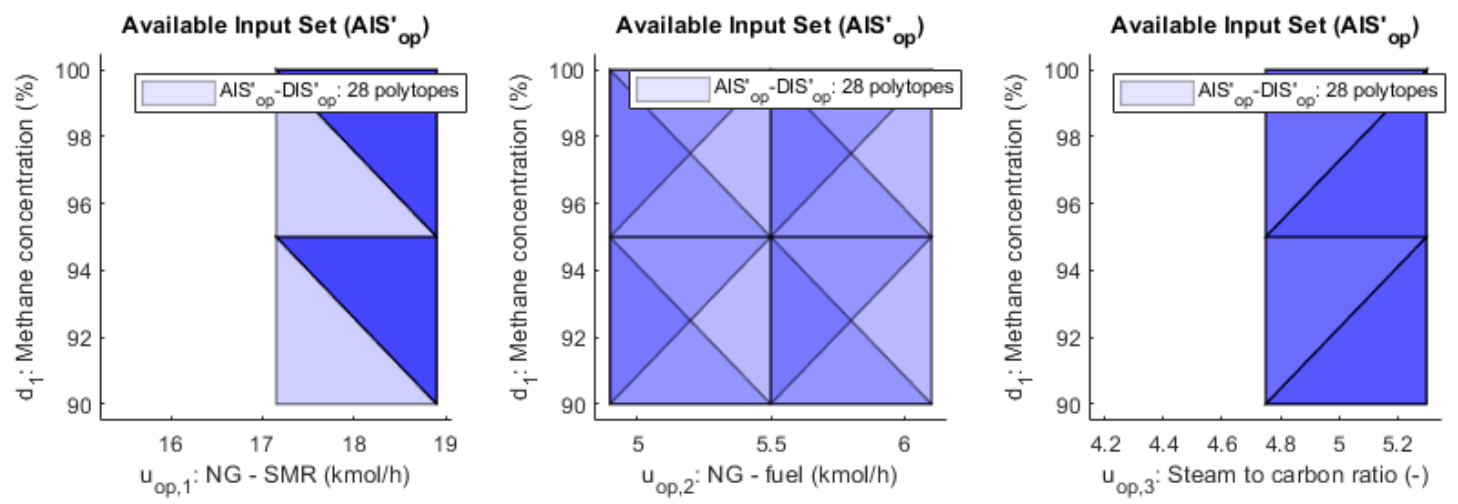

Figure 51. Projections of AIS' for the design $u_{d e s}=(23,20)$. 

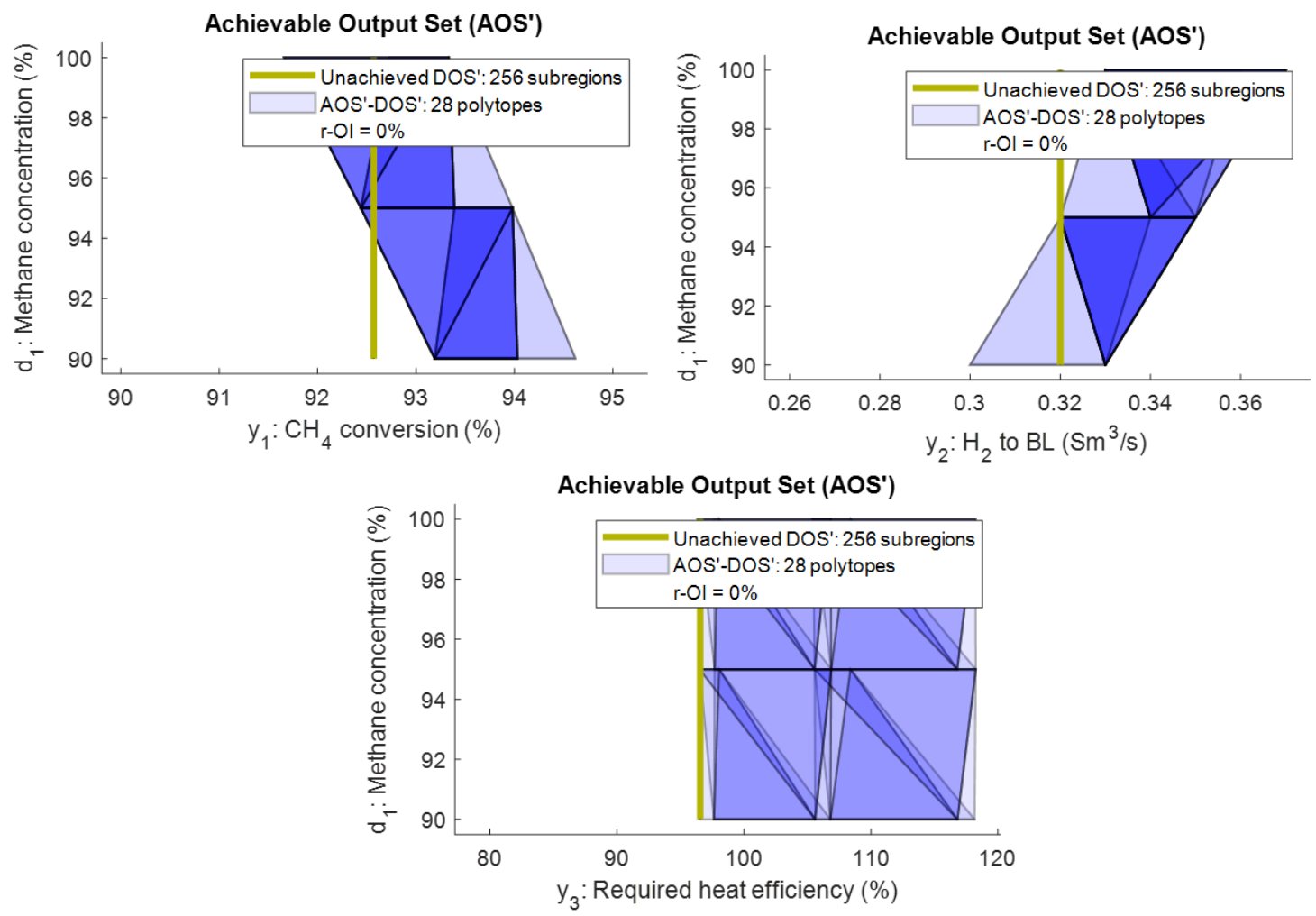

Figure 52. Projections of AOS' for the design $u_{d e s}=(23,20)$.

The analysis of $\mathrm{r}$-Ol indicates that smaller heat exchangers should be further investigated. The main consequence of choosing small areas for E-104 is that, at certain situations, more energy is required at the steam boiler since less heat is recuperated from the process to the steam side. The prescreening activity indicated that enough tail gas is produced to fuel the steam boiler, counterbalancing the disadvantage of a lower heat exchanger area. A smaller area for E-106, however, could require an abnormal amount of cooling water. Further studies should analyze the tradeoff between employed flowrates of cooling water and heat exchanger area, with a focus on total cost and constraints associated with water knockout at the flash drum. 


\section{Chapter 8 Conclusion and Recommendations}

\subsection{Conclusions}

A multimodel operability framework was presented for the design of emerging technologies characterized by SM and PI. This framework is flexible and could also be extended to attain other goals such as cost, environmental targets, capacity, purity, and so on. The representation of the nonlinear system by linearized models, geometrically represented by polytopes, was systematically applied for the calculation of an optimal design and operability assessment to ensure feasibility, flexibility and optimally.

The developed framework shows unique features for tackling energy system applications whose designs are challenged by dimensionality, process model nonlinearities and highly constrained environments. The use of the $\mathrm{Ol}$ as a measure for raking competing designs in SM and $\mathrm{PI}$ literature was explored in this study for the first time. The obtained results showed that calculation of optimal modular designs, the analysis of achievability and flexibility, and the selection of feasible operation. It was noted that energy system designs that optimally fulfill PI and SM considering a fixed nominal operation do not necessarily ensure system operability.

The state-of-the-art process operability algorithms were further developed and adapted to be compiled as part of an open-source operability platform. This platform grants the process systems community access to operability approaches and algorithms, motivating the further dissemination of these algorithms and improvements in the process operability field. The developed Operability App in MATLAB corresponds to the first effort to include contributions of other researchers that have worked in operability in the past or intend to do in the future.

Existing TEA methods were extended for costing of intensified modular systems. Adaptations of capital and operating costs were allowed with a focus on high degrees of process integration and customization. An economy of learning model was incorporated to include the effect of an experience curve in purchase price, as modular technology matures. 
The developed modular TEA framework selected the parameters that were economically relevant for modular systems deployment. Economic cost indicators were scaled with respect to production of a chemical of interest. Then, a comparative analysis of profitability was allowed for small scale modular capacity. The scaled measures of cost were used to evaluate profitability of individual modular units and of an assembled modular plant. For both cases, competitive scenarios were mapped, showing in which scenarios the modular process broke even with respect to the conventional counterpart.

The addressed applications showed versatility of the developed framework and software tools. The comparison of measures of $\mathrm{Ol}$ in terms of subregions and hypervolumes indicated that specific cases may not be compatible to the original interpretation of the $\mathrm{Ol}$ in terms of hypervolumes, being suitable for the measure using subregions. Methods for selecting operating regions were used to determinate designs and nominal operations that achieve SM and PI targets while respecting process constraints.

In the DMA-MRA applications, comparison between the distinct tasks of minimizing footprint to achieve SM while respecting PI targets and maximizing process operability represented by the $\mathrm{OI}$, showed that these two objectives might be conflicting. Small design changes were also performed to ensure achievability of a region of nominal points. Later, this analysis was extended to study system flexibility in terms of achieving a nominal point considering expected disturbance scenarios.

All presented results were generated using the developed Process Operability App, demonstrating the app adaptability to systems of various natures and dimensionalities. Particularly, the CCS application associated with the coal-fired power plant cycling demonstrated how reduced-order or surrogate models can be employed for achievability analysis, generating meaningful results without necessarily employing $\mathrm{OI}$ calculations. The application of NLP-based approaches to the DMA-MR process showed how previously developed concepts were also incorporated to deal with the challenge of infeasibilities.

A modular SMR unit application was developed, considering an intensified microchannel reactor. Process synthesis resulted in a highly integrated modular process. 
Process operability was used to find feasible operation of the obtained process. The modular TEA framework application to the developed process indicated that the modular hydrogen unit can be profitable in comparison with a conventional SMR plant for scenarios in which NG price is under $0.02 \mathrm{US} \$ / \mathrm{Sm}^{3}$ and the economy of learning produces a reduction of about $40 \%$ in purchase cost. The study of contingency and fees showed that the learning phenomenon can also benefit modular deployment by providing more reliable cost data. The assembly of a modular SMR plant that consisted of 92 modular hydrogen units was considered for comparison at conventional industrial scale. Combinations of required experience curve parameters were obtained to achieve breakeven cost according to NG price. The economy of learning had to follow less aggressive scenarios if $N G$ is cheap. At market prices, reductions in purchase cost needed to be drastic, and therefore, unrealistic. This result suggests the modular SMR units are promising in regions with abundant offer of NG, where it is usually flared or reinjected to the reservoir. Remote NG and shale gas formations also present potential for the modular hydrogen units, as conventional SMR plants can't usually be installed at these locations due to lack of infrastructure.

\subsection{Recommendations and Future Work}

Application of the developed process operability framework is recommended for design of nonlinear energy systems at early stages of technology development. Design retrofit and management of process operations can also benefit from the developed framework. Adaptations of the employed objective functions and production goals can be performed for applications beyond the scope of SM and PI.

The presented modular TEA framework identifies scenarios in which modular technologies are profitable and competitive. It is also a tool for guiding the deployment and manufacturing of modular systems, providing instructions for management of the experience curve. 
Recommendations regarding future research work are the following:

(1) The development of a multi-objective optimization-based operability that can analyze tradeoffs between maximization of the $\mathrm{Ol}$ measure and other conflicting objectives such as footprint minimization and cost.

(2) The modular TEA framework could be further integrated with process operability.

(3) The EDS could allow operability to be a tool for interfacing design and control under uncertainty. For that, incorporation of the process stochastic behavior is recommended. ${ }^{76}$

(4) Extensions are suitable to the MILP-based algorithms, such as the examination of other ways to increase space resolution and the adaptation to mixed-integer nonlinear programming (MINLP)-based formulations.

(5) Other optimization-based algorithms can be formulated to provide an unbiased selection of the DOS. Examples are particle swarm optimization and trust-region methods.

(6) Both the multimodel and NLP-based process operability approaches would benefit from other input-output system representations, especially for the representation of high-dimensional processes.

(7) Investigation on the upper limit in the capability of the employed algorithms is recommended for both multimodel and NLP-based in terms of dimensionalities and process model complexity.

(8) Inclusion of other process operability algorithms associated with process dynamics and interval control would increase the range of application of the Process Operability App.

(9) Consideration of systems that must be operated around singularities or discontinuous spaces are highlighted as potential critical limitations for the evaluation of existing operability algorithms.

(10) Both dynamic and steady-state process operability concepts could be integrated with operator advisory systems, enabling the future improvement of plant operations.

(11) There is an opportunity to integrate process operability with real-time model-based control and optimization algorithms such as model predictive control (MPC) and dynamic real-time optimization (DRTO). 


\section{References}

(1) Bishop, B. A.; Lima, F. V. Novel Module-Based Membrane Reactor Design Approach for Improved Operability Performance. Membranes. 2021, 11 (2), 1-18. https://doi.org/10.3390/membranes11020157.

(2) Vinson, D. R.; Georgakis, C. New Measure of Process Output Controllability. J. Process Control 2000. https://doi.org/10.1016/S0959-1524(99)00045-1.

(3) Gazzaneo, V.; Carrasco, J. C.; Vinson, D. R.; Lima, F. V. Process Operability Algorithms: Past, Present, and Future Developments. Ind. Eng. Chem. Res. 2020. https://doi.org/10.1021/acs.iecr.9b05181.

(4) Gazzaneo, V.; Lima, F. V. Multilayer Operability Framework for Process Design, Intensification, and Modularization of Nonlinear Energy Systems. Ind. Eng. Chem. Res. 2019. https://doi.org/10.1021/acs.iecr.8b05482.

(5) Gazzaneo, V.; Carrasco, J. C.; Lima, F. V. An MILP-Based Operability Approach for Process Intensification and Design of Modular Energy Systems. In Computer Aided Chemical Engineering; 2018. https://doi.org/10.1016/B978-0-444-642417.50390-6.

(6) Vinson, D. R.; Georgakis, C. Inventory Control Structure Independence of the Process Operability Index. Ind. Eng. Chem. Res. 2002. https://doi.org/10.1021/ie0109814.

(7) Uztürk, D.; Georgakis, C. Inherent Dynamic Operability of Processes: General Definitions and Analysis of SISO Cases. Ind. Eng. Chem. Res. 2002. https://doi.org/10.1021/ie0101792. 
(8) Lima, F. V.; Georgakis, C. Dynamic Operability for the Calculation of Transient Output Constraints for Non-Square Linear Model Predictive Controllers. In IFAC Proceedings Volumes (IFAC-PapersOnline); 2009.

(9) Lima, F. V.; Jia, Z.; lerapetritou, M.; Georgakis, C. Similarities and Differences between the Concepts of Operability and Flexibility: The Steady-State Case. AIChE J. 2010, 56 (3), 702-716. https://doi.org/10.1002/aic.12021.

(10) Georgakis, C.; Uztürk, D.; Subramanian, S.; Vinson, D. R. On the Operability of Continuous Processes. Control Eng. Pract. 2003. https://doi.org/10.1016/S09670661(02)00217-4.

(11) Davis, E.; lerapetritou, M. A Kriging Method for the Solution of Nonlinear Programs with Black-Box Functions. AIChE J. 2007. https://doi.org/10.1002/aic.11228.

(12) Boukouvala, F.; Muzzio, F. J.; lerapetritou, M. G. Design Space of Pharmaceutical Processes Using Data-Driven-Based Methods. J. Pharm. Innov. 2010. https://doi.org/10.1007/s12247-010-9086-y.

(13) Georgakis, C. Design of Dynamic Experiments: A Data-Driven Methodology for the Optimization of Time-Varying Processes. Ind. Eng. Chem. Res. 2013. https://doi.org/10.1021/ie3035114.

(14) Kiparissides, A.; Georgakis, C.; Mantalaris, A.; Pistikopoulos, E. N. Design of in Silico Experiments as a Tool for Nonlinear Sensitivity Analysis of Knowledge-Driven Models. Ind. Eng. Chem. Res. 2014. https://doi.org/10.1021/ie4032154.

(15) Swaney, R. E.; Grossmann, I. E. An Index for Operational Flexibility in Chemical 
Process Design. Part II: Computational Algorithms. AlChE J. 1985, 31 (4), 631641. https://doi.org/10.1002/aic.690310413.

(16) Grossmann, I. E.; Floudas, C. A. Active Constraint Strategy for Flexibility Analysis in Chemical Processes. Comput. Chem. Eng. 1987, 11 (6), 675-693. https://doi.org/10.1016/0098-1354(87)87011-4.

(17) Carrasco, J. C.; Lima, F. V. Novel Operability-Based Approach for Process Design and Intensification: Application to a Membrane Reactor for Direct Methane Aromatization. AIChE J. 2017. https://doi.org/10.1002/aic.15439.

(18) Carrasco, J. C.; Lima, F. V. Bilevel and Parallel Programing-Based Operability Approaches for Process Intensification and Modularity. AlChE J. 2018. https://doi.org/10.1002/aic.16113.

(19) Goyal, V.; lerapetritou, M. G. Determination of Operability Limits Using Simplicial Approximation. A/ChE J. 2002. https://doi.org/10.1002/aic.690481217.

(20) Zukui, L.; lerapetritou, M. G. A New Methodology for the General Multiparametric Mixed-Integer Linear Programming (MILP) Problems. Ind. Eng. Chem. Res. 2007. https://doi.org/10.1021/ie070148s.

(21) Pistikopoulos, E. N.; Diangelakis, N. A. Towards the Integration of Process Design, Control and Scheduling: Are We Getting Closer? Comput. Chem. Eng. 2016. https://doi.org/10.1016/j.compchemeng.2015.11.002.

(22) Georgakis, C.; Li, L. On the Calculation of Operability Sets of Nonlinear HighDimensional Processes. Ind. Eng. Chem. Res. 2010. 
https://doi.org/10.1021/ie1009316.

(23) Zheng, C.; Zhao, F.; Zhu, L.; Chen, X. Flexibility Index and Design of Chemical Systems by Cylindrical Algebraic Decomposition. Comput. Chem. Eng. 2021, 144, 107142. https://doi.org/10.1016/j.compchemeng.2020.107142.

(24) Zhao, F.; Chen, X. Analytical and Triangular Solutions to Operational Flexibility Analysis Using Quantifier Elimination. AIChE J. 2018, 64 (11), 3894-3911. https://doi.org/10.1002/aic.16207.

(25) Zhao, F.; Zheng, C.; Zhang, S.; Zhu, L.; Chen, | Xi. Quantification of Process Flexibility via Space Projection. AlChE J. 2019, 65 (10). https://doi.org/10.1002/aic.16706.

(26) Adi, V. S. K.; Laxmidewi, R. Design and Operability Analysis of Membrane Module Based on Volumetric Flexibility. In Computer Aided Chemical Engineering; Elsevier B.V., 2017; Vol. 40, pp 1231-1236. https://doi.org/10.1016/B978-0-444-639653.50207-5.

(27) Adi, V. S. K.; Laxmidewi, R.; Chang, C. T. An Effective Computation Strategy for Assessing Operational Flexibility of High-Dimensional Systems with Complicated Feasible Regions. Chem. Eng. Sci. 2016, 147, 137-149. https://doi.org/10.1016/j.ces.2016.03.028.

(28) Konge, U.; Baikadi, A.; Mondi, J.; Subramanian, S. Data-Driven Model Based Computation and Analysis of Operability Sets Using High-Dimensional Continuation: A Plant-Wide Case Study. Ind. Eng. Chem. Res. 2020, 59 (21), 10043-10060. https://doi.org/10.1021/acs.iecr.9b07087. 
(29) Bahri, P. A.; Bandoni, J. A.; Romagnoli, J. A. Integrated Flexibility and Controllability Analysis in Design of Chemical Processes. AlChE J. 1997, 43 (4), 997-1015. https://doi.org/10.1002/aic.690430415.

(30) Solovyev, B. M.; Lewin, D. R. A Steady-State Process Resiliency Index for Nonlinear Processes: 1. Analysis. Ind. Eng. Chem. Res. 2003, 42 (20), 4506-4511. https://doi.org/10.1021/ie020926a.

(31) Abubakar, U.; Sriramula, S.; Renton, N. C. Reliability of Complex Chemical Engineering Processes. Comput. Chem. Eng. 2015, 74, 1-14. https://doi.org/10.1016/j.compchemeng.2014.12.013.

(32) Heckel, J. P.; Becker, C. Dynamic Simulation of an Integrated Energy System for Northern Germany with Improved Resilience. ETG-Kongress 2019 - Das Gesamtsystem im Fokusder Energiewende 2019, 165-170.

(33) Grossmann, I. E.; Calfa, B. A.; Garcia-Herreros, P. Evolution of Concepts and Models for Quantifying Resiliency and Flexibility of Chemical Processes. Comput. $\begin{array}{llll}\text { Chem. } & \text { 2014, } & \text { 22-34. }\end{array}$ https://doi.org/10.1016/j.compchemeng.2013.12.013.

(34) Ulbig, A.; Andersson, G. Analyzing Operational Flexibility of Electric Power Systems. Int. J. Electr. Power Energy Syst. 2015, 72, 155-164. https://doi.org/10.1016/j.ijepes.2015.02.028.

(35) Di Pretoro, A.; Montastruc, L.; Joulia, X.; Manenti, F. Accounting for Dynamics in Flexible Process Design: A Switchability Index. Comput. Chem. Eng. 2021, 145, 107149. https://doi.org/10.1016/j.compchemeng.2020.107149. 
(36) Dimitriadss, V. D.; Pistikopoulos, E. N. Flexibility Analysis of Dynamic Systems; 1995; Vol. 34. https://doi.org/10.1021/ie00039a036.

(37) Lima, F. V.; Georgakis, C. Design of Output Constraints for Model-Based NonSquare Controllers Using Interval Operability. J. Process Control 2008. https://doi.org/10.1016/j.jprocont.2007.09.004.

(38) Heavy Haul Trucking. Tractor Trailer Dimensions http://www.heavyhaul.net/tractortrailer-dimensions/ (accessed May 10, 2018).

(39) Road Weight and Size limitations http://www.cargoagents.net/resources/ stateroadweightsizelimitations.htm (accessed May 10, 2018).

(40) Haque, A., et al. CHE 455: Senior Design Report; West Virginia University; 2018.

(41) Baldea, M.; Edgar, T. F.; Stanley, B. L.; Kiss, A. A. Modular Manufacturing Processes: Status, Challenges, and Opportunities. AlChE J. 2017. https://doi.org/10.1002/aic.15872.

(42) Carrasco, J. C.; Lima, F. V. Operability-Based Approach for Process Design, Intensification, and Control: Application to High-Dimensional and Nonlinear Membrane Reactors. In FOCAPO/CPC; 2017.

(43) Carrasco, J. C.; Lima, F. V. An Optimization-Based Operability Framework for Process Design and Intensification of Modular Natural Gas Utilization Systems. Comput. Chem. Eng. 2017. https://doi.org/10.1016/j.compchemeng.2016.12.010.

(44) Tian, Y.; Pistikopoulos, E. N. Synthesis of Operable Process Intensification Systems - Steady-State Design with Safety and Operability Considerations. Ind. 
Eng. Chem. Res. 2019, $58 \quad$ (15), 6049-6068. https://doi.org/10.1021/acs.iecr.8b04389.

(45) Tian, Y.; Sam Mannan, M.; Pistikopoulos, E. N. Towards a Systematic Framework for the Synthesis of Operable Process Intensification Systems. In Computer Aided Chemical Engineering; Elsevier B.V., 2018; Vol. 44, pp 2383-2388. https://doi.org/10.1016/B978-0-444-64241-7.50392-X.

(46) Tian, Y.; Sam Mannan, M.; Kravanja, Z.; Pistikopoulos, E. N. Towards the Synthesis of Modular Process Intensification Systems with Safety and Operability Considerations - Application to Heat Exchanger Network. In Computer Aided Chemical Engineering; Elsevier B.V., 2018; Vol. 43, pp 705-710. https://doi.org/10.1016/B978-0-444-64235-6.50125-X.

(47) Tian, Y.; Pappas, I.; Burnak, B.; Katz, J.; Pistikopoulos, E. N. A Systematic Framework for the Synthesis of Operable Process Intensification Systems Reactive Separation Systems. Comput. Chem. Eng. 2020, 134, 106675. https://doi.org/10.1016/j.compchemeng.2019.106675.

(48) Demirel, S. E.; Li, J.; Hasan, M. M. F. Systematic Process Intensification Using Building Blocks. Comput. Chem. 2017. https://doi.org/10.1016/j.compchemeng.2017.01.044.

(49) Demirel, S. E.; Li, J.; Hasan, M. M. F. A General Framework for Process Synthesis, Integration, and Intensification. Ind. Eng. Chem. Res. 2019, 58 (15), 5950-5967. https://doi.org/10.1021/acs.iecr.8b05961.

(50) Iyer, S. S.; Bajaj, I.; Balasubramanian, P.; Hasan, M. M. F. Integrated Carbon 
Capture and Conversion to Produce Syngas: Novel Process Design, Intensification, and Optimization. Ind. Eng. Chem. Res. 2017. https://doi.org/10.1021/acs.iecr.7b01688.

(51) Tula, A. K.; Eden, M. R.; Gani, R. ProCAFD: Computer-Aided Tool for Sustainable Process Synthesis, Intensification and Hybrid Solutions. In Computer Aided Chemical Engineering; Elsevier B.V., 2019; Vol. 46, pp 481-486. https://doi.org/10.1016/B978-0-12-818634-3.50081-3.

(52) Grant, M. R. Contemporary Strategy Analysis, 10th ed.; Wiley, 2018.

(53) Yelle, L. E. THE LEARNING CURVE: HISTORICAL REVIEW AND COMPREHENSIVE SURVEY. Decis. Sci. 1979. https://doi.org/10.1111/j.15405915.1979.tb00026.x.

(54) Quarterman, L. P. E. Learning \&amp; Experience Curves In Manufacturing; 2014.

(55) Lier, S.; Grünewald, M. Net Present Value Analysis of Modular Chemical Production Plants. Chem. Eng. Technol. 2011. https://doi.org/10.1002/ceat.201000380.

(56) Boldon Lauren M.; Piyush, S. Small Modular Reactor: First-of-a-Kind (FOAK) and Nth-of-a-Kind (NOAK) Economic Analysis; Idaho Falls, ID (United States), 2014.

(57) Kuznetsov, V. Options for Small and Medium Sized Reactors (SMRs) to Overcome Loss of Economies of Scale and Incorporate Increased Proliferation Resistance and Energy Security. Prog. Nucl. Energy 2008. https://doi.org/10.1016/j.pnucene.2007.11.006. 
(58) Herceg, M.; Kvasnica, M.; Jones, C.; Morari, M. Multi-Parametric Toolbox 3.0. In Proceedings of the European Control Conference; 2013. https://doi.org/10.23919/ECC.2013.6669862.

(59) Li, L.; Borry, R. W.; Iglesia, E. Design and Optimization of Catalysts and Membrane Reactors for the Non-Oxidative Conversion of Methane. Chem. Eng. Sci. 2002. https://doi.org/10.1016/S0009-2509(02)00314-7.

(60) Li, J.; Yoon, H.; Wachsman, E. D. Hydrogen Permeation through Thin Supported SrCe0.7Zr0.2Eu0.1O3-ס Membranes; Dependence of Flux on Defect Equilibria and $\begin{array}{lllll}\text { Operating } & \text { Conditions. } & \text { J. } & \text { Memb. } & \text { Sci. }\end{array} 2011$. https://doi.org/10.1016/j.memsci.2011.07.032.

(61) Carrasco, J. C.; Lima, F. V. Nonlinear Operability of a Membrane Reactor for Direct Methane Aromatization. In IFAC-PapersOnLine; 2015. https://doi.org/10.1016/j.ifacol.2015.09.055.

(62) Rawlings, J.B. and Ekerdt, J. G. Chemical Reactor Analysis and Design Fundamentals; Nob Hill Publishing, Madison, WI, USA, 2002.

(63) EIA. U.S. Energy Information Administration: Drilling Productivity Report https://www.eia.gov/petroleum/drilling/pdf/dpr-full.pdf (accessed Feb 28, 2019).

(64) Marcellus Shale Energy and Environment Laboratory. Well Production http://www.mseel.org/ (accessed Feb 28, 2019).

(65) He, X.; Lima, F. V. Development and Implementation of Advanced Control Strategies for Power Plant Cycling with Carbon Capture. Comput. Chem. Eng. 
2019. https://doi.org/10.1016/j.compchemeng.2018.11.004.

(66) Kim, R.; Lima, F. V. A Tchebycheff-Based Multi-Objective Combined with a PSOSQP Dynamic Real-Time Optimization Framework for Cycling Energy Systems. Chem. Eng. Res. Des. 2020, 156, 180-194. https://doi.org/10.1016/j.cherd.2020.01.020.

(67) Carrasco, J. C. Operability-Based Design of Energy Systems: Application to Natural Gas Utilization Processes, West Virginia University, 2017.

(68) Bailie, R. C.; Whiting, W. B.; Shaeiwitz, J. A.; Turton, R.; Bhattacharyya, D. Analysis, Synthesis, and Design of Chemical Processes, Fifth Edit.; 2018.

(69) Henderson, B. D. The Experience Curve-Reviewed (Part II) https://www.bcg.com/publications/1973/corporate-finance-strategy-portfoliomanagement-experience-curve-reviewed-part-ii-the-history (accessed Mar 11, 2021).

(70) Tonkovich, A. Y.; Perry, S.; Wang, Y.; Quu, D.; Laplante, T.; Rogers, W. A. MicroChannel Process Technology for Compact Methane Steam Reforming. In Chemical Engineering Science; 2004. https://doi.org/10.1016/j.ces.2004.07.098.

(71) Tonkovich, A.; Fitzgerald, S.; Neagle, P.; ... D. Q.-U. P.; 2007, undefined. (12) United States Patent (45) Date of Patent: (54) (75) (73) (*) (21) (22) (65) (51) (52) (58) METHODS OF CONDUCTING SMULTANEOUS ENDOTHERMC AND EXOTHERMC REACTIONS; 2007.

(72) Collodi, G.; Azzaro, G.; Ferrari, N.; Santos, S. Techno-Economic Evaluation of 
Deploying CCS in SMR Based Merchant H2 Production with NG as Feedstock and Fuel. In Energy Procedia; 2017. https://doi.org/10.1016/j.egypro.2017.03.1533.

(73) Towler, G.; Sinnott, R. K. Chemical Engineering Design - Principles, Practice and Economics of Plant and Process Design (2nd Edition); 2013.

(74) Kelly, I. Equipment Design and Cost Estimation for Small Modular Biomass Systems, Synthesis Gas Cleanup, and Oxygen Separation Equipment; 2006.

(75) Occupational Employment Statistics https://beta.bls.gov/dataViewer/view/ timeseries/OEUM001662000000051809104 (accessed Aug 11, 2020).

(76) de Araujo, W. R.; Lima, F. V.; Bispo, H. Dynamic and Statistical Operability of an Experimental Batch Process. Processes 2021, 9 (3), 1-19. https://doi.org/10.3390/pr9030441. 


\section{Appendix A. Modular SMR - Process Model}

Process synthesis of the modular hydrogen unit is carried out around the microchannel reactor. Therefore, preconditioning of feedstocks and parts of the process are designed according to literature indication regarding reactor requirements. ${ }^{70}$ Unit operations are synthesized to adequate reactor inlets, knock water out from the products, purify hydrogen and integrate steam generation to the process.

At first, boundary limit conditions associated with raw materials, utilities and produced substances are considered, as follows: (a) $\mathrm{NG}$ at $25^{\circ} \mathrm{C}$ and $3.1 \mathrm{MPa}$, composed by molar fractions of $97.6 \%$ of $\mathrm{CH}_{4}, 1.8 \%$ of $\mathrm{CO}_{2}, 0.4 \%$ of $\mathrm{C}_{2} \mathrm{H}_{6}, 0.3 \%$ of $\mathrm{N}_{2}$ and a trace of other impurities; (b) Air is obtained at $25{ }^{\circ} \mathrm{C}$ and atmospheric pressure, composed by molar fractions of $79 \%$ of $\mathrm{N}_{2}$ and $21 \%$ of $\mathrm{O}_{2}$; (c) High-purity water for process use at 25 ${ }^{\circ} \mathrm{C}$ and $0.175 \mathrm{MPa}$; (d) Flue gas with temperature and pressures ranges of $120-176 \stackrel{\circ}{\circ}$ and $0.152-0.175 \mathrm{MPa}$ respectively; and (e) Hydrogen at $47{ }^{\circ} \mathrm{C}$ and $1.261 \mathrm{MPa}$, composed by molar fractions higher than $99.9 \%$ of hydrogen and impurities.

Then, the microchannel reactor is scaled-up and modeled in Aspen Plus. The following modeling assumptions are considered: (a) the amount of $10.7 \%$ of the total heat produced by combustion microchannels is used as a finishing preheating step of air and fuel mixture; (b) the combustion microchannel is simplified to a model based on reaction conversion and the produced heat is integrated with the reaction microchannel; (c) the amount of inlet air is controlled to be proportional to 9.57 times the intake of NG in the combustion microchannel; (d) reaction microchannels are operated at an average of 811 ${ }^{\circ} \mathrm{C}$. The microchannel reactor is validated with inlets and outlets that are consistent in composition, temperature and pressure with experimental data from literature. ${ }^{70}$

The main modular hydrogen process assumptions are related to the steam generation. The adopted assumptions are the following: (a) the steam boiler is operated with $10 \%$ of air excess; (b) there is a blowdown proportional to $10 \%$ of all circulated water; (c) the deaerator is fed with low-pressure steam in a proportion of $0.64 \%$ of its total inlet, and it is modeled as flash vessel with pressure drop of 0.05 bar; (d) the PSA has an efficiency of $90 \%$, and produces a hydrogen steam with purity higher than $99.9 \%$; and (e) PSA and desulfurization beds are operated continuously in a swing operation mode. Let- 
down valves for pressure relieve are omitted in the flowsheet representation. Flowrate specifications for the main process streams are included in Table A.1 below. The stream identification follows the convention from Figure 36.

Table A.1. Description of main process streams.

\begin{tabular}{|c|c|c|c|c|c|c|c|c|c|c|}
\hline Id. & 1 & 2 & 3 & 4 & 5 & 6 & 7 & 8 & 9 & 10 \\
\hline $\bar{T}\left({ }^{\circ} \mathrm{C}\right)$ & 17 & $\begin{array}{l}10.4 \\
\end{array}$ & 160 & 305 & 198.5 & 340 & 355 & 47 & 47 & 25.5 \\
\hline $\mathrm{P}(\mathrm{MPa})$ & 1.5 & 0.3 & 0.3 & 1.5 & 1.5 & 1.3 & 0.2 & 1.3 & 1.3 & 0.1 \\
\hline \multicolumn{11}{|c|}{ Molar flowrate $(\mathrm{mol} / \mathrm{hr})$} \\
\hline $\mathrm{N}_{2}$ & 72 & 18 & 41718 & 54 & 0 & 54 & 41735 & 54 & 1 & 54 \\
\hline $\mathrm{O}_{2}$ & 0 & 0 & 11090 & 0 & 0 & 0 & 685 & 0 & 0 & 0 \\
\hline $\mathrm{CH}_{4}$ & $\begin{array}{c}221 \\
12\end{array}$ & 5383 & 0 & 16728 & 0 & 1242 & 215 & 1242 & 0 & 1242 \\
\hline $\mathrm{CO}$ & 0 & 0 & 0 & 0 & 0 & 7615 & 0 & 7615 & 0 & 7615 \\
\hline $\mathrm{H}_{2} \mathrm{O}$ & 0 & 0 & 0 & 81647 & 81647 & 58290 & 10395 & 547 & 0 & 547 \\
\hline $\mathrm{CO}_{2}$ & 401 & 98 & 0 & 303 & 0 & 8174 & 5305 & 8173 & 0 & 8173 \\
\hline $\mathrm{H}_{2}$ & 0 & 0 & 0 & 0 & 0 & 54330 & 0 & 54330 & 48897 & 5433 \\
\hline $\mathrm{C}_{2} \mathrm{H}_{8}$ & 80 & 20 & 0 & 61 & 0 & 61 & 0 & 61 & 0 & 61 \\
\hline
\end{tabular}

$\mathrm{T}$ and $\mathrm{P}$ indicate temperature and pressure, respectively. 


\section{Appendix B. Modular SMR - Equipment Cost Calculation}

Extension of conventional costing techniques are applied to estimate the bare module cost of the modular process. ${ }^{68}$ Heat exchangers, microchannel reactor, steam boiler and flash drum have the purchase cost estimated by extrapolating capacity vs. cost curves. The deaerator and the PSA and desulfurization units have the purchase cost extracted from the literature. The bare module cost of each piece of equipment is estimated considering the values of purchase cost and customization from material and adopted design conditions.

When the purchase cost is estimated by extrapolating capacity vs. cost curves, Equation (86) is applied.

$$
\log _{10} C_{p}^{0}=K_{1}+K_{2} \log _{10}(A)+K_{3}\left[\log _{10}(A)\right]^{2}
$$

where $C_{p}^{0}$ is the purchase cost at base conditions (carbon steel material and atmospheric pressure), $A$ is the equipment capacity and $K_{1}, K_{2}$, and $K_{3}$ are constants.

The values of constraints $K$ is obtained from ref. ${ }^{68}$ by informing the specific type of equipment. Heat exchangers are considered of type "floating head"; microchannel reactor, a furnace of type a "reformer furnace"; steam boiler, a heater of type "steam boiler"; flash drum, vessel of type "vertical process vessel". Attribute $A$ is area, heat duty and volume for heat exchangers, microchannel reactor and steam boiler, and flash drum respectively.

For the deaerator, PSA and desulfurization units, equipment specification and total inlet flowrates from ref. ${ }^{74}$ are used with the six-tenths rule represented by Equation (87).

$$
C_{p}^{r}=C_{r}\left(\frac{A}{A_{r}}\right)^{0.6}
$$

where $C_{p}^{r}$ is the adjusted purchase cost at the reference conditions, $C_{r}$ is the purchase cost from the reference, and $A$ and $A_{r}$ are the capacities of the considered process and the reference, respectively. 
In Table 8, values of $A$ for all pieces of equipment are included. The application of Equations (86) and (87) allow the extrapolation of cost beyond original bounds for capacity $A$.

Once purchase costs are obtained at base conditions, the bare module cost is calculated. Bare module costs are more comprehensive than purchase cost and include direct (equipment, materials and labor) and indirect (freight, overhead and engineering) costs. The methodology from ref. ${ }^{68}$ is repeated to consider material, operating conditions and remaining cost. Carbon steel is considered for the deaerator, while stainless steel is considered for the rest of the equipment.

For the equipment costed with Equation (86), the same equipment type is used to apply corrections from methodology from from ref. ${ }^{68}$. For the equipment costed with Equation (87), type "vertical process vessel" is used to obtain the bare module cost. Since both reference and the developed process have the same materials and conditions, the deaerator and the PSA and desulfurization units do not include corrections associated with these factors. Table B.1 shows the pressures used to correct cost, number of units per process and the obtained values of purchase and bare module cost. 
Table B.1. Estimation of purchase and bare module costs.

\begin{tabular}{ccccc}
\hline Id. & Quantity & $\begin{array}{c}\text { Pressure } \\
\text { (barg) }\end{array}$ & $\begin{array}{c}\text { Purchase } \\
\text { cost }\end{array}$ & $\begin{array}{c}\text { Bare module } \\
\text { cost }\end{array}$ \\
\hline \hline E-101 & 1 & 1.8 & US $\$ 26,026$ & US $\$ 160,366$ \\
E-102 & 1 & 14.0 & US $\$ 25,683$ & US $\$ 162,922$ \\
E-103 & 1 & 14.0 & US $\$ 31,329$ & US $\$ 198,737$ \\
E-104 & 1 & 14.0 & US $\$ 25,728$ & US $\$ 163,205$ \\
E-105 & 1 & 11.6 & US $\$ 82,352$ & US $\$ 517,480$ \\
E-106 & 1 & 11.6 & US $\$ 26,093$ & US $\$ 163,965$ \\
F-101 & 1 & 14.0 & US $\$ 242,160$ & US $\$ 682,216$ \\
H-101 & 1 & 14.0 & US $\$ 303,140$ & US $\$ 851,823$ \\
V-101 & 2 & - & US $\$ 37,787$ & US $\$ 153,794$ \\
V-102 & 1 & 11.6 & US $\$ 1,502$ & US $\$ 11,854$ \\
V-103 & 1 & - & US $\$ 3,456$ & US $\$ 14,064$ \\
V-104 & 4 & - & US $\$ 127,432$ & US $\$ 518,649$ \\
\hline
\end{tabular}

